\title{
ALGEBRAIC LEAVES OF ALGEBRAIC FOLIATIONS OVER NUMBER FIELDS
}

\author{
by JeAN-BENôTt BOST
}

\author{
GONTENTS
}

1. Introduction . . . . . . . . . . . . . . . . . . . . . . . . . . . .

2. An algebraicity criterion for leaves of algebraic foliations over number fields . . . . . . . . . . . 166

2.1. Preliminary definitions . . . . . . . . . . . . . . . . . . . . . . . . . . . . . . . . . 166

2.1.1. Involutive bundles and foliations . . . . . . . . . . . . . . . . . . . . . . 166

2.1.2. The Liouville property for complex manifolds . . . . . . . . . . . . . . . . . . . . 167

2.2. Algebraic leaves of algebraic foliations over number fields . . . . . . . . . . . . . . . . . . . 168

2.2.1. An algebraicity criterion . . . . . . . . . . . . . . . . . . . . . . . . . . . . . 168

2.2.2. Variants . . . . . . . . . . . . . . . . . . . . . . . . . 169

2.3. Algebraic Lie algebras and restricted Lie algebras . . . . . . . . . . . . . . . . . . 171

2.3.1. Algebraic subgroups and Lie subalgebras closed under $p$-th powers . . . . . . . . . . . 171

2.3.2. Connected algebraic groups up to isogeny over number fields . . . . . . . . . . . . . 174

2.4. Principal G-bundles with flat connections . . . . . . . . . . . . . . . . . . . . . . . . . 175

2.4.1. The conjecture of Grothendieck-Katz . . . . . . . . . . . . . . . . . . . . . . . 175

2.4.2. Flat principal bundles with solvable structural groups . . . . . . . . . . . . . . . . . 177

3. Algebraization of formal germs of subvarieties in algebraic varieties over number fields . . . . . . . 182

3.1. Size of smooth formal subschemes . . . . . . . . . . . . . . . . . . . . . . . . . . 182

3.1.1. Local theory . . . . . . . . . . . . . . . . . . . . . . . . . . . . . . . . . . 182

3.1.2. Global theory . . . . . . . . . . . . . . . . . . . . . . . . . . . . . . . . 185

3.2. Main Theorem . . . . . . . . . . . . . . . . . . . . . . . . . . . . . . 185

3.3. A geometric analogue . . . . . . . . . . . . . . . . . . . . . . . . . . . . 186

3.4. Vanishing of $p$-curvatures and p-adic sizes of formal leaves . . . . . . . . . . . . . . . . . 189

3.4.1. Formal flows and formal leaves . . . . . . . . . . . . . . . . . . . . . . . . . . . 189

3.4.2. p-adic estimates on formal flows . . . . . . . . . . . . . . . . . . . . . . . . . . 191

3.4.3. Proof of Theorem 2.2 . . . . . . . . . . . . . . . . . . . . . . . . . . . . . 193

4. Proof of the main theorem . . . . . . . . . . . . . . . . . . . . . . . . . . . . . . . . 193

4.1. Hermitian vector bundles, slopes, and heights of linear maps . . . . . . . . . . . . . . . . 194

4.1.1. Basic definitions . . . . . . . . . . . . . . . . . . . . . . . . . . . . . . . . . 194

4.1.2. The maximal slope of a symmetric power . . . . . . . . . . . . . . . . . . . . . 196

4.1.3. Tensor powers of hermitian line bundles and associated hermitian vector bundles of sections 197

4.1.4. Heights of linear maps and slope inequalities . . . . . . . . . . . . . . . . . . . . . 199

4.2. Diophantine approximation using slopes and heights of evaluation maps . . . . . . . . . . . . 201

4.2.1. Notation and preliminary reductions . . . . . . . . . . . . . . . . . . . . . . . . 201

4.2.2. Slope inequalities . . . . . . . . . . . . . . . . . . . . . . . . . . . . . . . 203

4.3. Upper bounds on the heights of evaluation maps . . . . . . . . . . . . . . . . . . . . . . . 205

4.3.1. Local bounds . . . . . . . . . . . . . . . . . . . . . . . . . . . . . . . . . . 205

4.3.2. Archimedean bounds . . . . . . . . . . . . . . . . . . . . . . . . . . . . . 206

4.3.3. A variation on the First Main Theorem of value distribution theory . . . . . . . . . . 210

Appendix: smooth principal bundles and connections . . . . . . . . . . . . . . . . . . . . . . . . 215

A.1. Basic definitions . . . . . . . . . . . . . . . . . . . . . . . . . . . . . . 216

A.2. Constructions of smooth principal G-bundles and connections . . . . . . . . . . . . . . . . 217

Summary. - We prove an algebraicity criterion for leaves of algebraic foliations defined over number fields. Namely, consider a number field $\mathrm{K}$ embedded in $\mathbf{C}$, a smooth algebraic variety $\mathrm{X}$ over $\mathrm{K}$, equipped with a K-rational point $\mathrm{P}$, and $\mathrm{F}$ an algebraic subbundle of the its tangent bundle $\mathrm{T}_{\mathrm{X}}$, defined over $\mathrm{K}$. Assume moreover that the vector bundle $\mathrm{F}$ is involutive, i.e., closed under Lie bracket. Then it defines an holomorphic foliation of the analytic manifold $\mathbf{X}(\mathbf{G})$, and one may consider its leaf $\mathscr{T}$ through P. We prove that $\mathscr{F}$ is algebraic if the following local conditions are satisfied: 
i) For almost every prime ideal $\mathbf{p}$ of the ring of integers $\mathscr{O}_{\mathrm{K}}$ of the number field $\mathrm{K}$, the p-curvature of the reduction modulo $\mathbf{p}$ of the involutive bundle $\mathrm{F}$ vanishes at $\mathrm{P}$ (where $p$ denotes the characteristic of the residue field $\sigma_{\mathrm{K}} / \mathbf{p}$ ).

ii) The analytic manifold $\mathscr{F}$ satisfies the Liouville property; this arises, in particular, if $\mathscr{F}$ is the image by some holomorphic map of the complement in a complex algebraic variety of a closed analytic subset.

This algebraicity criterion unifies and extends various results of D. V. and G. V. Chudnovsky, André, and Graftieaux, and also admits new consequences. For instance, applied to an algebraic group $\mathrm{G}$ over $\mathrm{K}$, it shows that a $\mathrm{K}$-Lie subalgebra $\mathbf{h}$ of $\mathrm{Lie} \mathrm{G}$ is algebraic if and only if for almost every non-zero prime ideal $\mathbf{p}$ of $\mathscr{O}_{\mathrm{K}}$, of residue characteristic $p$, the reduction modulo $\mathbf{p}$ of $\mathbf{h}$ is a restricted Lie subalgebra of the reduction modulo $\mathbf{p}$ of Lie $\mathrm{G}$ (i.e., is stable under p-th powers). This solves a conjecture of Ekedahl and Shepherd-Barron.

The algebraicity criterion above follows from a more basic algebraicity criterion concerning smooth formal germs in algebraic varieties over number fields. The proof of the latter relies on "transcendence techniques", recast in a modern geometric version involving elementary concepts of Arakelov geometry, and on some analytic estimates, related to the First Main Theorem of higher-dimensional Nevanlinna theory.

Résumé. - Nous établissons un critère d'algébricité concernant les feuilles des feuilletages algébriques définis sur un corps de nombres. Soit en effet $\mathrm{K}$ un corps de nombres plongé dans $\mathbf{G}, \mathrm{X}$ une variété algébrique lisse sur $\mathrm{K}$, munie d'un point $\mathrm{K}$-rationnel $\mathrm{P}$, et $\mathrm{F}$ un sous-fibré du fibré tangent $\mathrm{T}_{\mathrm{X}}$, défini sur $\mathrm{K}$. Supposons de plus que le fibré vectoriel $\mathrm{F}$ soit involutif, i.e., stable par crochet de Lie. Il définit alors un feuilletage holomorphe de la variété analytique $\mathrm{X}(\mathbf{G})$ et l'on peut considérer la feuille $\mathscr{T}$ de ce feuilletage passant par $\mathrm{P}$. Nous montrons que $\mathscr{F}$ est algébrique lorque les conditions locales suivantes sont satisfaites:

i) Pour presque tout idéal premier $\mathbf{p}$ de l'anneau des entiers $\mathscr{O}_{\mathrm{K}}$ de $\mathrm{K}$, la réduction modulo $\mathbf{p}$ du fibré $\mathrm{F}$ est stable par l'opération de puissance p-ième (où $p$ désigne la caractéristique du corps résiduel $\mathscr{O}_{\mathrm{K}} / \mathbf{p}$ ).

ii) La variété analytique $\mathscr{T}$ satisfait à la propriété de Liouville; cela a lieu, par exemple, lorsque $\mathscr{T}$ est l'image par une application holomorphe du complémentaire d'un sous-ensemble analytique fermé dans une variété algébrique.

Ce critère d'algébricité unifie et généralise divers résultats de D. V. and G. V. Chudnovsky, André et Graftieaux. Il conduit aussi à de nouvelles conséquences. Par exemple, appliqué à un groupe algébrique $G$ $\operatorname{sur} \mathrm{K}$, il montre qu'une sous-algèbre de Lie $\mathbf{h}$ de Lie $\mathrm{G}$, définie sur $\mathrm{K}$, est algébrique si et seulement si, pour presque tout idéal premier $\mathbf{p}$ de $\mathscr{O}_{\mathrm{K}}$, de caractéristique résiduelle $p$, la réduction modulo $\mathbf{p}$ de $\mathbf{h}$ est une sous-p-algèbre de Lie de la réduction modulo $\mathbf{p}$ de $\mathrm{Lie}_{\mathrm{G}}$ (i.e., est stable par puissance p-ième). Cet énoncé résout une conjecture d'Ekedahl et Shepherd-Barron.

Le critère d'algébricité ci-dessus découle d'un critère d'algébricité plus général, concernant les germes de sous-variétés formelles des variétés algébriques sur les corps de nombres. La démonstration de ce dernier repose sur des "techniques de transcendance", reformulées dans une version géométrique utilisant diverses notions élémentaires de géométrie d'Arakelov, et sur des estimations analytiques reliées au premier théorème fondamental de la théorie de Nevanlinna en dimension supérieure.

Conventions. - The following notation and terminology are used throughout this paper.

The rank of a vector bundle $\mathrm{E}$ (resp. of a linear map $\varphi$ ) will be denoted $\mathrm{rk} \mathrm{E}$ (resp. $\mathrm{rk} \varphi)$.

By an algebraic variety over some field $k$, we mean an integral separated scheme of finite type over $k$. Integral subschemes of such an algebraic variety $\mathrm{X}$ over $k$ will be called algebraic subvarieties of $\mathrm{X}$.

If $p$ is a prime number and $\mathrm{X}$ a scheme over the finite field with $p$ elements $\mathbf{F}_{p}$, we will denote by Frob $: \mathrm{X} \rightarrow \mathrm{X}$ the absolute Frobenius morphism of $\mathrm{X}$, defined by the endomorphism $a \mapsto a^{p}$ of the sheaf of rings $\mathscr{C}_{\mathrm{X}}$.

If $\mathrm{K}$ is a number field, its ring of integers will be denoted $\mathscr{O}_{\mathrm{K}}$ and the set of its finite places (or, equivalently, the set of non-zero prime ideals of $\mathscr{C}_{\mathrm{K}}$, or of closed points of Spec $\mathscr{O}_{\mathrm{K}}$ ) will be denoted $\mathrm{V}_{f}(\mathrm{~K})$. For any $\mathbf{p}$ in $\mathrm{V}_{f}(\mathrm{~K})$, we let $\mathbf{F}_{\mathbf{p}}$ be the finite field $\mathscr{O}_{\mathrm{K}} / \mathbf{p}, \mathrm{Np}:=\left|\mathbf{F}_{\mathbf{p}}\right|$ the norm of $\mathbf{p}, \mathrm{K}_{\mathbf{p}}\left(\right.$ resp. $\left.\mathscr{O}_{\mathbf{p}}\right)$ the $\mathbf{p}$-adic completion of $\mathrm{K}$ (resp. of $\left.\mathscr{C}_{\mathrm{K}}\right)$, and ||$_{\mathbf{p}}$ the $\mathbf{p}$-adic absolute value on $\mathrm{K}_{\mathbf{p}}$ normalized in such a way that, for any uniformizing element $\varpi$ in $\mathscr{O}_{\mathbf{p}}$, we have:

$$
|\bowtie|_{\mathbf{p}}=\mathrm{Np}^{-1} ;
$$


equivalently, if $p$ denotes the residue characteristic of $\mathbf{p}$ and $e$ the absolute ramification index of $\mathrm{K}_{\mathrm{p}}$, then:

$$
|p|_{\mathbf{p}}=\mathrm{Np}^{-e}=p^{-\left[\mathrm{K}_{\mathbf{p}}: \mathbf{Q}_{p}\right]}
$$

If $\Lambda$ is an $\mathscr{O}_{\mathbf{p}}$-lattice in some finite dimensional $\mathrm{K}_{\mathbf{p}}$-vector space $\mathrm{E}$, the $\mathbf{p}$-adic norm \|\| on $\mathrm{E}$ attached to $\Lambda$ is defined by the equality

$$
\left\|\sum_{i=1}^{n} x_{i} e_{i}\right\|:=\max _{1 \leqslant i \leqslant n}\left|x_{i}\right|_{\mathbf{p}},
$$

for any $\mathcal{O}_{\mathbf{p}}$-basis $\left(e_{1}, \ldots, e_{n}\right)$ of $\Lambda$ and any $\left(x_{1}, \ldots, x_{n}\right) \in \mathrm{K}^{n}$.

On a complex analytic manifold, we write as usual $d=\partial+\bar{\partial}$ and we let

$$
d^{c}:=(i / 4 \pi)(\bar{\partial}-\partial) ;
$$

consequently:

$$
d d^{c}=(i / 2 \pi) \partial \bar{\partial}
$$

\section{Introduction}

In this paper, we prove an algebraicity criterion for leaves of algebraic foliations defined over a number field. Namely, consider a number field $\mathrm{K}$ embedded in $\mathbf{C}$, a smooth algebraic variety $\mathrm{X}$ over $\mathrm{K}$, equipped with a $\mathrm{K}$-rational point $\mathrm{P}$, and $\mathrm{F}$ an algebraic sub-vector bundle of the tangent bundle $\mathrm{T}_{\mathrm{X}}$, defined over $\mathrm{K}$. Assume moreover that the vector bundle $\mathrm{F}$ is involutive, i.e., closed under Lie bracket. Then it defines an holomorphic foliation of the analytic manifold $\mathrm{X}(\mathbf{G})$, and one may wonder if the leaf $\mathscr{F}$ through $\mathrm{P}$ of this foliation is an algebraic subvariety of $\mathrm{X}$. (Recall that, in general, $\mathscr{F}$ is a connected analytic manifold of dimension the rank of $\mathrm{F}$, and that the inclusion map $\mathscr{F} \hookrightarrow \mathrm{X}(\mathbf{G})$ is an injective holomorphic immersion, the image of which may possibly be dense in the analytic topology.) We prove that $\mathscr{F}$ is algebraic if the following local conditions are satisfied:

1. For almost every prime ideal $\mathbf{p}$ of the ring of integers $\mathscr{O}_{\mathrm{K}}$ of the number field $\mathrm{K}$, the reduction modulo $\mathbf{p}$ of the involutive bundle $\mathrm{F}$ is stable by $p$-th power, where $p$ denotes the characteristic of $\mathbf{F}_{\mathbf{p}}$.

2. The analytic manifold $\mathscr{F}$ satisfies the Liouville property (see infra 2.1.2); this condition is satisfied for instance when $\mathscr{F}$ is the image by some holomorphic map of the complement in a complex algebraic variety of a closed analytic subset.

Applied to translation invariant foliations on an algebraic group $\mathrm{G}$ over $\mathrm{K}$, this criterion shows that a Lie subalgebra $\mathbf{h}$ of Lie $\mathrm{G}$ (defined over $\mathrm{K}$ ) is algebraic if and only if for almost every non-zero prime ideal $\mathbf{p}$ of $\mathscr{C}_{\mathrm{K}}$, of residue characteristic $p$, the reduction modulo $\mathbf{p}$ of $\mathbf{h}$ is a restricted Lie subalgebra of the reduction modulo $\mathbf{p}$ of Lie $\mathrm{G}$ (i.e., is stable under p-th powers; see Theorem 2.3 below). The equivalence of these two conditions may be seen as an 
analogue, for algebraic groups, of the famous conjecture of Grothendieck-Katz relating the $p$-curvatures of algebraic linear differential equations to their differential Galois groups (cf. [Kat72] and [Kat82]; see also [And99] for recent results and comprehensive references).

The present work has been motivated by the papers [CC85a] and [CC85b] of D. V. and G. V. Chudnovsky, and by their more recent developments due to André ([And89]; see also [And99]) and Graftieaux ([Gr98] and [Gr01a]). A simple but crucial innovation in our approach is the introduction of foliations, which leads to a general theorem unifying various previous results about either systems of linear differential equations, or particular commutative algebraic groups.

Actually, our algebraicity criterion for foliations follows from a more basic algebraicity criterion concerning smooth formal germs of subvarieties inside an algebraic variety over a number field ( $c f$. Theorem 3.4, infra). This criterion relies on some notion of size, defined for formal germs inside algebraic varieties over $p$ adic fields: it involves the collection of sizes associated to such a formal germ over a number field $\mathrm{K}$ by considering its various completions, in a way reminiscent of the so-called Bombieri conditions in the theory of G-functions. This algebraicity criterion may also be seen as an arithmetic counterpart of some geometric algebraicity criteria (see Theorems 3.5 and 3.6 infra), closely related to the classical work of Hironaka, Matsumura, and Hartshorne on formal functions ([Hir68], [HM68], [Har68]).

Our proofs of algebraicity criteria rely on "transcendence techniques", inspired by the ones in the above mentioned works, but of a rather unconventional nature. Indeed, we avoid the traditional constructions of "auxiliary polynomials" by using instead a few basic concepts from Arakelov geometry - namely, the formalism of slopes for hermitian vector bundles over arithmetic curves - which provide a concise and geometric reformulation of basic transcendence and diophantine approximation techniques. (This new approach was introduced in [Bos96] in relation with the work of Masser and Wüstholz on abelian varieties, but has a much wider scope; see for instance [Gr01a], [Gr01b], and [CB01].)

The basic idea of our algebraicity proofs may be roughly described as follows. Let $\mathrm{X}$ be a projective variety over a number field $\mathrm{K}$, and $\mathrm{L}$ an ample line bundle on $\mathrm{X}$. To prove that a (say smooth) formal subscheme $\hat{\mathrm{V}}$ of the completion $\hat{\mathrm{X}}_{\mathrm{P}}$ of $\mathrm{X}$ at a rational point $\mathrm{P}$ in $\mathrm{X}(\mathrm{K})$ - supposed to satisfy suitable local conditions - is a branch of some algebraic subvariety $\mathrm{Y}$ of $\mathrm{X}$ through $\mathrm{P}$, we consider the successive infinitesimal neighbourhoods of $\mathrm{P}$ in $\hat{\mathrm{V}}$ :

$$
\mathrm{V}_{0}=\{\mathrm{P}\} \subset \mathrm{V}_{1} \subset \ldots \subset \mathrm{V}_{n} \subset \mathrm{V}_{n+1} \subset \ldots
$$

and, for any $(\mathbf{D}, n) \in \mathbf{N} \times \mathbf{N}$, we introduce the evaluation map

$$
\varphi_{\mathrm{D}, n}: \Gamma\left(\mathrm{X}, \mathrm{L}^{\otimes \mathrm{D}}\right) \longrightarrow \mathrm{L}_{\mid \mathrm{V}_{n}}^{\otimes \mathrm{D}} .
$$


Basically, we need to show that, for any large $\mathrm{D}$, the kernel of $\varphi_{\mathrm{D}, n}$ stays large enough when $n$ goes to infinity. To achieve this, we choose models over the ring of integers $\mathscr{C}_{\mathrm{K}}$, as well as hermitian structures at infinite places, for $\mathrm{X}, \mathrm{L}$, and $\mathrm{T}_{\hat{\mathrm{V}}}, \mathrm{P}$. From these data, we deduce integral and hermitian structures on $\Gamma\left(\mathrm{X}, \mathrm{L}^{\otimes \mathrm{D}}\right)$ and (subquotients of) $\mathrm{L}_{\mid \mathrm{V}_{n}}^{\otimes \mathrm{D}}$, which therefore possess Arakelov type invariants, their Arakelov degrees and more generally, their Arakelov slopes; these structures also allow to attach a natural height to $\varphi_{\mathrm{D}, n}$. The local conditions on $\hat{\mathrm{V}}$ are used to show that these heights are "very negative" - or, roughly speaking, that $\varphi_{\mathrm{D}, n}$ is "very small" - when $n / D$ goes to infinity. This would contradict the asymptotic behaviour of the slopes of $\Gamma\left(\mathrm{X}, \mathrm{L}^{\otimes \mathrm{D}}\right)$ and $\mathrm{L}_{\mid \mathrm{V}_{n}}^{\otimes \mathrm{D}}$ if the kernel of $\varphi_{\mathrm{D}, n}$ were too small.

A crucial point in this line of arguments is the "smallness" of $\varphi_{\mathrm{D}, n}$ at some archimedean place, which will be derived from the Liouville property by means of analytic techniques closely related to the First Main Theorem of higher dimensional Nevanlinna theory.

A short time after the results in this paper had been announced, N. ShepherdBarron kindly sent me a copy of his joint work [ESB99] with T. Ekedahl, where they present some evidence for what they call Conjecture $F$, namely, that an involutive bundle $\mathrm{F}$ as above is algebraically integrable if (and only if) for almost every prime ideal $\mathbf{p}$ of $\mathscr{O}_{\mathrm{K}}$, the p-curvature of the reduction modulo $\mathbf{p}$ of the bundle $\mathrm{F}$ vanishes identically. Most of this evidence concerns the special case of the conjecture where the bundle $\mathrm{F}$ is a translation invariant bundle over an abelian variety, which they state as Conjecture $A$, and is now solved by Theorem 2.3 below.

Finally, I would like to mention the recent papers of Bogomolov and McQuillan [BMQ01] and Chambert-Loir [CB01], which present very interesting developments related to the results of this paper.

This paper is organized as follows.

Firstly, in Section 2, we state our algebraicity criterion for leaves of algebraic foliations, and we derive various applications of it, where, in some way or another, it is possible to get rid of the Liouville condition. Besides translation invariant foliations on algebraic groups mentioned above, we apply it to foliations defined by flat connections on principal bundles, and we recover and extend earlier results, due to D. V. and G. V. Chudnovsky and André, concerning the original Grothendieck-Katz conjecture.

Section 3 is devoted to the formulation of the algebraicity criterion concerning smooth formal germs in algebraic varieties over number fields from which our result on foliations is derived. In particular, we introduce and discuss the basic properties of the relevant notion of "size" for formal germs, and we discuss the geometric counterpart of this second algebraicity criterion and its relation with classical results such as Hartshorne's G2 Theorem. We also show that it indeed entails the algebraicity 
criterion for leaves of foliations discussed in Section 2 by establishing lower bounds for the $p$-adic sizes of germs of formal leaves of foliations.

Finally, in Section 4, we establish the algebraicity criterion for formal germs, along the lines described above. The fundamental simplicity of this proof is worth mentioning: besides some basic notions in algebraic geometry, it only relies on some rudimentary concepts of Arakelov geometry (the definitions of which use only the product formula for number fields; $c f .4$.1, infra), some elementary estimates concerning power series with coefficients in $p$-adic fields and their radii of convergence, and simple properties of plurisubharmonic functions on complex manifolds.

It is a pleasure to thank P. Gille, L. Illusie, Y. Laszlo, and M. Raynaud for helpful questions and advice during the writing of this paper, and A. Ancona and N. Sibony for illuminating discussions concerning potential theory and complex manifolds. I am also very grateful to Y. André and P. Graftieaux for the communication of their papers [And99] and [Gr01a] and related discussions, and to A. Chambert-Loir, M. McQuillan, J.-P. Serre, and the referees for comments and suggestions on the first version of this paper.

A first version of our main algebraization theorem (Theorem 3.4, infra), based on the techniques in Section 4.3.3 below, has been presented during the conference Alan Baker 60 - Number Theory and Diophantine Geometry held at E.T.H., Zürich, in September 1999; I am very grateful to G. Wüstholz, who invited me to talk at this conference, and so gave me the opportunity to clarify the application of the "method of slopes" to algebraicity results "à la Chudnovsky". The consequences of Theorem 3.4 concerning foliations and algebraic groups (in particular Theorems 2.1 and 2.3, infra) have been announced during a talk at the Séminaire d'arithmétique et de géométrie algébrique of Orsay, in January 2000.

\section{An algebraicity criterion for leaves of algebraic foliations over number fields}

\subsection{Preliminary definitions}

\subsubsection{Involutive bundles and foliations}

Let $\mathrm{X}$ be a smooth algebraic variety over some field $\mathrm{K}$, and let $\mathrm{F}$ be a subvector bundle of the tangent bundle $\mathrm{T}_{\mathrm{X}}$ of $\mathrm{X}$ (defined over $\mathrm{K}$ ). Recall that $\mathrm{F}$ is said to be involutive if its sheaf of regular sections is closed under Lie bracket, and that a smooth algebraic subvariety $\mathrm{Y}$ of $\mathrm{X}$ is called an integral subvariety of $\mathrm{F}$ if the differential $D i: \mathrm{T}_{\mathrm{Y}} \rightarrow i^{*} \mathrm{~T}_{\mathrm{X}}$ of the inclusion map $i: \mathrm{Y} \rightarrow \mathrm{X}$ maps $\mathrm{T}_{\mathrm{Y}}$ to $i^{*} \mathrm{~F}$. These definitions are clearly geometric, i.e., invariant under extension of the base field $\mathrm{K}$. 
When the characteristic $p$ of $\mathrm{K}$ is positive, the sheaf of regular sections of $\mathrm{T}_{\mathrm{X}}$, which may be identified with the sheaf of derivations $\operatorname{Der}_{\mathrm{K}}\left(\mathscr{O}_{\mathrm{X}}\right)$, is endowed with the $p$-th power operation, which maps any local $\mathrm{K}$-derivation $\mathrm{D}$ of $\mathscr{O}_{\mathrm{x}}$ to its $p$-th iterate $\mathrm{D}^{[\beta]}$. When $\mathrm{F}$ is involutive, the map which sends $\mathrm{D}$ to the class in $\mathrm{T}_{\mathrm{X}} / \mathrm{F}$ of $\mathrm{D}^{[\beta]}$ is $p$-linear and defines a section of $F r o b * \check{\mathrm{F}} \otimes \mathrm{T}_{\mathrm{X}} / \mathrm{F}$ over $\mathrm{X}$, the $p$-curvature of $\mathrm{F}$. The subbundle $\mathrm{F}$ is said to be closed under $p$-th powers if, for any regular local section $\mathrm{D}$ of $\mathrm{F}$, the section $\mathrm{D}^{[p]}$ of $\mathrm{T}_{\mathrm{X}}$ is indeed a section of $\mathrm{F}$, or equivalently, when $\mathrm{F}$ is involutive, if its $p$-curvature vanishes everywhere. Also observe that the $p$-curvature of an involutive subbundle $\mathrm{F}$ of $\mathrm{T}_{\mathrm{X}}$ vanishes along any smooth integral subvariety of $\mathrm{F}$. (See [Eke87], [Miy87], and [MP97], Part I, Lecture III, for more information concerning involutive bundles and foliations in characteristic $p>0$.)

When $\mathrm{K}$ is a subfield of $\mathbf{C}$, the vector bundle $\mathbf{F}$ defines a holomorphic subbundle $\mathrm{F}_{\mathrm{G}}$ of the holomorphic tangent bundle of the complex manifold $\mathrm{X}(\mathbf{G})$. If $\mathrm{F}$ is involutive, then $\mathrm{F}_{\mathbf{G}}$ is analytically involutive and defines a holomorphic foliation of $\mathrm{X}(\mathbf{G})$; moreover, for any $\mathrm{P} \in \mathrm{X}(\mathrm{K})$, the following conditions are easily seen to be equivalent:

1. There exists an algebraic subset of dimension $r:=r k \mathrm{~F}$ of $\mathrm{X}(\mathbf{C})$ containing the germ of leaf of $\mathrm{F}_{\mathrm{G}}$ through $\mathrm{P}$.

2. The maximal leaf $\mathscr{F}$ of $\mathrm{F}_{\mathbf{G}}$ through $\mathrm{P}$ in $\mathrm{X}(\mathbf{G})$ is a closed algebraic subvariety of $\mathrm{X}(\mathbf{G})$ (necessarily smooth).

3. There exists a (necessarily unique) smooth closed connected algebraic Ksubvariety $\mathrm{Y}$ in $\mathrm{X}$ of dimension $r$, which contains $\mathrm{P}$ and is an integral subvariety of $\mathrm{F}$.

When they are satisfied, the maximal leaf $\mathscr{F}$ coincides with the set of complex points $\mathrm{Y}(\mathbf{G})$ of $\mathrm{Y}$ and we shall say that the leaf of $\mathrm{F}$ through $\mathrm{P}$ is algebraic.

\subsubsection{The Liouville property for complex manifolds}

We shall say that a connected complex manifold M satisfies the Liouville property when every plurisubharmonic function on $\mathrm{M}$ bounded from above is constant, or, equivalently, when every bounded plurisubharmonic function on $\mathrm{M}$ is constant (see for instance [Hör94], chapter IV, or [Kli91], chapter 2, for basic properties of plurisubharmonic functions).

Examples of complex manifolds satisfying the Liouville property are provided by the following simple observations:

L1. The affine space $\mathbf{M}=\mathbf{G}^{\mathrm{N}}$ satisfies the Liouville property. This is well known when $\mathrm{N}=1$, and this special case entails the general one.

L2. Any connected compact complex manifold $\mathrm{M}$ satisfies the Liouville property. In fact, any plurisubharmonic function on $\mathrm{M}$ is constant by the maximum modulus principle.

L3. If a connected complex manifold $\mathrm{M}$ satisfies the Liouville property and if $\mathrm{F}$ is a closed pluripolar subset (e.g., a closed analytic subset of positive codimension) of $\mathrm{M}$, then $\mathrm{M} \backslash \mathrm{F}$ satisfies the 
Liouville property. Indeed, any plurisubharmonic function bounded from above on $\mathrm{M} \backslash \mathrm{F}$ extends to a plurisubharmonic function on $\mathrm{M}$.

L4. Let $\mathbf{M}$ and $\mathbf{M}^{\prime}$ be two connected complex manifolds and $f: \mathbf{M}^{\prime} \rightarrow \mathbf{M}$ a holomorphic map. If $f\left(\mathbf{M}^{\prime}\right)$ contains an open subset of $\mathbf{M}$, the complement of which is a Lebesgue null set, and if $\mathrm{M}^{\prime}$ satisfies the Liouville property then so does $\mathrm{M}$. This follows from the fact that for any plurisubharmonic function $\varphi$ on $\mathrm{M}$, the function $f^{*} \varphi:=\varphi \circ f$ is plurisubharmonic on $\mathbf{M}^{\prime}$. Conversely, when $\mathbf{M}$ and $\mathbf{M}^{\prime}$ have the same dimension and $f$ is surjective and proper, if $\mathbf{M}$ satisfies the Liouville property then so does $\mathrm{M}^{\prime}$. To prove this, observe that, by Property $\mathbf{L} 3$ above, we may assume that $f: \mathbf{M}^{\prime} \rightarrow \mathrm{M}$ is a finite étale covering; then, if $\varphi: \mathbf{M}^{\prime} \rightarrow[0,1]$ is plurisubharmonic and if $p \in\left[1,+\infty\left[\right.\right.$, the non-negative function $\psi_{p}$ on $\mathrm{M}$ defined by $\psi_{p}(x):=\left(\sum_{x^{\prime} \in f^{-1}(x)} \varphi\left(x^{\prime}\right)^{p}\right)^{1 / p}$ is plurisubharmonic and bounded by the degree of $f$, hence constant; this shows that $\varphi\left(f^{-1}(x)\right)$ does not depend on $x$, and consequently that $\varphi$ takes a finite number of values and therefore is constant.

From L1-4, it follows that the manifold of complex points of any smooth connected complex algebraic A variety satisfies the Liouville property. One may indeed assume that A is affine, and observe, either that it may be realized as the complement of a divisor in some projective smooth complex connected variety (by Hironaka's resolution of singularities), or that there exists some finite morphism from $\mathbf{A}$ onto the affine space $\mathbf{A}_{\mathbf{C}}^{\text {dimM }}$.

Observe however that complex manifolds satisfying the Liouville property are far from exhausted by smooth complex algebraic varieties and complements of their closed analytic or pluripolar subsets. For instance, any connected complex Lie group satisfies it. Indeed, if $\mathrm{G}$ is such a group and if $\mathrm{E} \subset \mathrm{G}$ is the image of the exponential map from Lie $G$ to $G$, then $\mathbf{L} 1$ shows that any bounded plurisubharmonic function $\varphi$ on $\mathrm{G}$ is constant on each translate $g . \mathrm{E}, g \in \mathrm{G}$, hence locally constant on G. For further examples, see for instance Proposition 2.10, infra, and [Tak93] (where a criterion for the Liouville property involving volume growth is established).

In complex dimension 1, manifolds satisfying the Liouville property are the Riemann surfaces which, equipped with any smooth conformal metric, are recurrent Riemannian varieties, i.e., Riemannian varieties whose Brownian motion is a recurrent process (see for instance [Anc90] or [Gri99]). These Riemann surfaces are classically said to be parabolic, or to belong to the class $\mathrm{O}_{\mathrm{G}}$ (see for instance [AS60], Chapter IV).

2.2. Algebraic leaves of algebraic foliations over number fields

\subsubsection{An algebraicity criterion}

Theorem 2.1. - Let $\mathrm{X}$ be a smooth algebraic variety over a number field $\mathrm{K}, \mathrm{F}$ an involutive subbundle of the tangent bundle $\mathrm{T}_{\mathrm{X}}$ of $\mathrm{X}$ (defined over $\mathrm{K}$ ), and $\mathrm{P}$ a point in $\mathrm{X}(\mathrm{K})$. For some sufficiently divisible integer $\mathrm{N}$, let $\mathscr{C}($ resp. $\mathscr{F})$ be a smooth model of $\mathrm{X}$ over $\mathrm{S}:=\operatorname{Spec} \mathscr{O}_{\mathrm{K}}[1 / \mathrm{N}]$ (resp. a sub-vector bundle of the relative tangent bundle $\mathrm{T}_{\mathscr{X}} / \mathrm{s}$ over $\mathscr{\mathscr { C }}$ such that $\mathscr{F}_{\mathrm{K}}$ coincides with F). 
Assume that the following two conditions are satisfied:

i) For almost every non-zero prime ideal $\mathbf{p}$ of $\mathscr{O}_{\mathrm{K}}[1 / \mathrm{N}]$, the subbundle $\mathscr{F}_{\mathbf{F}_{\mathbf{p}}}$ of $\mathrm{T}_{\mathscr{X} / \mathrm{S}, \mathbf{F}_{\mathbf{p}}} \simeq \mathrm{T}_{\mathscr{X}}{ }_{\mathbf{F}_{\mathbf{p}}}$ over $\mathscr{C}_{\mathbf{F}_{\mathbf{p}}}$ is stable by $p$-th power, where p-denotes the characteristic of $\mathbf{F}_{\mathbf{p}}$.

ii) There exists an embedding $\sigma_{0}: \mathbf{K} \hookrightarrow \mathbf{C}$ such that the analytic leaf through $\mathrm{P}_{\sigma_{0}}$ of the involutive holomorphic bundle $\mathrm{F}_{\sigma_{0}}$ on the complex analytic manifold $\mathrm{X}_{\sigma_{0}}(\mathbf{G})$ contains an open neighbourhood of $\mathrm{P}_{\sigma_{0}}$ which satisfies the Liouville property.

Then the leaf of $\mathrm{F}$ through $\mathrm{P}$ is algebraic.

Observe that condition ii) is implied by the following one:

ii)' There exist an embedding $\boldsymbol{\sigma}_{0}: \mathrm{K} \hookrightarrow \mathbf{C}$, a complex manifold $\mathrm{M}$ satisfying the Liouville property, a point $\mathrm{O}$ of $\mathrm{M}$, and a holomorphic map $f: \mathrm{M} \rightarrow \mathrm{X}_{\sigma_{0}}(\mathbf{G})$, which sends $\mathrm{O}$ to $\mathrm{P}$ and maps the germ of $\mathrm{M}$ at $\mathrm{O}$ biholomorphically onto the germ of leaf through $\mathrm{P}$ of the involutive holomorphic bundle $\mathrm{F}_{\sigma_{0}}$ on $\mathrm{X}_{\sigma_{0}}(\mathbf{G})$.

Indeed, it follows from Section 2.1.2. that the open submanifold of $\mathrm{M}$ where $f$ is étale satisfies the Liouville property, and also its image, which is an open neighbourhood of $\mathrm{P}_{\sigma_{0}}$ in the leaf of $\mathrm{P}_{\sigma_{0}}$ through $\mathrm{F}_{\sigma_{0}}$.

In turn, condition ii) ${ }^{\prime}$ is implied by:

ii)" There exist an embedding $\sigma_{0}: \mathrm{K} \hookrightarrow \mathbf{C}$, a complex algebraic variety $\mathrm{A}$, a smooth point $\mathrm{O}$ of $\mathrm{A}(\mathbf{G})$, and a meromorphic map $f: \mathrm{A}(\mathbf{G}) \rightarrow \mathrm{X}_{\sigma_{0}}(\mathbf{G})$, holomorphic on some neighbourhood of $\mathrm{O}$, which maps the germ of $\mathrm{A}(\mathbf{C})$ at $\mathrm{O}$ biholomorphically onto the germ of leaf through $\mathrm{P}_{\sigma_{0}}$ of the involutive holomorphic bundle $\mathrm{F}_{\sigma_{0}}$ on $\mathrm{X}_{\sigma_{0}}(\mathbf{G})$.

Indeed, the maximal open subset of the complex manifold of smooth points of $\mathbf{A}(\mathbf{G})$ on which $f$ is holomorphic satisfies the Liouville property.

Conditions i) and ii) for the algebraicity of the leaf of $\mathrm{F}$ through $\mathrm{P}$ are "almost necessary". Indeed, the algebraicity of this leaf obviously implies ii)", hence ii). It also implies that, for almost every non-zero prime ideal $\mathbf{p}$ of $\mathscr{O}_{\mathrm{K}}[1 / \mathrm{N}]$, the $p$-curvature of the subbundle $\mathscr{F}_{\mathbf{F}_{\mathrm{p}}}$ of $\mathrm{T}_{\mathscr{X}_{\mathbf{F}_{\mathbf{p}}}}$ over $\mathscr{C}_{\mathbf{F}_{\mathrm{p}}}$ vanishes at $\mathrm{P}_{\mathbf{F}_{\mathrm{p}}}$, and the algebraic integrability (cf. 2.2.2. infra) of the involutive vector bundle $\mathrm{F}$ implies $\mathrm{i}$ ).

Their sufficiency will be established in Section 3.4, as a consequence of the main technical result in this paper, namely Theorem 3.4.

Theorem 2.1 should be compared with a famous theorem of Miyaoka concerning the algebraicity of leaves of an involutive vector bundle on a complex projective variety ([Miy87]; see also the simpler approaches of Shepherd-Baron, in [SB92] and [MP97], and of Bogomolov and McQuillan in [BMQ01]): Miyaoka's result holds under a purely geometric hypothesis of positivity on $\mathrm{F}$, which is never satisfied in the arithmetic applications of Theorem 2.1 which we develop in the next subsections.

\subsubsection{Variants}

In this section, I would like to mention briefly some variants of Theorem 2.1. Firstly, it is possible to weaken the regularity hypothesis on the variety $\mathrm{X}$, in the 
spirit of [BMQ01]: X may be assumed, say, only normal provided the foliation is still smooth, i.e., may be described locally (in the analytic topology) as a relatively smooth analytic fibration.

Secondly, it is possible to prove a version of Theorem 2.1 concerning the algebraicity, not of a single leaf, but of all leaves of the involutive bundle $\mathrm{F}$ simultaneously.

Consider indeed a smooth variety $\mathrm{X}$ over a field $\mathrm{K}$ of characteristic 0 , equipped with an involutive subbundle $\mathrm{F}$ of $\mathrm{T}_{\mathrm{X}}$. We may introduce the formal graph of the foliation $(\mathrm{X}, \mathrm{F})$, namely the smooth formal subscheme of the completion of $\mathrm{X} \times_{\mathrm{K}} \mathrm{X}$ along the diagonal $\Delta_{\mathrm{X}}$, admitting $\Delta_{\mathrm{X}}$ as subscheme of definition, such that:

(i) $\mathrm{G}^{\text {for }}(\mathrm{X}, \mathrm{F})$ is a formal equivalence relation on $\mathrm{X}$;

(ii) the normal bundle $\mathrm{N}_{\Delta_{\mathrm{X}}} \mathrm{G}^{\text {for }}(\mathrm{X}, \mathrm{F})$ of $\mathrm{G}^{\text {for }}(\mathrm{X}, \mathrm{F})$ along $\Delta_{\mathrm{X}}$ coincides with the subbundle $\mathrm{F}$ of $\mathrm{N}_{\Delta_{\mathrm{X}}}\left(\mathrm{X} \times_{\mathrm{K}} \mathrm{X}\right) \simeq \mathrm{T}_{\mathrm{X}}$.

When $\mathrm{K}=\mathbf{C}$, the formal graph $\mathrm{G}^{\text {for }}(\mathrm{X}, \mathrm{F})$ is the formal subscheme defined by a germ of analytic submanifold $\mathrm{G}^{a n}(\mathrm{X}, \mathrm{F})$ of $\mathrm{X}(\mathbf{G}) \times \mathrm{X}(\mathbf{G})$ along $\Delta_{\mathrm{X}(\mathbf{C})}$. Moreover, for any $\mathrm{P} \in \mathrm{X}(\mathbf{G})$, the intersection of $\mathrm{G}^{a n}(\mathrm{X}, \mathrm{F})$ with $\mathrm{X}(\mathbf{G}) \times\{\mathrm{P}\} \simeq \mathrm{X}(\mathbf{G})$ coincides with the analytic germ of leaf through $\mathrm{P}$ of the analytic foliation on $\mathrm{X}(\mathbf{G})$ defined by $\mathrm{F}$.

We shall say that the involutive bundle $\mathrm{F}$ is algebraically integrable when the Zariski closure $^{(1)}$ of $\mathrm{G}^{\text {for }}(\mathrm{X}, \mathrm{F})$ in $\mathrm{X} \times_{\mathrm{K}} \mathrm{X}$ has the same dimension $\operatorname{dim} \mathrm{X}+\mathrm{rk} \mathrm{F}$ as $\mathrm{G}^{\text {for }}(\mathrm{X}, \mathrm{F})$. This is a geometric condition (i.e., invariant by extension of the base field $\mathrm{K}$ ). When $\mathrm{K}=\mathbf{C}$, this Zariski closure coincides with the one of $\mathrm{G}^{\text {an }}(\mathrm{X}, \mathrm{F})$, and the algebraic integrability of $\mathrm{F}$ is easily seen to imply the algebraicity of all the leaves of the analytic foliation of $\mathrm{X}(\mathbf{C})$ defined by $\mathrm{F}$ - indeed, the converse application also holds (use Baire's theorem to prove that if all the leaves of this analytic foliation are algebraic, then they form a bounded family; compare [ESB99], §2).

Theorem 2.2. - Let $\mathrm{X}$ be a smooth geometrically connected algebraic variety over a number field $\mathrm{K}$ and $\mathrm{F}$ an involutive subbundle of the tangent bundle $\mathrm{T}_{\mathrm{X}}$ of $\mathrm{X}$ (defined over $\mathrm{K}$ ). For some sufficiently divisible integer $\mathrm{N}$, let $\mathscr{C}$ (resp. $\mathscr{F})$ be a smooth model of $\mathrm{X}$ over $\mathrm{S}:=\operatorname{Spec} \mathscr{O}_{\mathrm{K}}[1 / \mathrm{N}]$ (resp. a sub-vector bundle of the relative tangent bundle $\mathrm{T}_{\mathscr{X}} / \mathrm{s}$ over $\mathscr{C}$ such that $\mathscr{F}_{\mathrm{K}}$ coincides with $\mathrm{F}$ ).

Then the involutive bundle $\mathrm{F}$ is algebraically integrable if and only if the following two conditions are satisfied:

i) For almost every non-zero prime ideal $\mathbf{p}$ of $\mathscr{O}_{\mathrm{K}}[1 / \mathrm{N}]$, the reduction modulo $\mathbf{p}$ of $\mathrm{F}$ is stable by p-th power (where p-denotes the characteristic of $\mathbf{F}_{\mathbf{p}}$ ).

ii) There exist a complex manifold $\mathrm{M}$ satisfying the Liouville property, an embedding $\sigma_{0}: \mathbf{K} \hookrightarrow \mathbf{G}$, a holomorphic embedding $i: \mathbf{X}_{\sigma_{0}}(\mathbf{G}) \rightarrow \mathrm{M}$ and a holomorphic map $f:$ $\mathrm{M} \rightarrow \mathrm{X}_{\sigma_{0}}(\mathbf{G}) \times \mathrm{X}_{\sigma_{0}}(\mathbf{G})$ such that $f \circ i$ coincide with the diagonal embedding $\Delta_{\mathbf{X}_{\sigma_{0}}}(\mathbf{C})$ :

(1) Namely, the smallest closed subscheme of $\mathrm{X} \times_{\mathrm{K}} \mathrm{X}$ containing $\mathrm{G}^{\text {for }}(\mathrm{X}, \mathrm{F})$; one easily checks that it is reduced. 
$\mathrm{X}_{\sigma_{0}}(\mathbf{G}) \hookrightarrow \mathrm{X}_{\sigma_{0}}(\mathbf{G}) \times \mathbf{X}_{\sigma_{0}}(\mathbf{G})$ and $f$ restricts to an isomorphism from the analytic germ of $\mathrm{M}$ along $i\left(\mathrm{X}_{\sigma_{0}}(\mathbf{G})\right)$ onto $\mathrm{G}^{\text {an }}\left(\mathrm{X}_{\sigma_{0}}, \mathrm{~F}_{\sigma_{0}}\right)$.

Roughly speaking, condition ii) means that the germ $\mathrm{G}^{a n}\left(\mathrm{X}_{\sigma_{0}}, \mathrm{~F}_{\sigma_{0}}\right)$ of analytic manifold along the diagonal in $\mathrm{X}_{\sigma_{0}}(\mathbf{G}) \times \mathrm{X}_{\sigma_{0}}(\mathbf{G})$ "extends" to a submanifold of $\mathrm{X}_{\sigma_{0}}(\mathbf{G}) \times \mathrm{X}_{\sigma_{0}}(\mathbf{G})$ which satisfies the Liouville property. It holds for instance when the holonomy groupoid of the holomorphic foliation on $\mathrm{X}_{\sigma_{0}}(\mathbf{G})$ defined by $\mathrm{F}_{\sigma_{0}}$ satisfies the Liouville property.

The necessity of conditions i) and ii) follows again easily from the properties of the $p$-curvature discussed above and from the fact that smooth connected complex algebraic varieties satisfy the Liouville property, by considering the normalization of the Zariski closure of $\mathrm{G}^{\text {for }}(\mathrm{X}, \mathrm{F})$. Their sufficiency will also be derived from Theorem 3.4 in Section 3.4.3.

\subsection{Algebraic Lie algebras and restricted Lie algebras}

\subsubsection{Algebraic subgroups and Lie subalgebras closed under p-th powers}

Let $\mathrm{G}$ be an algebraic group over a number field $\mathrm{K}$.

For any sufficiently divisible integer $\mathrm{N}$, there exists a model $\mathscr{G}$ of $\mathrm{G}$, i.e., a smooth quasi-projective group scheme over $\mathrm{S}:=\operatorname{Spec} \mathscr{O}_{\mathrm{K}}[1 / \mathrm{N}]$ whose generic fiber $\mathscr{G}_{\mathrm{K}}$ coincides with $\mathrm{G}$ (as $\mathrm{K}$-algebraic group). The restriction to the zero-section of $\mathscr{G}$ of the relative tangent bundle $\mathrm{T}_{\mathscr{G} / \mathrm{S}}$ defines the Lie algebra Lie $\mathscr{G}$ of $\mathscr{G}$ : it is a finitely generated projective module and a Lie algebra over $\mathscr{O}_{\mathrm{K}}[1 / \mathrm{N}]$, and the K-Lie algebra $(\text { Lie } \mathscr{G})_{\mathrm{K}}$ may be identified with Lie G. Moreover, for every non-zero prime ideal $\mathbf{p}$ of $\mathscr{C}_{\mathrm{K}}[1 / \mathrm{N}]$ with residue field $\mathbf{F}_{\mathbf{p}}$ of characteristic $p$, the $\mathbf{F}_{\mathbf{p}}$-Lie algebra $(\text { Lie } \mathscr{G})_{\mathbf{F}_{\mathbf{p}}}$ is canonically isomorphic to the Lie algebra of the smooth algebraic group $\mathscr{G}_{\mathrm{F}_{\mathrm{p}}}$ over the finite field $\mathbf{F}_{\mathbf{p}}$, and is therefore endowed with a $p$-th power map, given by the restriction of the $p$-th power map on global sections of $\mathrm{T}_{\mathscr{S}_{\mathbf{F}_{\mathbf{p}}}}$ to the left-invariant ones.

For instance, if $\mathrm{G}$ is the multiplicative group $\mathbf{G}_{m, \mathrm{~K}}$, we may choose $\mathscr{G}:=\mathbf{G}_{m}, \mathscr{O}_{\mathrm{K}}$ as model of $\mathrm{G}$ over $\operatorname{Spec} \mathscr{C}_{\mathrm{K}}$. Let $\mathrm{X}$ denote the tautological coordinate on $\mathbf{G}_{m}$, defined by the inclusion morphism $\mathbf{G}_{m} \hookrightarrow \mathbf{A}^{1}$. The $\mathscr{O}_{\mathrm{K}}$-Lie algebra Lie $\mathscr{G}$ is freely generated by the invariant derivation $\mathrm{D}:=\mathrm{X} \frac{\partial}{\partial \mathrm{X}}$. The image $\mathrm{D}_{\mathbf{p}}$ of $\mathrm{D}$ in Lie $\mathscr{G}_{\mathbf{F}_{\mathrm{p}}}$ defines a base of Lie $\mathscr{G}_{\mathbf{F}_{\mathrm{p}}}$, and the $p$-th power map on Lie $\mathscr{G}_{\mathbf{F}_{\mathbf{p}}}$ is the $p$-linear endomorphism which maps $\mathrm{D}_{\mathbf{F}_{\mathbf{p}}}$ to itself.

If $\mathrm{G}$ is an elliptic curve $\mathrm{E}$ over $\mathbf{Q}$ which admits good reduction over $\mathbf{Z}[1 / \mathrm{N}]$, and if $\mathscr{G}$ is the elliptic curve $\mathscr{E}$ over $\operatorname{Spec} \mathbf{Z}[1 / \mathrm{N}]$ which extends $\mathrm{E}$, then the $p$-th power map on Lie $\mathscr{G}_{\mathbf{F}_{p}}=$ Lie $\mathscr{E}_{\mathbf{F}_{p}}$ is the multiplication by the class in $\mathbf{F}_{p}$ of

$$
a_{p}(\mathrm{E}):=p+1-\left|\mathscr{E}\left(\mathbf{F}_{p}\right)\right| .
$$


This follows from the basic properties of the Hasse invariant of $\mathscr{G}_{\mathbf{F}_{p}}{ }^{(2)}$.

Theorem 2.3. - For any Lie subalgebra $\mathbf{h}$ of Lie $\mathrm{G}$ (defined over $\mathrm{K}$ ), the following two conditions are equivalent:

i) For almost every non-zero prime ideal $\mathbf{p}$ of $\mathscr{O}_{\mathrm{K}}[1 / \mathrm{N}]$, the $\mathbf{F}_{\mathbf{p}}$-Lie subalgebra $(\mathbf{h} \cap \text { Lie } \mathscr{G})_{\mathbf{F}_{\mathbf{p}}}$ of Lie $\mathscr{G}_{\mathbf{F}_{\mathbf{p}}}$ is closed under p-th powers.

ii) There exists an algebraic subgroup $\mathrm{H}$ of $\mathrm{G}$ (defined over $\mathrm{K}$ ) such that $\mathbf{h}=\mathrm{Lie} \mathrm{H}$.

Proof. - To prove the implication i) $\Rightarrow$ ii), we apply Theorem 2.1 to the situation where $\mathrm{X}$ is $\mathrm{G}, \mathrm{P}$ is the neutral element $e$ of $\mathrm{G}(\mathrm{K})$, and $\mathrm{F}$ is the left-invariant subbundle of $\mathrm{T}_{\mathrm{G}}$ whose fiber at $e$ is the subspace $\mathbf{h}$ of Lie $\mathrm{G}$. Since $\mathbf{h}$ is a Lie subalgebra of Lie $\mathrm{G}$, this subbundle is involutive. We may take $\mathscr{G}$ as model $\mathscr{C}$ of $\mathrm{X}=\mathrm{G}$, and define $\mathscr{F}$ as the saturated subsheaf of $\mathrm{T}_{\mathscr{G}} / \mathrm{s}$ such that $\mathscr{F}_{\mathrm{K}}=\mathrm{F}$. Condition i) in Theorem 2.1 then follows from (indeed, is equivalent to) condition i) in Theorem 2.3. Moreover, condition ii) in Theorem 2.1 is satisfied for any embedding $\sigma_{0}: \mathbf{K} \hookrightarrow \mathbf{G}$; indeed, condition ii) ${ }^{\prime}$ is satisfied by the restriction to the subspace $\mathbf{h}_{\sigma_{0}}$ of Lie $G_{\sigma_{0}}$ of the exponential map of the complex Lie group $G_{\sigma_{0}}(\mathbf{C})$. Therefore Theorem 2.1 shows that, if condition i) holds, then the leaf of $\mathrm{F}$ through $e$ is algebraic; this algebraic leaf provides the required algebraic subgroup $\mathrm{H}$ of $\mathrm{G}$.

The implication ii) $\Rightarrow$ i) follows from the existence of a smooth model $\mathscr{H}$ in $\mathscr{G}$ of the subgroup $\mathrm{H}$ of $\mathrm{G}$ (possibly after increasing $\mathrm{N}$ ). Then the Lie algebra $\mathbf{h} \cap$ Lie $\mathscr{G}$ is canonically isomorphic with Lie $\mathscr{H}$, and its reduction modulo $\mathbf{p}$ gets identified with the $\mathbf{F}_{\mathbf{p}}$-Lie subalgebra Lie $\mathscr{H}_{\mathbf{F}_{\mathrm{p}}}$ of Lie $\mathscr{G}_{\mathbf{F}_{\mathrm{p}}}$, defined by the smooth algebraic subgroup $\mathscr{H}_{\mathbf{F}_{\mathrm{p}}}$ of $\mathscr{G}_{\mathbf{F}_{\mathrm{p}}}$, and is therefore stable under $p$-th powers.

Observe that conditions i) and ii) in Theorem 2.3 are also equivalent to the following one:

iii) The formal Lie subgroup $\exp \mathbf{h}$, with Lie algebra $\mathbf{h}$, of the formal completion $\hat{\mathrm{G}}_{e}$ of $\mathbf{G}$ at the neutral element e extends to a formal Lie subgroup of the completion $\hat{\mathscr{G}}_{e}$ over $\operatorname{Spec} \mathscr{C}_{\mathrm{K}}\left[1 / \mathrm{N}^{\prime}\right]$ for some large enough integer $\mathrm{N}^{\prime}$.

Indeed, we clearly have ii) $\Rightarrow$ iii) $\Rightarrow$ i).

When $\mathrm{G}$ is an abelian variety, the implication iii) $\Rightarrow$ i) is basically due to Graftieaux ${ }^{(3)}$.

Let us emphasize the arithmetic significance of Theorem 2.3. For instance, applied to the algebraic group $\mathrm{G}:=\mathbf{G}_{m, \mathrm{~K}} \times \mathbf{G}_{m, \mathrm{~K}}$ over some number field $\mathrm{K}$ and

(2) For a "modern" proof, see for instance [KM85], (12.4.1.2) and (12.4.1.3), and [Kat73], (3.2.1).

(3) See [Gr0la] and [Gr0lb]; actually, Graftieaux's results hold under additional hypotheses - they involve semistable Néron models and require the extension of $\exp \mathbf{h}$ to exist over $\operatorname{Spec} \mathscr{O}_{\mathrm{K}}-$ but, when these are satisfied, provide quantitative statements. 
to the Lie subalgebra $\mathbf{h}$ of Lie $\mathrm{G}$ defined as the graph of the multiplication by some scalar $\lambda \in \mathrm{K}$ in Lie $\mathbf{G}_{m, \mathrm{~K}}$, it amounts to the following statement, which goes back to Kronecker ([Kro80]) and is indeed a consequence of Čebotarev's density theorem (see also [CC85a], Section 4):

Corollary 2.4. - If $\lambda$ is an element of a number field $\mathrm{K}$ such that, for almost every non-zero prime ideal $\mathbf{p}$ of $\mathscr{O}_{\mathrm{K}}$, its class in $\mathbf{F}_{\mathbf{p}}$ belongs to the prime field $\mathbf{F}_{p}$, then $\lambda$ belongs to $\mathbf{Q}$.

Proof. - The description of the $p$-th power map on Lie $\mathbf{G}_{m, \mathbf{F}_{\mathbf{p}}}$ recalled above shows that the reduction of $\mathbf{h}$ modulo $\mathbf{p}$ is stable under $p$-th power if and only if the class of $\lambda$ in $\mathbf{F}_{\mathbf{p}}$ belongs to the prime field $\mathbf{F}_{p}$. Besides, $\mathbf{h}$ is algebraic if and only if $\lambda$ belongs to $\mathbf{Q}$ since one-dimensional connected algebraic subgroups of $\mathbf{G}_{m, \mathrm{~K}} \times \mathbf{G}_{m, \mathrm{~K}}$ are defined by one equation of the form $\mathrm{X}^{a} \mathrm{Y}^{b}=1$, where $(a, b)$ is a pair of coprime integers.

When $\mathrm{K}$ is $\mathbf{Q}$ and $\mathrm{G}$ is the product $\mathrm{E} \times \mathrm{E}^{\prime}$ of two elliptic curves $\mathrm{E}$ and $\mathrm{E}^{\prime}$ over $\mathbf{Q}$, then Theorem 2.3 boils down to the following special case of Faltings isogeny theorem ([Fal83], §5, Corollary 2; see also [Ser68], IV.2.3, for the case where $j(\mathrm{E}) \notin \mathbf{Z})$ :

Corollary 2.5. - For any two elliptic curves $\mathrm{E}$ and $\mathrm{E}^{\prime}$ over $\mathbf{Q}$ the following conditions are equivalent:

1) $\mathrm{E}$ and $\mathrm{E}^{\prime}$ are $\mathbf{Q}$-isogenous.

2) For almost every prime number $p$,

$$
a_{p}(\mathbf{E})=a_{p}\left(\mathbf{E}^{\prime}\right)
$$

Proof. - Let $\mathbf{h}$ be any one-dimensional $\mathbf{Q}$-vector subspace of Lie $\mathrm{G}=\operatorname{Lie} \mathrm{E} \oplus$ Lie $\mathrm{E}^{\prime}$, distinct from Lie $\mathrm{E} \oplus\{0\}$ and $\{0\} \oplus$ Lie $\mathrm{E}^{\prime}$. Choose $\mathrm{N}$ sufficiently divisible, and define $\mathscr{G}$ as the product of the two elliptic curves $\mathscr{E}$ and $\mathscr{E}^{\prime}$ over $\mathbf{Z}[1 / \mathrm{N}]$ which respectively extend $\mathrm{E}$ and $\mathrm{E}^{\prime}$. Then, for any prime $p$ which does not divide $\mathrm{N}$, the fact that the $p$-th power map on Lie $\mathscr{E}_{\mathbf{F}_{p}}$ (resp. on Lie $\mathscr{E}^{\prime}{ }_{\mathbf{F}_{p}}$ ) is given by the multiplication by $a_{p}(\mathbf{E})$ (resp. by $a_{p}\left(\mathbf{E}^{\prime}\right)$ ) shows that the reduction of $\mathbf{h}$ modulo $p$ is stable under $p$-th power if and only if (2.1) holds modulo $p$. Moreover, if $\mathbf{h}$ is algebraic, the algebraic subgroup $\mathrm{H}$ of $\mathrm{E} \times \mathrm{E}^{\prime}$ such that $\mathbf{h}=\mathrm{Lie} \mathrm{H}$ is $\mathbf{Q}$-isogenous both to $\mathrm{E}$ and $\mathrm{E}^{\prime}$. This shows that the implication 2) $\Rightarrow 1$ ) is a straightforward consequence of Theorem 2.3. The converse implication 1$) \Rightarrow 2$ ) is well known ${ }^{(4)}$.

In their paper [CC85b] - which indeed has been one the main motivations of the present work - D. V. and G. V. Chudnovsky present a proof of a statement

(4) In fact, the previous construction (applied to the Lie algebra $\mathbf{h}$ of the graph of an isogeny from $\mathrm{E}$ to $\mathrm{E}^{\prime}$ ) shows that condition 1) implies that (2.1) holds modulo $p$ for almost every prime $p$; the equality (2.1) in $\mathbf{Z}$ then follows for $p$ large enough from the Hasse bound $\left|a_{p}(\mathrm{E})\right| \leqslant 2 \sqrt{p}$. 
similar to Corollary 2.5, based on an algebraicity criterion which is a special case of our Theorem 3.4. However, their proof requires more delicate results of Honda concerning the formal groups (over $\mathbf{Z}$ ) of Néron models of elliptic curves over $\mathbf{Q}(c f$. [Hon68]), which are not needed in our approach.

\subsubsection{Connected algebraic groups up to isogeny over number fields}

It is convenient to reformulate Theorem 2.3 in terms of the category of algebraic groups up to isogeny.

For any number field $\mathrm{K}$, let $\mathbf{G r}(\mathrm{K})$ be the category whose objects are the connected algebraic groups over $\mathrm{K}$ and whose morphisms are the morphisms of algebraic groups over $\mathrm{K}$. We may consider the class of morphisms in $\mathbf{G r}(\mathrm{K})$ formed by the isogenies ${ }^{(5)}$, and the localization $\mathbf{G r}_{\text {isog }}(\mathbf{K})$ of $\mathbf{G r}(\mathbf{K})$ by this class. It is easily checked that the class of isogenies admits a calculus of right fractions, and that $\mathbf{G r}_{\text {isog }}(\mathbf{K})$ may also be described as the category with the same objects as $\mathbf{G r}(\mathbf{K})$, in which the morphisms from a connected algebraic K-group $G_{1}$ to another one $G_{2}$ are the K-Lie algebra morphisms from Lie $G_{1}$ to Lie $G_{2}$, the graphs of which are algebraic Lie subalgebras of $G_{1} \times G_{2}$ (the composition of morphisms being the obvious one). When $\mathrm{G}_{1}$ and $\mathrm{G}_{2}$ are commutative, this set of morphisms $\operatorname{Hom}_{\mathbf{G r}_{\text {isog }}(\mathrm{K})}\left(\mathrm{G}_{1}, \mathrm{G}_{2}\right)$ is naturally an abelian group (use the addition law on $\mathrm{G}_{2}$ ) and may be identified with the group $\operatorname{Hom}_{\mathbf{G r}(\mathrm{K})}\left(\mathrm{G}_{1}, \mathrm{G}_{2}\right) \otimes_{\mathbf{Z}} \mathbf{Q}$ (thus we recover the classical definition of "morphisms up to isogeny" from $G_{1}$ to $G_{2}$ ). We shall denote by $\mathbf{G r C o m}_{\text {isog }}(K)$ the full subcategory of $\mathbf{G r}_{\text {isog }}(\mathrm{K})$, the objects of which are the commutative connected algebraic groups over K.

Let us introduce the category $\operatorname{LieRest}_{\mathbf{a}}(\mathbf{K})$ in which an object is a finite dimensional K-Lie algebra, equipped, for almost every prime ideal $\mathbf{p}$ of $\mathrm{K}$, of a $p$-th power map on its reduction modulo $\mathbf{p}$ which makes it a restricted Lie algebra, and morphisms are K-Lie algebra morphisms, almost all reductions of which are morphisms of restricted Lie algebras. Finally let $\mathbf{F r o b}_{\mathbf{a e}}(\mathbf{K})$ be the full subcategory of LieRest $_{\mathbf{a}}(\mathrm{K})$ defined by the objects of this category with a commutative underlying K-Lie algebra ${ }^{(6)}$.

One defines a functor

$$
\text { Lie }: \mathbf{G r}_{\text {isog }}(\mathrm{K}) \longrightarrow \text { LieRest }_{\mathbf{a e}}(\mathrm{K}) \text {, }
$$

by sending a connected commutative algebraic group $\mathrm{G}$ over $\mathrm{K}$ to its Lie algebra, endowed with its natural $p$-th power maps on almost its reductions, and a morphism

(5) A morphism $\varphi: G_{1} \rightarrow G_{2}$ in $\mathbf{G r}(\mathrm{K})$ is an isogeny if and only if it is étale, or equivalently, if and only if its differential at the origin $\operatorname{Lie} \varphi: \operatorname{Lie}_{1} \rightarrow \operatorname{Lie}_{2}$ is an isomorphism.

(6) In other words, $\operatorname{Frob}_{\mathbf{a e}}(\mathrm{K})$ is the category of finite dimensional K-vector spaces, equipped, for almost every prime ideal $\mathbf{p}$ of $\mathrm{K}$, of a "Frobenius map", i.e., of a $p$-linear endomorphism of its reduction modulo $\mathbf{p}$; morphisms in $\operatorname{Frob}_{\mathbf{a e}}(\mathrm{K})$ are the K-linear maps, almost all reductions of which commute with the Frobenius maps. The category $\operatorname{Frob}_{\mathbf{a e}}(\mathrm{K})$ is clearly a $\mathbf{Q}$-linear abelian category. 
of algebraic groups between two such groups to its differential at the origin. By restriction, this functor defines a functor

$$
\text { Lie }: \operatorname{GrCom}_{\text {isog }}(\mathrm{K}) \longrightarrow \text { Frob }_{\mathbf{a e}}(\mathrm{K}) \text {. }
$$

Theorem 2.3 shows that any subobject of an object in the essential image of these functors still belongs to this essential image. Moreover, applied to graphs of linear maps between Lie algebras of algebraic groups over $\mathrm{K}$, it leads to:

Corollary 2.6. - The functors (2.2) and (2.3) are fully faithful.

Finally, let us indicate that, restricted to abelian varieties and Jacobians, the functor (2.3) is naturally isomorphic to functors having simple definitions in terms of coherent cohomology.

Indeed, for any abelian variety A over $\mathrm{K}$, the Lie algebra Lie A is canonically isomorphic to the cohomology group $\mathrm{H}^{1}\left(\hat{\mathrm{A}}, \mathscr{O}_{\hat{\mathrm{A}}}\right)$ of the dual abelian variety $\hat{\mathrm{A}}$. Moreover, if $\hat{b}$ denotes a model of $\hat{A}$ over a dense open subscheme of Spec $\mathscr{O}_{\mathrm{K}}$, then, for almost every prime ideal $\mathbf{p}$ of $\mathscr{C}_{\mathrm{K}}$, the reduction modulo $\mathbf{p}$ of Lie $\mathrm{A}$, equipped with the $p$-th power map, may be identified with $\mathrm{H}^{1}\left(\hat{\mathscr{b}}_{\mathbf{F}_{\mathbf{p}}}, \mathscr{O}_{\hat{b}_{\mathbf{F}_{\mathbf{p}}}}\right)$ equipped with the $p$-linear map defined by the Frobenius morphism Frob $: a \mapsto a^{p}$ on $\mathscr{C}_{\hat{b}_{\mathbf{F}}}$ (see [Mum70], Section 13, Corollary 3, and Section 15, Theorem 3).

In particular, if $\mathrm{G}$ is a smooth, projective, geometrically irreducible curve $\mathrm{C}$ over $\mathrm{K}$, the image of its $\operatorname{Jacobian} \mathrm{Jac}(\mathrm{C})$ by the functor (2.3) may be identified with $\mathrm{H}^{1}\left(\mathrm{C}, \mathscr{O}_{\mathrm{C}}\right)$ equipped with the $p$-linear maps defined by the Frobenius morphisms Frob: $\mathscr{C}_{\mathrm{C}_{\mathbf{F}_{\mathrm{p}}}} \mapsto \mathscr{O}_{\mathrm{C}_{\mathbf{F}_{\mathrm{p}}}}$ of its reductions $\mathrm{C}_{\mathbf{F}_{\mathrm{p}}}$ (use the canonical isomorphisms $\mathrm{Jac}(\mathrm{C})^{\wedge} \simeq \mathrm{Jac}(\mathrm{C})$ and $\left.\mathrm{H}^{1}\left(\mathrm{Jac}(\mathrm{C}), \mathscr{O}_{\mathrm{Jac}(\mathrm{C})}\right) \simeq \mathrm{H}^{1}\left(\mathrm{C}, \mathscr{O}_{\mathrm{C}}\right)\right)$.

More generally, for any effective divisor $\mathrm{D}$ on $\mathrm{C}$, the image under the functor (2.3) of the generalized Jacobian $\mathrm{Jac}_{\mathrm{D}}(\mathrm{G})$, which parametrizes line bundles $\mathrm{L}$ of degree 0 over $\mathrm{C}$ equipped with a trivialization of $\mathrm{L}_{\mid \mathrm{D}}$, may be identified with $\mathrm{H}^{1}\left(\mathrm{C}, \mathscr{C}_{\mathrm{C}}(-\mathrm{D})\right)$ equipped with the $p$-linear maps defined by the composition of the Frobenius morphisms Frob: $\mathscr{O}_{\mathrm{C}}(-\mathrm{D})_{\mathbf{F}_{\mathrm{p}}} \rightarrow \mathscr{O}_{\mathrm{G}}(-p \mathrm{D})_{\mathbf{F}_{\mathrm{p}}}$ and of the inclusions $\mathcal{O}_{\mathrm{C}}(-p \mathrm{D})_{\mathbf{F}_{\mathrm{p}}} \hookrightarrow \mathcal{O}_{\mathrm{C}}(-\mathrm{D})_{\mathbf{F}_{\mathrm{p}}}$.

\subsection{Principal G-bundles with flat connections}

In this section, we freely use the basic definitions concerning principal G-bundles and connections recalled in Appendix A.

\subsubsection{The conjecture of Grothendieck-Katz}

Recall that the algebraicity of leaves of the horizontal foliation attached to a flat connection on a smooth principal bundle may be interpreted as a finiteness property 
of its monodromy. Indeed, if $\mathrm{K}$ is a subfield of $\mathbf{C}, \mathrm{G}$ an algebraic group over $\mathrm{K}$, and $\mathrm{X}$ a principal $\mathrm{G}$-bundle over a smooth connected algebraic variety $\mathrm{B}$ over $\mathrm{K}$, equipped with a flat connection $\mathrm{H} \subset \mathrm{T}_{\mathrm{X} / \mathrm{B}}$, the following conditions are easily seen to be equivalent:

1. There exists a finite étale morphism $v: \mathrm{B}^{\prime} \rightarrow \mathrm{B}$ such that the pull-back by $v$ of the G-bundle with connection $(\mathrm{X}, \mathrm{H})$ over $\mathrm{B}$ is isomorphic to the trivial G-bundle with connection over $\mathrm{B}^{\prime}$.

2. After possibly replacing $\mathrm{K}$ by some finite extension, there exists a finite algebraic subgroup $\mathrm{H}$ of $\mathrm{G}$ over $\mathrm{K}$ and a principal $\mathrm{H}$-bundle $\mathrm{X}^{\prime}$ over $\mathrm{B}$ (defined over $\mathrm{K}$ ) such that, if $\varphi: \mathrm{H} \rightarrow \mathrm{G}$ denotes the inclusion morphism, the G-bundle ${ }^{\varphi} \mathrm{X}^{\prime}$, equipped with the flat connection deduced from the (unique, necessarily flat) connection on $\mathrm{X}^{\prime}$, is isomorphic to $(\mathrm{X}, \mathrm{H})$.

If moreover $x_{0}$ is a point in $\mathrm{X}(\mathrm{K})$, these conditions are equivalent to the following one:

3. The leaf of $H$ through $x_{0}$ is algebraic.

Finally, when the base variety $B$ is proper over $K$, or when the algebraic group $G$ is linear and the connection $\mathrm{H}$ has regular singular points, they are also equivalent to:

4. For some (or equivalently, for any) base point $x_{0} \in \mathbf{X}(\mathbf{G})$, with image $b_{0}:=p\left(x_{0}\right) \in \mathbf{B}(\mathbf{G})$, the associated monodromy representation $\pi_{1}\left(\mathbf{B}(\mathbf{C}), b_{0}\right) \rightarrow$ $\mathrm{G}(\mathbf{C})$, defined by the complex analytic integrable connection $\mathrm{H}_{\mathbf{C}}$ on the analytic principal $\mathrm{G}(\mathbf{G})$-bundle $\mathrm{X}(\mathbf{G})$ over $\mathrm{B}(\mathbf{G})$, has a finite image.

When the above conditions hold, we shall say that the G-bundle with connection $(\mathrm{X}, \mathrm{H})$ becomes trivial on some finite étale covering, or that the monodromy of $(\mathrm{X}, \mathrm{H})$ is algebraically finite. This is clearly a geometric property ${ }^{(7)}$. Moreover, when investigating criterions for finite algebraic monodromy, we may restrict to the situation where the base B is quasi-projective, or even is a curve. Indeed, standard techniques allow to prove the following proposition, which we leave as an exercise:

Proposition 2.7. - Let us keep the notation above, and let $\mathrm{U}$ be a Zariski open dense subscheme of $\mathrm{B}$ and $\mathrm{X}_{\mathrm{U}}$ its inverse image in $\mathrm{X}$. The following conditions are equivalent:

1) The monodromy of the flat $\mathrm{G}$-bundle with connection $(\mathrm{X}, \mathrm{H})$ is algebraically finite.

2) The monodromy of the flat G-bundle with connection $\left(\mathrm{X}_{\mathrm{U}}, \mathrm{H}_{\mathrm{X}_{\mathrm{U}}}\right)$ over $\mathrm{U}$ is algebraically finite.

3) For any smooth connected curve $\mathrm{G}$ over $\mathrm{K}$, which is a subscheme of $\mathrm{B}$, the monodromy of the pull-back to $\mathrm{C}$ of $(\mathrm{X}, \mathrm{H})$ is algebraically finite.

(7) Namely, if $\mathrm{K}^{\prime}$ is an extension of $\mathrm{K}$, equipped with an arbitrary embedding in $\mathbf{G}$, the G-bundle with connection $(\mathrm{X}, \mathrm{H})$, defined over $\mathrm{K}$, has finite algebraic monodromy if and only if the $\mathrm{G}_{\mathrm{K}^{\prime}}$-bundle with connection $\left(\mathrm{X}_{\mathrm{K}^{\prime}}, \mathrm{H}_{\mathrm{K}^{\prime}}\right)$, deduced from $(\mathrm{X}, \mathrm{H})$ by the base change Spec $\mathrm{K} \rightarrow$ Spec $\mathrm{K}^{\prime}$, also has finite algebraic monodromy. 
The conjecture of Grothendieck-Katz asserts that, when $\mathrm{K}$ is a number field and $\mathrm{G}$ is the linear group $\mathrm{GL}_{\mathrm{N}, \mathrm{K}}$ (or equivalently any linear algebraic group over $\mathrm{K}$ ), a flat $\mathrm{G}$-bundle with connection $(\mathrm{X}, \mathrm{H})$ becomes trivial on some finite étale covering iff, for almost every non-zero prime ideal $\mathbf{p}$ of $\mathscr{C}_{\mathrm{K}}$, of residue characteristic $p$, the p-curvature of the reduction modulo $\mathbf{p}$ of $(\mathrm{X}, \mathrm{H})$ vanishes. (See [Kat72]; see also [Kat82] and [And99]. Actually, the conjecture may be formulated over any field $\mathrm{K}$ of characteristic 0 , but reduces to the case of number fields by standard specialization techniques.) We will call generalized conjecture of Grothendieck-Katz the same statement where $\mathrm{G}$ is an arbitrary algebraic group.

Thanks to the work of D. V. and G. V. Chudnovsky and André, the conjecture of Grothendieck-Katz is known to hold when the differential Galois group of the flat linear bundle $(\mathrm{X}, \mathrm{H})$ has a solvable neutral component, or equivalently, for flat $\mathrm{G}$ bundles, when $G$ is a linear algebraic group over $K$ whose neutral component $G^{0}$ is solvable (see [CC85a], Section 8, and [And89], Chapter VIII, Exercises 5) and 6), when $\mathrm{G}=\mathbf{G}_{m}$ and [And99], Section 3, when $\mathrm{G}=\mathbf{G}_{a}$ and in general). In the next section, we present a derivation of this result - indeed of the generalized conjecture of Grothendieck-Katz for any algebraic group $G$ such that $G^{0}$ is solvable - as an application of our algebraicity criterion Theorem $2.1^{(8)}$.

Let us finally observe that the interpretation of the algebraic finiteness of the monodromy of a flat G-bundle in terms of the algebraicity of the leaves of its horizontal foliation shows that the generalized conjecture of Grothendieck-Katz is a special case of the conjecture $\mathrm{F}$ of Ekedahl and Shepherd-Barron ( $c f$. Introduction and [ESB99], Theorem 2.4). Together with Theorem 2.1, this interpretation also leads to the following special case of the generalized conjecture of Grothendieck-Katz:

Corollary 2.8. - With the above notation, a flat $\mathrm{G}$-bundle with connection $(\mathrm{X}, \mathrm{H})$ over a smooth geometrically connected $\mathrm{K}$-scheme $\mathrm{B}$ becomes trivial on some finite étale covering when the following two conditions are satisfied:

i) For almost every non-zero prime ideal $\mathbf{p}$ of $\mathscr{C}_{\mathrm{K}}$, of residue characteristic $p$, the p-curvature of the reduction modulo $\mathbf{p}$ of $(\mathrm{X}, \mathrm{H})$ vanishes.

ii) There exists an imbedding $\sigma_{0}: \mathbf{K} \hookrightarrow \mathbf{C}$ such that the complex manifold $\mathbf{B}_{\sigma_{0}}(\mathbf{G})$ has a universal covering which satisfies the Liouville property.

Observe that this last condition holds for instance when B is an algebraic group.

\subsubsection{Flat principal bundles with solvable structural groups}

The end of this section is devoted to the proof of the following statement:

(8) Besides technical differences in the proofs and the more general character of our theorem, it seems worthwhile to include a self-contained derivation of the results of Chudnovsky-André, which are not so well documented in spite of their importance. 
Theorem 2.9. - Let $\mathrm{K}$ be a number field, and $\mathrm{G}$ an algebraic group over $\mathrm{K}$ whose neutral component $\mathrm{G}^{0}$ is solvable. For any principal $\mathrm{G}$-bundle $\mathrm{X}$ over a smooth connected variety $\mathrm{B}$ over $\mathrm{K}$, equipped with a flat connection $\mathrm{H}$, the following conditions are equivalent:

i) For almost every non-zero prime ideal $\mathbf{p}$ of $\mathscr{C}_{\mathrm{K}}$, of residue characteristic $p$, the p-curvature of the reduction modulo $\mathbf{p}$ of $(\mathrm{X}, \mathrm{H})$ vanishes.

ii) The $\mathrm{G}-$ bundle with connection $(\mathrm{X}, \mathrm{H})$ becomes trivial on some finite étale covering.

The special case of Theorem 2.9 where $G$ is a linear algebraic group may be rephrased as the equivalence of conditions i) and ii) when $G$ is the group $G_{N}$ and when, for some embedding $\sigma: \mathrm{K} \hookrightarrow \mathbf{C}$, the image of the monodromy of $\left(\mathrm{X}_{\sigma}, \mathrm{H}_{\sigma}\right)$ contains a solvable subgroup of finite index (observe that, according to [Kat70], condition ii) implies that $(\mathrm{X}, \mathrm{H})$ has regular singular points, and that the Zariski closure of a solvable group is solvable; compare [Kat82], Proposition 5.2).

Observe that, as a solvable covering of a smooth complex algebraic curve may fail to satisfy the Liouville property (see for instance [Anc90] or [Gri99]), Theorem 2.9 is not an immediate consequence of Theorem 2.1. Besides the above Corollary 2.8 of Theorem 2.1 and the constructions of principal bundles with connections recalled in Appendix A.2, its proof will be based on the following analytic result, of independent interest.

Proposition 2.10. - Let $\mathrm{C}$ be a smooth connected complex algebraic curve, and, for any positive integer $k$, let $\mathrm{S}^{k} \mathrm{C}$ be its $k$-th symmetric power. Let us denote by $g$ the genus of the smooth projective completion $\overline{\mathrm{C}}$ of $\mathrm{C}$, by $\delta$ the cardinality of $\overline{\mathrm{C}} \backslash \mathrm{C}$, and let ${ }^{(9)} g^{\prime}:=g+\max (0, \delta-1)$. Then, for any $k \geqslant \max \left(2, g^{\prime}\right)$, "the" universal covering $\widetilde{\mathrm{S}^{k} \mathrm{C}}$ of the manifold of complex points of $\mathrm{S}^{k} \mathrm{C}$ satisfies the Liouville property.

Proof of Proposition 2.10. - Let D be an effective divisor on $\overline{\mathrm{C}}$, the support of which is $\overline{\mathrm{C}} \backslash \mathrm{C}$, and let $\mathrm{Jac}_{\mathrm{D}}(\overline{\mathrm{C}})$ be the generalized Jacobian of $\overline{\mathrm{C}}$ associated to the "modulus" D (see for instance [Ser59], Chapter V). It is the commutative connected algebraic group over $\mathbf{C}$ which classifies line bundles $\mathrm{L}$ of degree 0 over $\overline{\mathrm{C}}$ equipped with an isomorphism $\varphi: \mathrm{L}_{\mid \mathrm{D}} \rightarrow \mathscr{O}_{\mid \mathrm{D}}$. We have a canonical isomorphism

$$
\text { Lie } \operatorname{Jac}_{\mathrm{D}}(\overline{\mathrm{C}}) \simeq \mathrm{H}^{1}(\overline{\mathrm{C}}, \mathcal{O}(-\mathrm{D})) \text {. }
$$

In particular

$$
\begin{aligned}
d=\operatorname{dim} \operatorname{Jac}_{\mathrm{D}}(\overline{\mathbf{C}}) & =\operatorname{rkH}^{1}(\overline{\mathrm{C}}, \mathcal{O}(-\mathrm{D})) \\
& =g+\max (0, \operatorname{deg} \mathrm{D}-1) .
\end{aligned}
$$

\footnotetext{
in $\overline{\mathrm{C}} \backslash \mathrm{C}$.

(9) This number $g^{\prime}$ is nothing else than the arithmetic genus of the curve obtained from $\overline{\mathrm{C}}$ by identifying the points
} 
Moreover, for any point $\mathrm{P}_{0} \in \mathrm{C}(\mathbf{G})$, we may consider the "jacobian embedding"

$$
j_{\mathrm{P}_{0}}: \mathrm{G} \longrightarrow \mathrm{Jac}_{\mathrm{D}}(\overline{\mathrm{G}}),
$$

which maps any point $\mathrm{P} \in \mathrm{G}(\mathbf{G})$ to the class of the line bundle $\mathscr{O} \overline{\mathrm{C}}\left(\mathrm{P}-\mathrm{P}_{0}\right)$ equipped with the tautological isomorphism $\mathscr{O} \overline{\mathrm{C}}\left(\mathrm{P}-\mathrm{P}_{0}\right)_{\mid \mathrm{D}} \simeq \mathcal{O}_{\mid \mathrm{D}}$. The embedding $j_{\mathrm{P}_{0}}$ induces an isomorphism of homology groups:

$$
j_{\mathrm{P}_{0}, *}: \mathrm{H}_{1}(\mathbf{G}(\mathbf{G}), \mathbf{Z}) \longrightarrow \mathrm{H}_{1}\left(\mathrm{Jac}_{\mathrm{D}}(\overline{\mathbf{C}})(\mathbf{G}), \mathbf{Z}\right)
$$

which does not depend on $\mathrm{P}_{0}$ ( $c f$. [Ser59], V.19, Proposition 11).

The morphism

$$
\begin{gathered}
\mathrm{C}^{d} \quad \longrightarrow \quad \operatorname{Jac}_{\mathrm{D}}(\overline{\mathrm{C}}) \\
\left(x_{1}, \ldots, x_{d}\right) \longmapsto \sum_{i=1}^{d} j_{\mathrm{P}_{0}}\left(x_{i}\right)
\end{gathered}
$$

is invariant under the permutation action of the symmetric group $\mathfrak{S}_{d}$ on $\mathrm{C}^{d}$, and therefore factorizes through a morphism

$$
j_{\mathrm{P}_{0}}^{d}: \mathrm{S}^{d} \mathrm{C} \longrightarrow \operatorname{Jac}_{\mathrm{D}}(\overline{\mathrm{C}}) \text {, }
$$

which is birational ( $c f$. [Ser59], V.9, Théorème 1).

Besides, the morphism

$$
\pi_{1}\left(\mathrm{G}(\mathbf{G}), \mathrm{P}_{0}\right)^{d} \simeq \pi_{1}\left(\mathrm{C}^{d}(\mathbf{G}),\left(\mathrm{P}_{0}, \ldots, \mathrm{P}_{0}\right)\right) \longrightarrow \pi_{1}\left(\mathrm{~S}^{d} \mathrm{C}(\mathbf{C}),\left[\left(\mathrm{P}_{0}, \ldots, \mathrm{P}_{0}\right)\right]\right)
$$

induced by the quotient map $\mathrm{C}^{d}(\mathbf{G}) \rightarrow \mathrm{S}^{d}(\mathbf{G})$ is surjective, since the latter is totally ramified at $\left(\mathrm{P}_{0}, \ldots, \mathrm{P}_{0}\right)$. Therefore, if

$$
i_{\mathrm{P}_{0}}: \mathrm{C} \longrightarrow \mathrm{S}^{d} \mathrm{C}
$$

denotes the morphism defined by

$$
i_{\mathrm{P}_{0}}(x):=\left[\left(x, \mathrm{P}_{0}, \ldots, \mathrm{P}_{0}\right)\right] \text {, }
$$

the induced morphism between fundamental groups

$$
i_{\mathrm{P}_{0}, *}: \pi_{1}\left(\mathrm{C}(\mathbf{C}), \mathrm{P}_{0}\right) \longrightarrow \pi_{1}\left(\mathrm{~S}^{d} \mathrm{C}(\mathbf{G}),\left[\left(\mathrm{P}_{0}, \ldots, \mathrm{P}_{0}\right)\right]\right)
$$

is surjective.

Let us now assume that $d \geqslant 2$. Then, according to the preceding discussion, the fundamental group $\pi_{1}\left(\mathrm{~S}^{d} \mathrm{C}(\mathbf{C}),\left[\left(\mathrm{P}_{0}, \ldots, \mathrm{P}_{0}\right)\right]\right)$ is commutative, and, since $j_{\mathrm{P}_{0}}^{d} \circ i_{\mathrm{P}_{0}}=j_{\mathrm{P}_{0}}$, we 
get a commutative diagram,

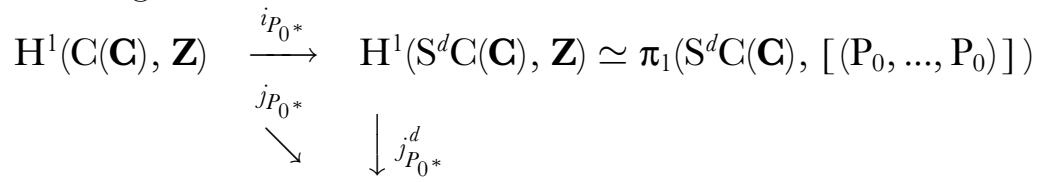

$$
\begin{aligned}
& \mathrm{H}_{1}\left(\mathrm{Jac}_{\mathrm{D}}(\overline{\mathbf{C}})(\mathbf{G}), \mathbf{Z}\right) \simeq \pi_{1}\left(\mathrm{Jac}_{\mathrm{D}}(\overline{\mathbf{C}})(\mathbf{G}), 0\right),
\end{aligned}
$$

in which all arrows are isomorphisms.

Moreover, the exponential

$$
\exp _{\mathrm{Jac}_{\mathrm{D}}(\overline{\mathbf{C}})(\mathbf{C})}: \operatorname{Lie} \mathrm{Jac}_{\mathrm{D}}(\overline{\mathbf{C}}) \longrightarrow \mathrm{Jac}_{\mathrm{D}}(\overline{\mathbf{C}})(\mathbf{G})
$$

of the connected commutative complex Lie group $\mathrm{Jac}_{\mathrm{D}}(\overline{\mathbf{G}})(\mathbf{G})$ is a universal covering. Since

$$
j_{\mathrm{P}_{0^{*}}}^{d}: \pi_{1}\left(\mathrm{~S}^{d} \mathrm{C}(\mathbf{G}),\left[\left(\mathrm{P}_{0}, \ldots, \mathrm{P}_{0}\right)\right]\right) \longrightarrow \pi_{1}\left(\mathrm{Jac}_{\mathrm{D}}(\overline{\mathbf{C}})(\mathbf{C}), 0\right)
$$

is an isomorphism, if we form the following cartesian diagram of analytic spaces:

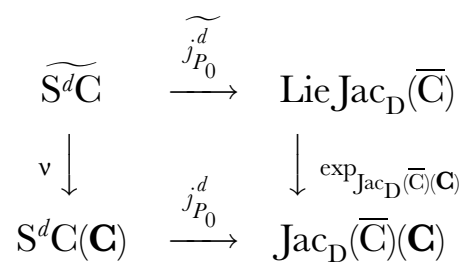

- in other words, $\widetilde{\mathrm{S}^{d} \mathrm{C}}$ is the complex manifold defined as the fibered product of $\mathrm{S}^{d} \mathrm{C}(\mathbf{G})$ and Lie $\operatorname{Jac}_{\mathrm{D}}(\overline{\mathbf{G}})$ over $\operatorname{Jac}_{\mathrm{D}}(\overline{\mathbf{G}})(\mathbf{G})$ - then

$$
v: \widetilde{\mathrm{S}^{d} \mathrm{C}} \longrightarrow \mathrm{S}^{d} \mathrm{C}(\mathbf{C})
$$

is also a universal covering. Let $\mathrm{U}$ (resp. V) be a Zariski open dense subvariety of $\mathrm{S}^{d} \mathrm{C}$ (resp. of $\mathrm{Jac}_{\mathrm{D}}(\overline{\mathrm{C}})$ ) such that $j_{\mathrm{P}_{0}}^{d}$ induces an isomorphism from $\mathrm{U}$ to $\mathrm{V}$. Then $\widetilde{j_{\mathrm{P}_{0}}^{d}}$ maps $\mathbf{v}^{-1}(\mathbf{U}(\mathbf{G}))$ biholomorphically onto $\exp _{\mathrm{Jac}_{\mathrm{D}}(\overline{\mathrm{C}})(\mathbf{C})}^{-1}(\mathrm{~V}(\mathbf{G}))$, and $\widetilde{\mathrm{S}^{d} \mathrm{C}} \backslash \mathrm{v}^{-1}(\mathrm{U}(\mathbf{G}))$ (resp. Lie Jac $\left.(\overline{\mathrm{D}}) \backslash \exp _{\mathrm{Jac}_{\mathrm{D}}(\overline{\mathrm{C}})(\mathbf{C})}^{-1}(\mathrm{~V}(\mathbf{C}))\right)$ is a closed strict analytic - hence pluripolar and Lebesgue null - subset of $\widetilde{\mathrm{S}^{d} \mathrm{C}}\left(\right.$ resp. of $\left.\mathrm{Lie} \mathrm{Jac}_{\mathrm{D}}(\overline{\mathrm{G}})\right)$. Since the complex vector space $\operatorname{Lie~Jac}_{\mathrm{D}}(\overline{\mathrm{C}})$ satisfies the Liouville property, this shows that the complex manifolds $\exp _{\mathrm{Jac}_{\mathrm{D}}(\overline{\mathrm{C}})(\mathbf{C})}^{-1}(\mathrm{~V}(\mathbf{G})), v^{-1}(\mathbf{U}(\mathbf{C}))$, and therefore $\widetilde{\mathrm{S}^{d} \mathrm{C}}$ satisfy it also.

When $\mathrm{C} \neq \overline{\mathrm{C}}$, any integer $k \geqslant \max \left(2, g^{\prime}\right)$ may be realized as the integer $d$ for a suitable choice of the divisor $\mathrm{D}$; therefore, Proposition 2.10 is established in that case.

When $\mathrm{G}=\overline{\mathrm{C}}$, we may apply the previous case to the curve $\mathrm{C}^{\prime}:=\mathrm{G} \backslash\left\{\mathrm{Q}_{0}\right\}$, where $Q_{0}$ is any point in $\mathrm{C}(\mathbf{C})$. Observe that $\mathrm{C}^{\prime}$ has the same invariants $g$ and $g^{\prime}$ as $\mathrm{C}$. 
Therefore, if $k \geqslant \max \left(2, g^{\prime}\right)$, the universal covering $\widetilde{\mathrm{S}^{d} \mathrm{C}^{\prime}}$ satisfies the Liouville property, and so does the image of a lifting $\widetilde{\mathrm{S}^{d} \mathrm{C}^{\prime}} \rightarrow \widetilde{\mathrm{S}^{d} \mathrm{C}}$ of the inclusion map $\mathrm{S}^{d} \mathrm{C}^{\prime} \hookrightarrow \mathrm{S}^{d} \mathrm{C}$ to universal coverings. Since this image is the inverse image in $\widetilde{\mathrm{S}^{d} \mathrm{C}}$ of the Zariski open subvariety $\mathrm{S}^{d} \mathrm{C}^{\prime} \subset \mathrm{S}^{d} \mathrm{C}$, hence open in $\mathrm{S}^{d} \mathrm{C}$ with Lebesgue null complement, this shows that $\widetilde{\mathrm{S}^{d} \mathrm{C}}$ also satisfies the Liouville property.

Proof of Theorem 2.9. - The implication ii) $\Rightarrow$ i) holds for any structural group G: this is a straightforward consequence of the functoriality properties of the $p$-curvatures with respect to inverse images.

To prove the direct implication i) $\Rightarrow$ ii), observe that, if $\varphi: G \rightarrow G^{\prime}$ is a morphism of algebraic K-groups with kernel $\mathrm{G}^{\prime \prime}$, and if the generalized conjecture of Grothendieck-Katz holds for $\mathrm{G}^{\prime}$-bundles, then, to prove it for G-bundles, we only need to prove it for $\mathrm{G}^{\prime \prime}$-bundles. This shows that, to prove Theorem 2.9, we may assume that $G$ is connected (consider the morphism $G \rightarrow G / G^{0}$ ), and then that $G$ is commutative (use induction on the length of a composition series with commutative subquotients of $\left.\mathrm{G}^{0}\right)$.

Moreover, according to Proposition 2.7, we may assume that the base B is a curve. After possibly replacing $\mathrm{K}$ by a finite extension $\mathrm{K}^{\prime}$ and $\mathrm{B}_{\mathrm{K}^{\prime}}$ by a component, we may also suppose that there exists some rational point $x_{0} \in \mathrm{X}(\mathrm{K})$.

Finally, to prove the implication i) $\Rightarrow$ ii) in Theorem 2.9, we may - and will assume that the structural group $\mathrm{G}$ is connected and commutative, that the base of the bundle $\mathrm{X}$ is a smooth geometrically connected curve $\mathrm{G}$ over $\mathrm{K}$, and that there exists rational points $x_{0} \in \mathrm{X}(\mathrm{K})$ and $b_{0}:=p\left(x_{0}\right) \in \mathrm{G}(\mathbf{K})$.

Consider now, for any positive integer $k$, the principal $\mathrm{G}^{k}$-bundle

$$
\begin{aligned}
p^{k}: \quad \mathrm{X}^{k} & \longrightarrow \mathrm{C}^{k} \\
\left(x_{1}, \ldots, x_{k}\right) & \longmapsto\left(p\left(x_{1}\right), \ldots, p\left(x_{k}\right)\right),
\end{aligned}
$$

and let us use the constructions of principal bundles and connections recalled in Appendix A.2. Since the "sum morphism"

$$
\begin{aligned}
& \Sigma^{k}: \quad \mathrm{G}^{k} \longrightarrow \mathrm{G} \\
& \left(g_{1}, \ldots, g_{k}\right) \longmapsto g_{1}+\ldots+g_{k}
\end{aligned}
$$

is a morphism of $\mathrm{K}$-algebraic groups, we may form the principal G-bundle ${ }^{\Sigma^{k}}\left(\mathrm{X}^{k}\right)$ over $\mathrm{C}^{k}$. The permutation action of the symmetric group $\mathfrak{S}_{k}$ on $\mathrm{X}^{k}$ descends to a left-action of $\mathfrak{S}_{k}$ on $\Sigma^{k}\left(\mathrm{X}^{k}\right)$, which commutes with the right-action of $\mathrm{G}$. Moreover, if $\mathrm{C}^{k}$ also is equipped with the permutation action of $\mathfrak{S}_{k}$, the structural morphism

$$
\Sigma^{k}\left(p^{k}\right): \Sigma^{k}\left(\mathrm{X}^{k}\right) \longrightarrow \mathrm{C}^{k}
$$

is $\mathfrak{S}_{k}$-equivariant and - locally for the étale topology - admits $\mathfrak{S}_{k}$-invariant sections (constructed from local - in the étale topology - sections of $p: \mathrm{X} \rightarrow \mathrm{C}$ ). Therefore, 
by quotienting through $\mathfrak{S}_{k}$, we get a principal G-bundle

$$
p_{k}: \mathrm{X}_{k}:=\mathfrak{S}_{k} \backslash \Sigma^{k}\left(\mathrm{X}^{k}\right) \longrightarrow \mathrm{S}^{k} \mathrm{C}:=\mathfrak{S}_{k} \backslash \mathrm{C}^{k} .
$$

The subbundle $\mathrm{H}^{\boxplus k}$ of the tangent bundle $\mathrm{T}_{\mathrm{X}^{k}} \simeq \mathrm{T}_{\mathrm{X}}^{\boxplus k}$ is a connection on the principal $\mathrm{G}^{k}$-bundle $p^{k}: \mathrm{X}^{k} \rightarrow \mathrm{C}^{k}$. The associated connection $\Sigma^{k} \mathrm{H}^{\boxplus k}$ on $\Sigma^{k}\left(\mathrm{X}^{k}\right)$ is $\mathfrak{S}_{k^{-}}$ invariant, and therefore descends to a connection $\mathrm{H}_{k}$ on $\mathrm{X}_{k}$. Moreover, the connection $\mathrm{H}^{\boxplus k}$, and consequently the connections $\Sigma^{\Sigma^{k}} \mathrm{H}^{\boxplus k}$ and $\mathrm{H}_{k}$, are flat, and almost all their reductions modulo a prime ideal $\mathbf{p}$ of $\mathscr{O}_{\mathrm{K}}$ have vanishing $p$-curvatures.

According to Corollary 2.8 and Proposition 2.10, if $k$ is large enough, then the G-bundle with connection $\left(\mathrm{X}_{k}, \mathrm{H}_{k}\right)$ becomes trivial on some finite étale covering. Since the G-bundle with connection $(\mathrm{X}, \mathrm{H})$ over $\mathrm{G}$ is isomorphic to the inverse image of $\left(\mathrm{X}_{k}, \mathrm{H}_{k}\right)$ by the embedding

$$
\begin{aligned}
i_{b_{0}}: \mathrm{C} & \longrightarrow \mathrm{S}^{k} \mathrm{C} \\
t & \longmapsto\left[\left(t, b_{0}, \ldots, b_{0}\right)\right],
\end{aligned}
$$

this proves that $(\mathrm{X}, \mathrm{H})$ also becomes trivial on some finite étale covering.

\section{Algebraization of formal germs of subvarieties in algebraic varieties over number fields}

\subsection{Size of smooth formal subschemes}

\subsubsection{Local theory}

Notation and definitions. - Let $k$ be a field equipped with a complete nonarchimedean absolute value ||$: k \rightarrow \mathbf{R}_{+}$and let $\mathscr{O}:=\{t \in k|| t \mid \leqslant 1\}$ be its valuation ring.

If $g:=\sum_{\mathbf{I} \in \mathbf{N}^{d}} a_{\mathrm{I}} \mathrm{X}^{\mathrm{I}}$ is a formal power series in $k\left[\left[\mathrm{X}_{1}, \ldots, \mathrm{X}_{d}\right]\right]$ and if $r \in \mathbf{R}_{+}^{*}$, we define

$$
\|g\|_{r}:=\sup _{\mathrm{I}}\left|a_{\mathrm{I}}\right| r^{|\mathrm{I}|} \in \mathbf{R}_{+} \cup\{+\infty\}
$$

The "norm" $\|g\|_{r}$ is finite if and only if the series $g$ is convergent and bounded on the open $d$-dimensional ball of radius $r$ in $\bar{k}^{d}$.

The group Aut $\hat{\mathbf{A}}_{k}^{d}$ of automorphisms of $\hat{\mathbf{A}}_{k}^{d}$, the formal completion at the origin of the $d$-dimensional affine space over $k$, may be identified with the space of $d$-tuples $f=\left(f_{i}\right)_{1 \leqslant i \leqslant d}$ of formal series $f_{i} \in k\left[\left[x_{1}, \ldots, x_{d}\right]\right]$ such that $f(0)=0$ and $\mathrm{D} f(0):=\left(\frac{\partial f_{i}}{\partial x_{j}}(0)\right)_{1 \leqslant i, j \leqslant d}$ belongs to $\mathrm{GL}_{n}(k)$. We may introduce its following subgroups: 
- the subgroup $\mathrm{G}_{\text {for }}$ formed by the formal automorphisms $f$ such that $\mathrm{D} f(0)$ belongs to $\mathrm{GL}_{n}(\odot)$;

- the subgroup $\mathrm{G}_{\omega}$ formed by the elements $f:=\left(f_{i}\right)_{1 \leqslant i \leqslant d}$ of $\mathrm{G}_{f o r}$ such that the series $f_{i}$ have positive radii of convergence;

- for any $r \in \mathbf{R}_{+}^{*}$, the subgroup $\mathrm{G}_{\omega}(r)$ of $\mathrm{G}_{\omega}$ formed by the elements $f:=\left(f_{i}\right)_{1 \leqslant i \leqslant d}$ of $\mathrm{G}_{\text {for }}$ such that the series $\left(f_{i}\right)_{1 \leqslant i \leqslant d}$ satisfy the bounds $\left\|f_{i}\right\|_{r} \leqslant r$. This group may be seen as the group of analytic automorphisms, preserving the origin, of the open $d$-dimensional ball of radius $r$. Moreover,

$$
r^{\prime}>r>0 \Rightarrow \mathrm{G}_{\omega}\left(r^{\prime}\right) \subset \mathrm{G}_{\omega}(r) \text { and } \bigcup_{r>0} \mathrm{G}_{\omega}(r)=\mathrm{G}_{\omega} .
$$

Size of a smooth formal subscheme in an affine space. — Let $\hat{\mathrm{V}}$ be a formal subscheme of $\hat{\mathbf{A}}_{k}^{d}$. For any $\varphi$ in Aut $\hat{\mathbf{A}}_{k}^{d}$, we may consider its inverse image $\varphi^{*}(\hat{\mathrm{V}})$, which is again a formal subscheme of $\hat{\mathbf{A}}_{k}^{d}$. Moreover, the following conditions are equivalent:

1. $\hat{\mathrm{V}}$ is a smooth formal scheme of dimension $v$.

2. There exists $\boldsymbol{\varphi}$ in Aut $\hat{\mathbf{A}}_{k}^{d}$ such that $\boldsymbol{\varphi}^{*}(\hat{\mathbf{V}})$ is the formal subscheme $\hat{\mathbf{A}}_{k}^{v} \times\{0\}$ of $\hat{\mathbf{A}}_{k}^{d}$.

3. There exists $\varphi$ in $\mathrm{G}_{f o r}$ such that $\varphi^{*}(\hat{\mathbf{V}})$ is the formal subscheme $\hat{\mathbf{A}}_{k}^{v} \times\{0\}$ of $\hat{\mathbf{A}}_{k}^{d}$. Similarly, the following two conditions are equivalent:

1. $\hat{\mathrm{V}}$ is the formal scheme attached to some germ at 0 of smooth analytic subspace of dimension $v$ of the $d$-dimensional affine space over $k$.

2. There exists $\varphi$ in $\mathrm{G}_{\omega}$ such that $\varphi^{*}(\hat{\mathrm{V}})$ is the formal subscheme $\hat{\mathbf{A}}_{k}^{v} \times\{0\}$ of $\hat{\mathbf{A}}_{k}^{d}$. When they are satisfied, we shall say that the formal germ $\hat{\mathrm{V}}$ is analytic and smooth.

These observations lead to define the size of a smooth formal subscheme $\hat{\mathrm{V}}$ of dimension $v$ of $\hat{\mathbf{A}}_{k}^{d}$ as the supremum $\mathbf{R}(\hat{\mathbf{V}})$ in $[0,1]$ of the real numbers $\left.\left.r \in\right] 0,1\right]$ for which there exists $\varphi$ in $\mathrm{G}_{\omega}(r)$ such that $\varphi^{*}(\hat{\mathbf{V}})$ is the formal subscheme $\hat{\mathbf{A}}_{k}^{v} \times\{0\}$ of $\hat{\mathbf{A}}_{k}^{d}$. It is positive if and only if $\hat{\mathrm{V}}$ is analytic. Moreover, it satisfies the following invariance properties, the proof of which is a simple exercise:

Lemma 3.1. - Let $\hat{\mathrm{V}}$ be a smooth formal subscheme of $\hat{\mathbf{A}}_{k}^{d}$.

1) For any $f \in \operatorname{Aut} \hat{\mathbf{A}}_{\mathscr{O}}^{d}:=\mathrm{G}_{\omega}(1)$, the sizes of $\hat{\mathrm{V}}$ and $f^{*}(\hat{\mathrm{V}})$ coincide.

2) For any integer $d^{\prime}>d$, the sizes of the formal subschemes $\hat{\mathrm{V}}$ in $\hat{\mathbf{A}}_{k}^{d}$ and $\hat{\mathrm{V}} \times\{0\}^{d^{\prime}-d}$ in $\hat{\mathbf{A}}_{k}^{d^{\prime}}$ coincide.

Size of a smooth formal subscheme of a general k-scheme. - More generally, if $\mathscr{C}$ is an $\mathscr{O}$-scheme equipped with a section $\mathscr{P} \in \mathscr{C}(\mathscr{O})$ and if $\hat{\mathrm{V}}$ is a smooth formal 
subscheme of the formal completion $\hat{\mathrm{X}}_{\mathrm{P}}$ of $\mathrm{X}:=\mathscr{C}_{k}$ at $\mathrm{P}:=\mathscr{P}_{k}$, then the size $\mathrm{R}_{\mathscr{K}}(\hat{\mathrm{V}})$ of $\hat{\mathrm{V}}$ with respect to the model $\mathscr{C}$ of $\mathrm{X}$ may be defined as the size of $i(\hat{\mathrm{V}})$, where $i: \mathrm{U} \hookrightarrow \mathbf{A}_{\mathscr{O}}^{d}$ is an embedding of some open neighbourhood $\mathrm{U}$ in $\mathscr{C}$ of the section $\mathscr{P}$ into an affine space of large enough dimension $d$, which moreover maps $\mathscr{P}$ to the origin $0 \in \mathbf{A}_{\mathscr{O}}^{d}(\mathscr{O})$. Lemma 3.1 shows that this definition is independent of the choices of $\mathrm{U}$, $d$, and $i$, and extends the previous one.

For instance, if $\mathscr{T}$ is a subscheme of $\mathscr{C}$ containing the section $\mathscr{P}$ such that the structural morphism $\mathscr{V} \rightarrow \operatorname{Spec} \mathscr{O}$ is smooth along $\mathrm{P}$ and if $\hat{\mathrm{V}}$ is the formal completion at $\mathrm{P}_{k}$ of $\mathscr{T}_{k}$, then $\mathrm{R}_{\mathscr{X}}(\hat{\mathrm{V}})=1$. Similarly, when $\mathscr{C}$ is smooth, local étale coordinates may be used to evaluate the size of a smooth formal subscheme of $\mathrm{X}$, as shown by the following straightforward consequence of Lemma 3.1:

Lemma 3.2. - With the notation above, if $\mathscr{C}$ is smooth along $\mathscr{P}$ and if $\left(x_{1}, \ldots, x_{d}\right)$ are regular functions on some open neighbourhood $\Omega$ of the image of $\mathscr{P}$ such that

$$
x:=\left(x_{1}, \ldots, x_{d}\right): \Omega \rightarrow \mathbf{A}_{\mathscr{O}}^{d}
$$

is étale and maps $\mathscr{P}$ to $0 \in \mathbf{A}_{\mathscr{O}}^{d}(\mathcal{O})$, then for any smooth formal subscheme $\hat{\mathrm{V}}$ of $\hat{\mathrm{X}}_{\mathrm{P}}$, the size $\mathbf{R}_{\mathscr{X}}(\hat{\mathrm{V}})$ coincides with the size of the image $\hat{x}(\hat{\mathrm{V}})$ of $\hat{\mathrm{V}}$ in $\hat{\mathbf{A}}_{k}^{d}$ by the formal isomorphism $\hat{x}: \hat{\mathrm{X}}_{\mathrm{P}} \rightarrow \hat{\mathbf{A}}_{k}^{d}$ defined by $x$.

Size and norms of evaluation maps. - Let us keep the notation of the preceding paragraphs. The tangent space $\mathrm{T}_{\mathrm{P}} \hat{\mathrm{V}}$ of $\hat{\mathrm{V}}$ at $\mathrm{P}$ and its dual $\check{\mathrm{T}}_{\mathrm{P}} \hat{\mathrm{V}}$ are endowed with natural $\mathscr{O}$-structures, dual of each other: the $\mathscr{O}$-structure on $\check{\mathrm{T}}_{\mathrm{P}} \mathrm{V}$ may be defined by the $\mathscr{O}$-submodule image of the composite map

$$
\mathscr{P}^{*} \Omega_{\mathscr{K} / \mathrm{R}}^{1} \rightarrow\left(\mathscr{P}^{*} \Omega_{\mathscr{K} / \mathrm{R}}^{1}\right)_{k} \simeq \Omega_{\mathrm{X} / k, \mathrm{P}}^{1} \rightarrow \check{\mathrm{T}}_{\mathrm{P}} \hat{\mathrm{V}}
$$

equivalently, the differential of the embedding $i_{k}: \mathrm{U}_{k} \hookrightarrow \mathbf{A}_{k}^{d}$ defines an injective map

$$
\mathrm{D} i_{k}: \mathrm{T}_{\mathrm{P}} \hat{\mathrm{V}} \hookrightarrow \mathrm{T}_{0} \mathbf{A}_{k}^{d} \simeq k^{d}
$$

which maps the $\mathscr{O}$-integral elements of $\mathrm{T}_{\mathrm{P}} \hat{\mathrm{V}}$ onto $\mathcal{O}^{d} \cap i_{k}\left(\mathrm{~T}_{\mathrm{P}} \hat{\mathrm{V}}\right)$. For any $i \in \mathbf{N}$, we

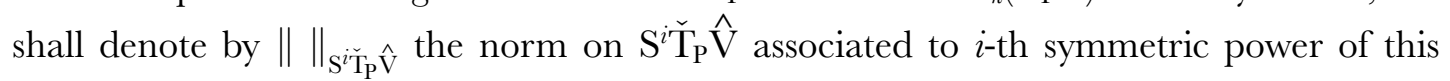
(C)-structure.

In the sequel, sizes of smooth formal subschemes will be used to bound norms of evaluation maps, by means of the following lemma which directly follows from the preceding definitions. 
Lemma 3.3. - Let us keep the above notation, and let $\Omega$ be an open subscheme of $\mathscr{C}$ containing the section $\mathscr{P}$. For any non-negative integer $i$ and any regular function $s$ on $\Omega$ such that $s_{\mathrm{K} \mid \hat{\mathrm{V}}}$ vanishes at order $i-1$ at $\mathrm{P}$, the jet $j^{i} s$ of order $i$ of $s$ on $\hat{\mathrm{V}}-$ seen as an element of $\mathrm{S}^{i \Upsilon_{\mathrm{T}} \mathrm{V}}$ - satisfies the following bound:

$$
\left\|j^{i} s\right\|_{\mathrm{S}^{i} \breve{\mathrm{T}}_{\mathrm{P}} \hat{\mathrm{V}}} \leqslant \mathrm{R} \mathscr{C}(\hat{\mathrm{V}})^{-i}
$$

\subsubsection{Global theory}

Let $\mathrm{K}$ be a number field and $\mathscr{O}_{\mathrm{K}}$ its ring of integers. If $\mathscr{C}$ is an $\mathscr{O}_{\mathrm{K}}$-scheme equipped with an integral point $\mathscr{P} \in \mathscr{X}\left(\mathscr{O}_{\mathrm{K}}\right)$ and if $\hat{\mathrm{V}}$ is a smooth formal subscheme of the formal completion $\hat{\mathrm{X}}_{\mathrm{P}_{\mathrm{K}}}$ of $\mathrm{X}:=\mathscr{C}_{\mathrm{K}}$ at $\mathrm{P}:=\mathscr{P}_{\mathrm{K}}$, we may apply the previous constructions at every finite place $\mathbf{p} \in \mathrm{V}_{f}(\mathrm{~K})$. Namely, if $\mathbf{p}$ is any non-zero prime ideal of $\mathscr{O}_{\mathrm{K}}$, we may consider the $\mathbf{p}$-adic completion $\mathrm{K}_{\mathrm{p}}\left(\right.$ resp. $\left.\mathscr{O}_{\mathbf{p}}\right)$ of $\mathrm{K}$ (resp. of $\mathscr{C}_{\mathrm{K}}$ ), which is equipped with the $\mathbf{p}$-adic absolute value ||$_{\mathbf{p}}$, and therefore define the size $\mathrm{R}_{\mathscr{X}, \mathbf{p}}(\hat{\mathrm{V}}):=\mathrm{R}_{\mathscr{C}_{\mathscr{p}}}(\hat{\mathrm{V}})$ by means of the previous construction with $k=\mathrm{K}_{\mathbf{p}}$ and ||$=||_{\mathbf{p}}$.

Finally, if $\mathrm{X}$ is a scheme over $\mathrm{K}$, say quasi-projective, equipped with a rational point $\mathrm{P}$, we may find an $\mathscr{O}_{\mathrm{K}}$-scheme $\mathscr{C}$ which is a model of $\mathrm{X}$ such that $\mathrm{P}$ extends to an integral point $\mathscr{P}$ in $\mathscr{C}\left(\mathscr{O}_{\mathrm{K}}\right)$, and, for any smooth formal subscheme $\hat{\mathrm{V}}$ of the formal completion $\hat{\mathrm{X}}_{\mathrm{P}}$, we may consider the family $\left(\mathrm{R}_{\mathscr{C}, \mathbf{p}}(\hat{\mathrm{V}})\right)_{\mathbf{p} \in \mathrm{V}_{f}(\mathrm{~K})}$ in $[0,1]^{\mathrm{V}_{f}(\mathrm{~K})}$. If $\mathscr{C}^{\prime}$ is another such model of $\mathrm{X}$, then $\mathrm{R}_{\mathscr{X}}, \mathbf{p}(\hat{\mathrm{V}})$ and $\mathrm{R}_{\mathscr{X}}^{\prime}, \mathbf{p}(\hat{\mathrm{V}})$ coincide for almost every $\mathbf{p}$ in $\mathrm{V}_{f}(\mathrm{~K})$; moreover, for every $\mathbf{p}$ in $\mathrm{V}_{f}(\mathrm{~K}), \mathbf{R}_{\mathscr{X}}, \mathbf{p}(\hat{\mathrm{V}})$ is positive if and only if $\hat{\mathrm{V}}_{\mathrm{K}_{\mathbf{p}}}$ is analytic, and therefore if and only if $\mathrm{R}_{\mathscr{X}}^{\prime}, \mathbf{p}(\hat{\mathrm{V}})$ is positive.

By replacing $\mathrm{X}$ by an affine open neighbourhood of $\mathrm{P}$, these definitions extend to the situation where $\mathrm{X}$ is an arbitrary $\mathrm{K}$-scheme of finite type.

I would like to end this section with a word of caution: when $\hat{V}$ is the graph in $\hat{\mathbf{A}}_{\mathrm{K}}^{d}$ of some formal series $f \in \mathrm{K}\left[\left[\mathrm{X}_{1}, \ldots, \mathrm{X}_{d-1}\right]\right]$ vanishing at 0 , the sizes of $\hat{\mathrm{V}}$ are in general strictly smaller than the respective $\mathbf{p}$-adic radii of convergence (consider for instance $\log (1+\mathrm{X}) \in \mathbf{Q}[\mathrm{X}]])$.

\subsection{Main Theorem}

Theorem 3.4. - Let $\mathrm{X}$ be an algebraic variety over a number field $\mathrm{K}, \mathrm{P}$ a point in $\mathrm{X}(\mathrm{K})$, and $\hat{\mathrm{V}}$ a smooth formal subscheme (over $\mathrm{K}$ ) of the completion $\hat{\mathrm{X}}_{\mathrm{P}}$ of $\mathrm{X}$ at $\mathrm{P}$.

Let us denote $\left(\mathrm{R}_{\mathbf{p}}\right)_{\mathbf{p} \in \mathrm{V}_{f}(\mathrm{~K})}$ the family of sizes of $\hat{\mathrm{V}}$ with respect to some integral model of $\mathrm{X}$ over $\mathscr{O}_{\mathrm{K}}$, and suppose that the following conditions are satisfied: 
i) For any $\mathbf{p} \in \mathrm{V}_{f}(\mathrm{~K}), \mathrm{R}_{\mathbf{p}}$ is positive and

$$
\sum_{\mathbf{p} \in \mathrm{V}_{f}(\mathrm{~K})} \log \mathrm{R}_{\mathbf{p}}^{-1}<\infty .
$$

ii) For any imbedding $\sigma: \mathrm{K} \hookrightarrow \mathbf{G}$, the smooth formal subscheme $\hat{\mathrm{V}}_{\sigma}$ of $\hat{\mathrm{X}}_{\sigma, \mathrm{P}_{\sigma}}$ is the formal subscheme associated to some germ $\mathrm{V}_{\sigma}^{a n}$ of analytic submanifold of $\mathrm{X}_{\sigma}(\mathbf{G})$ at $\mathrm{P}_{\sigma}$.

iii) There exists an embedding $\sigma_{0}: \mathrm{K} \hookrightarrow \mathbf{G}$, a complex manifold $\mathrm{M}$ satisfying the Liouville property, a point $\mathrm{O}$ of $\mathrm{M}$, and a holomorphic map $f: \mathrm{M} \rightarrow \mathrm{X}_{\sigma_{0}}(\mathbf{G})$, which sends $\mathrm{O}$ to $\mathrm{P}_{\sigma_{0}}$ and maps the germ of $\mathrm{M}$ at $\mathrm{O}$ biholomorphically onto the germ $\mathrm{V}_{\mathbf{\sigma}_{0}}^{a n}$.

Then there exists an algebraic subvariety $\mathrm{Y}$ of $\mathrm{X}$ of the same dimension as $\hat{\mathrm{V}}$ such that $\mathrm{P} \in \mathrm{Y}(\mathrm{K})$ and $\hat{\mathrm{V}} \subset \hat{\mathrm{Y}}_{\mathrm{P}}$ (equivalently, $\hat{\mathrm{V}}$ is a branch of $\mathrm{Y}$ at $\mathrm{P}$ ).

This algebraization statement is in the same spirit as results of D. V. and G. V. Chudnovsky ([CC85a], [CC85b]), and André ([And89], Chapter VIII, and [And99]). However, these authors prove algebraicity of functions only. Moreover, in loc. cit., the $p$-adic conditions which play the role of condition i) above take a stronger or more complicated form, and condition iii) is replaced by conditions involving uniformization by large enough polydisks or by an affine space, possibly satisfying growth conditions.

Observe that condition i) in Theorem 3.4 does not depend on the choice of the model used to define $\left(\mathbf{R}_{\mathbf{p}}\right)_{\mathbf{p} \in \mathrm{V}_{f}(\mathrm{~K})}$, and may be rephrased as

$$
\prod_{\mathbf{p} \in \mathrm{V}_{f}(\mathrm{~K})} \mathrm{R}_{\mathbf{p}}>0 .
$$

Similar conditions on radii of convergence arise naturally in the theory of G-functions and are called Bombieri conditions (see [Bom81] and [DGS94]).

Also observe that the condition on the germ of analytic manifold $\mathrm{V}_{\sigma_{0}}^{a n}$ in the complex algebraic variety $\mathrm{X}_{\sigma_{0}}$ expressed by iii) is local in the Zariski topology of $\mathrm{X}_{\sigma_{0}}$. Namely, if $\mathrm{U}$ is any Zariski open neighbourhood of $\mathrm{P}_{\sigma_{0}}$ in $\mathrm{X}_{\sigma_{0}}$, this condition holds if and only if the analogous condition where $\mathrm{U}$ is substituted to $\mathrm{X}_{\sigma_{0}}$ holds. This follows from the simple facts concerning the Liouville property recalled in 2.1.2.

Finally, the conclusion of Theorem 3.4 is equivalent to the existence of a smooth algebraic variety $\mathrm{Y}^{\prime}$ over $\mathrm{K}$, of a point $\mathrm{P}^{\prime} \in \mathrm{Y}^{\prime}(\mathrm{K})$ and of a morphism of $\mathrm{K}$-varieties $v: \mathrm{Y}^{\prime} \rightarrow \mathrm{X}$ which sends $\mathrm{P}^{\prime}$ to $\mathrm{P}$ and induces a formal isomorphism from $\hat{\mathrm{Y}}_{\mathrm{P}^{\prime}}^{\prime}$ onto $\hat{V}$ (consider the normalization of $\mathrm{Y}$ and use the compatibility of normalization and completion). This shows that conditions i), ii), and iii) in Theorem 3.4 are not only sufficient, but also necessary for the validity of its conclusion. Actually, when this conclusion holds, almost all the sizes $\mathbf{R}_{\mathbf{p}}$ are equal to 1 , and $\mathrm{M}$ and $f$ may be chosen algebraic. 


\subsection{A geometric analogue}

After the submission of the first version of this paper, the author realized that a greatly simplified version of the arguments used to establish the arithmetic algebraicity criterion in Theorem 3.4 leads to the following geometric counterpart:

Theorem 3.5. - Let $\mathrm{X}$ be a quasiprojective variety over a field $k$, let $\mathrm{V}_{0}$ be a smooth connected projective subvariety of $\mathrm{X}$, of dimension $\geqslant 1$, and let $\hat{\mathrm{V}}$ be a smooth formal subscheme of dimension $d$ of the formal completion $\hat{\mathrm{X}}_{\mathrm{V}_{0}}$ of $\mathrm{X}$ along $\mathrm{V}_{0}$, admitting $\mathrm{V}_{0}$ as scheme of definition. If the normal bundle $\mathrm{N}_{\mathrm{V}_{0}} \hat{\mathrm{V}}$ of $\mathrm{V}_{0}$ in $\hat{\mathrm{V}}$ is big (e.g., if it is ample), then $\hat{\mathrm{V}}$ is algebraic, namely there exists a closed algebraic subvariety $\mathrm{Y}$ of dimension $d$ in $\mathrm{X}$, such that $\mathrm{V}_{0} \subset \mathrm{Y}$ and $\hat{\mathrm{V}} \subset \hat{\mathrm{Y}}_{\mathrm{V}_{0}}$ (equivalently, $\hat{\mathrm{V}}$ is a branch of $\mathrm{Y}$ along $\mathrm{V}_{0}$ ).

This statement immediately implies the following function field version of Theorem 3.4:

Theorem 3.6. - Let $\mathrm{C}$ be a smooth, projective, geometrically connected curve over a field $k$, and let $\mathrm{K}:=k(\mathrm{C})$ be its field of rational functions. Let $\mathrm{X}$ be a quasiprojective algebraic variety over $\mathrm{K}, \mathrm{P}$ a point in $\mathrm{X}(\mathrm{K})$, and $\hat{\mathrm{V}}$ a smooth formal subscheme (over $\mathrm{K}$ ) of the completion $\hat{\mathrm{X}}_{\mathrm{P}}$ of $\mathrm{X}$ at $\mathrm{P}$.

Let us consider a model $f: \mathscr{C} \rightarrow \mathrm{C}$ of $\mathrm{X}$ over $\mathrm{C}$ such that $\mathscr{C}$ is quasiprojective over $k$ and $\mathrm{P}$ extends to a section $\mathscr{P}$ of $f$ over $\mathrm{C}$, and suppose that the following conditions are satisfied:

i) The smooth formal subscheme of $\hat{\mathrm{X}}_{\mathrm{P}}$ extends to a smooth formal subscheme $\hat{\mathscr{V}}$ of the completion of $\mathscr{C}$ along $\mathscr{P}$.

ii) The normal bundle $\mathrm{N}_{\mathscr{P}} \hat{\mathscr{V}}$ of $\hat{\mathscr{V}}$ along $\mathscr{P}$ is ample.

Then $\hat{\mathrm{V}}$ is a branch of some algebraic subvariety of $\mathrm{X}$ containing $\mathrm{P}$.

In the arithmetic Theorem 3.4, conditions i) and ii) may be seen as smoothness conditions, analogous to condition i) in Theorem 3.6, while the Liouville condition iii) entails a strong form of arithmetic positivity for the normal bundle of $\mathrm{P}$ in $\hat{\mathrm{V}}$.

Apparently, Theorem 3.5 does not appear explicitly in the published literature, in spite of the simplicity of its proof and the striking applications it admits. It has also been recently noticed by Bogomolov and McQuillan, who use it in their study of rational curves along algebraic foliations, to simplify and extend the work of Miyaoka on algebraic foliations already alluded to above (cf. [BMQ01] and [Miy87]).

Actually, Theorem 3.5 is a simple consequence of some results in [Har68], used by Hartshorne to prove that, if $\mathrm{Y}$ is a complete l.c.i. subvariety of a smooth variety $\mathrm{X}$ over a field $k$, and if the normal bundle $\mathrm{N}_{\mathrm{Y}} \mathrm{X}$ of $\mathrm{Y}$ in $\mathrm{X}$ is ample, then $\mathrm{Y}$ is $G 2$ in $\mathrm{X}$ (in other 
words, the field $k\left(\hat{\mathrm{X}}_{\mathrm{Y}}\right)$ of meromorphic ${ }^{(10)}$ functions on the formal completion $\hat{\mathrm{X}}_{\mathrm{Y}}$ of $\mathrm{X}$ along $\mathrm{Y}$ is a finite degree extension of the field $k(\mathrm{X})$ of rational functions on $\mathrm{X}$; see also [Hir68] and [HM68] for related results).

A key point in the derivation of Hartshorne's G2 Theorem is the following fact:

Theorem 3.7 ([Har68], Theorem 6.7). — Let be a smooth and proper formal scheme of dimension d over a field $k$ and let $\mathrm{V}_{0}$ be a subscheme of definition of $\hat{\mathrm{V}}$. Assume that $\mathrm{V}_{0}$ is connected, of dimension $\geqslant 1$, and locally a complete intersection in $\hat{\mathrm{V}}$. If the normal bundle $\mathrm{N}_{\mathrm{V}_{0}} \hat{\mathrm{V}}$ of $\mathrm{V}_{0}$ in $\hat{\mathrm{V}}$ is ample, then the transcendence degree of the field $\mathrm{K}(\hat{\mathrm{V}})$ of meromorphic functions on $\hat{\mathrm{V}}$ is at most $d$.

This statement implies the following variant of Theorem 3.5:

Corollary 3.8. - Let $\mathrm{X}$ be a scheme of finite type over a field $k$, let $\mathrm{V}_{0}$ be a subscheme of $\mathrm{X}$, proper over $k$, connected and of dimension $\geqslant 1$, and let $\hat{\mathrm{V}}$ be a smooth formal subscheme of dimension d of the formal completion $\hat{\mathrm{X}}_{\mathrm{V}_{0}}$ of $\mathrm{X}$ along $\mathrm{V}_{0}$, admitting $\mathrm{V}_{0}$ as scheme of definition. If $\mathrm{V}_{0}$ is locally a complete intersection and if the normal bundle $\mathrm{N}_{\mathrm{V}_{0}} \hat{\mathrm{V}}$ of $\mathrm{V}_{0}$ in $\hat{\mathrm{V}}$ is ample, then $\hat{\mathrm{V}}$ is algebraic.

Indeed, we may consider the Zariski closure $\mathrm{Y}$ of $\hat{\mathrm{V}}$ in $\mathrm{X}$, namely the smallest closed subscheme of $\mathrm{X}$ containing $\hat{\mathrm{V}}$. It is an integral subscheme of $\mathrm{X}$ such that $\mathrm{V}_{0} \subset \mathrm{Y}$ and $\hat{\mathrm{V}} \subset \hat{\mathrm{Y}}_{\mathrm{V}_{0}}$. Moreover, the field $\mathrm{K}(\mathrm{Y})$ of rational functions on $\mathrm{Y}$ may be identified with a subfield of $\mathrm{K}(\hat{\mathrm{V}})$, and therefore Hartshorne's Theorem 3.7 shows that the dimensions of $\hat{\mathrm{V}}$ and $\mathrm{Y}$ coincide.

The proof of Theorem 3.7 relies on the following estimate, where $\mathrm{L}$ denotes a line bundle over $\hat{\mathrm{V}}$ and $\mathrm{D}$ an integer which goes to $+\infty$ :

$$
\operatorname{rk~} \mathrm{H}^{0}\left(\hat{\mathrm{V}}, \mathrm{L}^{\otimes \mathrm{D}}\right)=\mathrm{O}\left(\mathrm{D}^{\operatorname{dim} \hat{V}}\right)
$$

(see [Har68], §6). Such an upper bound also directly implies Theorem 3.5. Indeed, to prove it, we may assume that $\mathrm{X}$ is projective and apply (3.2) with $\mathrm{L}$ an ample line bundle on $\mathrm{X}$. If $\mathrm{Y}$ denotes the Zariski closure of $\hat{V}$ in $\mathrm{X}$, then $\mathrm{H}^{0}\left(\hat{\mathrm{V}}, \mathrm{L}^{\otimes D}\right)$ injects into $\mathrm{H}^{0}\left(\mathrm{Y}, \mathrm{L}^{\otimes \mathrm{D}}\right)$ and the characterization of the dimension of $\mathrm{Y}$ as the degree of its Hilbert polynomial shows that $\operatorname{dim} \mathrm{Y}$ is not greater than (hence equal to) $\operatorname{dim} \hat{\mathrm{V}}$.

To prove (3.2) and Theorem 3.5, one filters $\mathrm{H}^{0}\left(\hat{\mathrm{V}}, \mathrm{L}^{\otimes \mathrm{D}}\right)$ by the order of vanishing along $\mathrm{V}_{0}$; this leads to the estimate, where $\mathrm{N}:=\mathrm{N}_{\mathrm{V}_{0}} \hat{\mathrm{V}}$ :

$$
\mathrm{rk} \mathrm{H}^{0}\left(\hat{\mathrm{V}}, \mathrm{L}^{\otimes \mathrm{D}}\right) \leqslant \sum_{i \geqslant 0} \mathrm{rkH} \mathrm{H}^{0}\left(\mathrm{~V}_{0}, \mathrm{~S}^{i} \check{\mathrm{N}} \otimes \mathrm{L}^{\otimes \mathrm{D}}\right) .
$$

(10) Also called formal-rational; $c f$. [HM68]. 
Finally, $\mathrm{rk} \mathrm{H}^{0}\left(\mathrm{~V}_{0}, \mathrm{~S}^{i} \mathrm{~N} \otimes \mathrm{L}^{\otimes \mathrm{D}}\right)$ grows at most like $(i+\mathrm{D})^{\operatorname{dim} \mathrm{V}_{0}+\mathrm{rk} F-1}$, and vanishes when $i / \mathrm{D}$ is large enough since $\mathrm{N}_{\mathrm{V}_{0}} \hat{\mathrm{V}}$ is big. Since

$$
\operatorname{dim} \hat{\mathrm{V}}=\operatorname{dim} \mathrm{V}_{0}+\mathrm{rk} \mathrm{F},
$$

this entails (3.2) (see also [Har68] §6, [BMQ01], 2.1.1, and [CB01], 4.7).

\subsection{Vanishing of p-curvatures and $\mathbf{p}$-adic sizes of formal leaves}

In this section, we derive the algebraicity criterion for leaves of foliations stated in Theorem 2.1 from the algebraicity criterion for formal germs in Theorem 3.4. To achieve this, we apply the latter to the formal germ $\hat{\mathrm{V}}$ of leaf through $\mathrm{P}$ defined by the involutive bundle $\mathrm{F}$ (see 3.4.1, infra). This formal germ $\hat{\mathrm{V}}$ is smooth; moreover, for any $\mathbf{p} \in \mathrm{V}_{f}(\mathrm{~K})$, the formal subscheme $\hat{\mathrm{V}}_{\mathrm{K}_{\mathbf{p}}}$ of $\hat{\mathrm{X}}_{\mathrm{P}, \mathrm{K}_{\mathbf{p}}}$ is $\mathrm{K}_{\mathbf{p}}$-analytic - in other words, its size $R_{\mathbf{p}}$ with respect to any model is positive - and for any embedding $\sigma: \mathrm{K} \hookrightarrow \mathbf{C}$, the formal subscheme $\hat{\mathrm{V}}_{\sigma}$ of $\hat{\mathrm{X}}_{\sigma, \mathrm{P}_{\sigma}}$ is $\mathbf{C}$-analytic. The Liouville condition (Theorem 3.4 , iii) holds by hypothesis, and the Bombieri condition (3.1) will follow from the assumption of $p$-integrability for almost every reduction. Indeed, it yields the following upper bound for almost every $\mathbf{p}$, of residual characteristic $p$ :

$$
\log \mathrm{R}_{\mathbf{p}}^{-1} \leqslant 3\left[\mathrm{~K}_{\mathbf{p}}: \mathbf{Q}_{p}\right] \frac{\log p}{p^{2}} .
$$

That type of estimate is well known for the radii of convergence of solutions of linear differential systems (see for instance [DGS94], pp. 95-96); the foliation case we consider here is similar (see Proposition 3.9, infra).

Observe that, with no integrability condition, the sizes $R_{p}$ are "much smaller": one gets that, for almost every $\mathbf{p}$,

$$
\log \mathrm{R}_{\mathbf{p}}^{-1} \leqslant\left[\mathrm{~K}_{\mathbf{p}}: \mathbf{Q}_{p}\right] \frac{\log p}{p-1}
$$

(see Proposition 3.9, infra, and its proof), and basically this bound is optimal, as examplified, for instance, by the graph of the usual exponential. These estimates also show that, in Theorem 2.1 and its consequences Theorems 2.3 and 2.9, the $p$-integrability conditions for almost every $\mathbf{p}$ could be weakened to the $p$-integrability for all $\mathbf{p} \in \mathrm{V}_{f}(\mathrm{~K})$ not dividing the elements in a set $\mathrm{S}$ of rational primes which has "density zero" in the sense that

$$
\sum_{p \in \mathrm{S}} \frac{\log p}{p}<\infty
$$




\subsubsection{Formal flows and formal leaves}

With the notation of Theorem 2.1, let us choose regular functions $x_{1}, \ldots, x_{d}$ on some open Zariski neighbourhood $\mathrm{U}$ of $\mathrm{P}$ in $\mathrm{X}$ such that $\left(x_{1}, \ldots, x_{d}\right): \mathrm{U} \rightarrow \mathbf{A}_{\mathrm{K}}^{d}$ is étale and sends $\mathrm{P}$ to 0 . Then the coherent sheaf of regular sections of $\mathrm{T}_{\mathrm{X}}$ is free on $\mathrm{U}$ with basis $\left(\partial / \partial x_{1}, \ldots, \partial / \partial x_{n}\right)$. Moreover, after possibly permuting the $x_{i}$ 's and shrinking $\mathrm{U}$, we may assume that, for any integer $i$ such that $1 \leqslant i \leqslant f:=\operatorname{rk} \mathrm{F}$, there exists a unique regular section $v_{i}$ of $\mathrm{F}$ over $\mathrm{U}$ which may be written

$$
v^{i}=\frac{\partial}{\partial x_{i}}+\sum_{k=f+1}^{d} a_{k}^{i} \frac{\partial}{\partial x_{k}}
$$

for some $a_{k}^{i} \in \mathcal{O}(\mathbf{U})$. Then $\left(v^{1}, \ldots, v^{f}\right)$ is a basis of the sheaf of regular sections of $\mathbf{F}$ over $\mathrm{U}$, and the involutivity of $\mathrm{F}$ shows that the Lie brackets $\left[v^{i}, v^{j}\right]$ vanish over $\mathrm{U}$. The $p$-integrability hypothesis on $\mathrm{F}$ also shows that, for almost every $\mathbf{p} \in \mathrm{V}_{f}(\mathrm{~K})$, the reductions $v_{\mathbf{F}_{\mathbf{p}}}^{i}$ of these sections have vanishing $p$-th powers, if $p$ denotes, as usual, the characteristic of $\mathbf{F}_{\mathbf{p}}$.

Consider now the formal flow

$$
\psi: \hat{\mathbf{A}}_{K}^{f} \hat{\times} \hat{\mathrm{X}}_{\mathrm{P}} \rightarrow \hat{\mathrm{X}}_{\mathrm{P}}
$$

attached to the commuting vector fields $v^{1}, \ldots, v^{f}$ on $\hat{\mathrm{X}}_{\mathrm{P}}$. Let us briefly recall how it is defined.

By means of the local coordinates $x_{1}, \ldots, x_{d}$, we may identify $\hat{\mathrm{X}}_{\mathrm{P}}$ with the formal spectrum of $\mathrm{K}\left[\left[x_{1}, \ldots, x_{d}\right]\right]$, the vector fields $v^{1}, \ldots, v^{f}$ with elements of $\mathrm{K}\left[\left[x_{1}, \ldots, x_{d}\right]\right]^{d}$, and $\psi$ with an element of $\mathrm{K}\left[\left[t_{1}, \ldots, t_{f}, x_{1}, \ldots, x_{d}\right]\right]^{d}$. Then $\psi$ is characterized by the following two properties: firstly,

$$
\psi\left(0, \ldots, 0, x_{1}, \ldots, x_{d}\right)=X:=\left(\begin{array}{c}
x_{1} \\
\vdots \\
x_{d}
\end{array}\right)
$$

secondly, for any $i \in\{1, \ldots, f\}$,

$$
\frac{\partial \psi}{\partial t_{i}}\left(t_{1}, \ldots, t_{f}, x_{1}, \ldots, x_{d}\right)=v^{i}\left(\psi\left(t_{1}, \ldots, t_{f}, x_{1}, \ldots, x_{d}\right)\right) .
$$

For any $i \in\{1, \ldots, f\}$, let us denote by $\mathrm{D}_{i}$ the derivation defined by $v^{i}$ on $\mathrm{K}\left[\left[x_{1}, \ldots, x_{d}\right]\right]$ and on $\mathrm{K}\left[\left[x_{1}, \ldots, x_{d}\right]\right]^{d}$ (acting component-wise). Then the expansion of $\psi$ in powers of $t_{1}, \ldots, t_{d}$ is given by the following "Taylor formula", which could have 
been used as definition of $\psi$ :

$$
\psi\left(t_{1}, \ldots, t_{f}, x_{1}, \ldots, x_{d}\right)=e^{\sum_{i=1}^{f} t_{i} \mathrm{D}_{i}} \mathrm{X}:=\sum_{\mathrm{I} \in \mathbf{N}^{f}} \frac{t^{\mathrm{I}}}{\mathrm{I} !} \mathrm{D}^{\mathrm{I}} \mathrm{X}
$$

where, for any $\mathrm{I}=\left(i_{1}, \ldots, i_{f}\right) \in \mathbf{N}^{f}, \mathrm{D}^{\mathrm{I}}:=\mathrm{D}_{1}^{i_{1}} \ldots \mathrm{D}_{f}^{i_{f}}, t^{\mathrm{I}}:=t_{1}^{i_{1}} \ldots t_{f}^{i_{f}}$, and $\mathrm{I} !:=i_{1} ! \ldots i_{f} !$. Observe that the formal series $\psi$ indeed defines a "formal action" of $\hat{\mathbf{G}}_{a, \mathrm{~K}}^{f} \simeq \hat{\mathbf{A}}_{\mathrm{K}}^{f}$ on $\hat{\mathrm{X}}_{\mathrm{P}} \simeq \operatorname{Specf} \mathrm{K}\left[\left[x_{1}, \ldots, x_{d}\right]\right]$, since it satisfies $\psi(0, x)=X$ and $\psi\left(t+t^{\prime}, x\right)=\psi\left(t^{\prime}, \psi(t, x)\right)$.

The formal leaf $\hat{\mathrm{V}}$ of $\mathrm{F}$ through $\mathrm{P}$ is the formal subscheme of $\hat{\mathrm{X}}_{\mathrm{P}}$ defined as the image of the formal embedding $\psi(:, \mathrm{P}): \hat{\mathbf{A}}_{\mathrm{K}}^{f} \rightarrow \hat{\mathrm{X}}_{\mathrm{P}}$. Expressed in terms of the local coordinates $\left(x_{1}, \ldots, x_{d}\right)$ on $\mathrm{X}$, this formal embedding is given by the formal series $\psi\left(t_{1}, \ldots, t_{f}, 0, \ldots, 0\right)$ in $\mathrm{K}\left[\left[t_{1}, \ldots, t_{f}\right]\right]^{d}$. Consider the formal isomorphism

$$
\varphi: \hat{\mathbf{A}}_{\mathrm{K}}^{d} \rightarrow \hat{\mathrm{X}}_{\mathrm{P}}
$$

which, in terms of the local coordinates $\left(x_{1}, \ldots, x_{d}\right)$, is given by the series

$$
\varphi\left(t_{1}, \ldots, t_{d}\right):=\psi\left(t_{1}, \ldots, t_{f}, 0, \ldots, 0\right)+{ }^{t}\left(0, \ldots, 0, t_{f+1}, \ldots, t_{d}\right)
$$

in $\mathrm{K}\left[\left[t_{1}, \ldots, t_{d}\right]\right]^{d}$. By construction, the restriction of $\varphi$ to $\hat{\mathbf{A}}_{\mathrm{K}}^{f} \times\{0\}$ is a formal isomorphism onto $\hat{\mathrm{V}}$, and its differential defines an isomorphism between the subbundle of the formal tangent bundle of $\hat{\mathbf{A}}_{\mathrm{K}}^{d}$ generated by $\left(\partial / \partial t_{1}, \ldots, \partial / \partial t_{f}\right)$ and the subbundle of the formal tangent bundle of $\hat{\mathrm{X}}_{\mathrm{P}}$ defined by $\mathrm{F}$. In particular, $\hat{\mathrm{V}}$ is the unique smooth formal subscheme of dimension $f$ of $\hat{\mathrm{X}}_{\mathrm{P}}$ which is a formal integral variety of $\mathrm{F}$.

Moreover, since the series defining $v_{1}, \ldots, v_{f}$ have positive $\mathbf{p}$-adic and archimedean radii of convergence, the same holds for the series (3.7) defining $\psi$ (this is well known in the archimedean case, and in the $\mathbf{p}$-adic one, follows for instance from Proposition $3.9,1)$, infra), hence for $\boldsymbol{\varphi}$. This shows that, for any $\mathbf{p}$ in $\mathrm{V}_{f}(\mathrm{~K})$ (resp. any embedding $\sigma: K \hookrightarrow \mathbf{G}$ ), the formal germ $\hat{V}_{\mathrm{K}_{\mathrm{p}}}$ (resp. $\hat{\mathrm{V}}_{\boldsymbol{\sigma}}$ ) is analytic.

Finally, the leaf of $\mathrm{F}$ through $\mathrm{P}$ is algebraic if and only if the formal leaf $\hat{\mathrm{V}}$ is a branch of a subvariety of $\mathrm{X}$ containing $\mathrm{P}$. Thanks to the algebraicity criterion in Theorem 3.4, to complete the proof of Theorem 2.1, it is enough to show that almost all the p-adic sizes of $\hat{V}$ satisfy the estimates (3.3). This will follow from Proposition 3.9 in the next subsection, together with Lemma 3.2.

\subsection{2. $\mathbf{p}$-adic estimates on formal flows}

Let $\mathbf{p}$ be any element in $\mathrm{V}_{f}(\mathrm{~K}), p$ the characteristic of $\mathbf{F}_{\mathbf{p}}$, and $\varpi$ a uniformizing element in $\mathcal{O}_{\mathbf{p}}$. 
Proposition 3.9. - Let $v^{1}, \ldots, v^{f}$ be commuting formal vector fields in $\mathcal{O}_{\mathbf{p}}\left[\left[x_{1}, \ldots, x_{d}\right]\right]^{d}$, let $\mathrm{D}_{1}, \ldots, \mathrm{D}_{f}$ be the corresponding derivations in $\bigoplus_{i=1}^{d} \mathcal{O}_{\mathbf{p}}\left[\left[x_{1}, \ldots, x_{d}\right]\right] \partial / \partial x_{i}$, and let

$$
\psi\left(t_{1}, \ldots, t_{f}, x_{1}, \ldots, x_{d}\right) \in \mathrm{K}_{\mathbf{p}}\left[\left[t_{1}, \ldots, t_{f}, x_{1}, \ldots, x_{d}\right]\right]^{d}
$$

be their formal flow, defined by the properties (3.5) and (3.6), or by the expansion (3.7).

1) The series $\psi\left(t_{1}, \ldots, t_{f}, x_{1}, \ldots, x_{d}\right)$ converges on the polydisk defined by

$$
\left|t_{j}\right|_{\mathbf{p}}<|p|_{\mathbf{p}}^{\frac{1}{p-1}}=p^{-\left[\mathrm{K}_{\mathbf{p}}: \mathbf{Q}_{p}\right] /(p-1)}, \quad 1 \leqslant j \leqslant f
$$

and

$$
\left|x_{k}\right|_{\mathbf{p}}<1, \quad 1 \leqslant k \leqslant d
$$

2) Suppose moreover that the reductions modulo $₫$ of the vector fields $v_{1}, \ldots, v_{f}$ have vanishing p-th powers, and that $\mathrm{K}_{\mathbf{p}}$ is absolutely unramified. Then, for any $\mathbf{I}=\left(i_{1}, \ldots, i_{f}\right)$ in $\mathbf{N}^{f} \backslash\{(0, \ldots, 0)\}$,

$$
\left|\frac{1}{\mathrm{I} !} \mathrm{D}^{\mathrm{I}}(\mathrm{X})(0)\right|_{\mathbf{p}} \leqslant|p|_{\mathbf{p}}^{-\sum_{j=1}^{f}\left(\frac{i_{j}-\mathrm{S}\left(i_{j}\right)}{p-1}-\left[\frac{i j}{p}\right]\right)}
$$

$$
\leqslant p^{\frac{3\left[\mathrm{~K}_{\mathbf{p}}: \mathbf{Q}_{p}\right]}{p^{2}}(|\mathrm{I}|-1)}
$$

where $\mathrm{S}\left(i_{j}\right)$ denotes the sum of the digits of the expansion of $i_{j}$ in the base $p$, and $|\mathrm{I}|:=\sum_{j=1}^{f} i_{j}$. In particular, the element $\varphi$ of $\mathrm{G}_{\text {for }}$ defined by (3.8) belongs to $\mathrm{G}_{\omega}(r)$ for any positive $r$ such that

$$
r \leqslant p^{-\frac{3\left[\mathrm{~K}_{\mathbf{p}}: \mathbf{Q}_{p}\right]}{p^{2}}}
$$

and the size $\mathbf{R}_{\mathbf{p}}$ of the formal subscheme $\hat{\mathrm{V}}$ of $\hat{\mathbf{A}}_{\mathrm{K}_{\mathbf{p}}}^{d}$ defined as the image of the formal embedding

$$
\psi\left(t_{1}, \ldots, t_{f}, 0, \ldots, 0\right): \hat{\mathbf{A}}_{\mathrm{K}_{\mathbf{p}}} \longrightarrow \hat{\mathbf{A}}_{\mathrm{K}_{\mathbf{p}}}^{d}
$$

satisfies

$$
\log \mathrm{R}_{\mathbf{p}}^{-1} \leqslant 3\left[\mathrm{~K}_{\mathbf{p}}: \mathbf{Q}_{p}\right] \frac{\log p}{p^{2}} .
$$

Proof. — To prove 1), observe that, for any $\mathrm{I}=\left(i_{1}, \ldots, i_{f}\right) \in \mathbf{N}^{f}$,

$$
\mathrm{D}^{\mathrm{I}}(\mathrm{X}) \in \mathcal{O}_{\mathbf{p}}\left[\left[x_{1}, \ldots, x_{d}\right]\right]^{d} \text {. }
$$

Assertion 1) then follows from the fact that, for any integer $i$, the $p$-adic valuation of $i$ ! is at most $i /(p-1)$. 
To prove 2), observe that, when the reductions modulo $₫$ of $v_{1}, \ldots, v_{f}$ have vanishing $p$-th powers, then the differential operators $\mathrm{D}_{i}^{p}, i \in\{1, \ldots, f\}$, map $\mathscr{O}_{\mathbf{p}}\left[\left[x_{1}, \ldots, x_{d}\right]\right]^{d}$ to $\varpi \mathcal{O}_{\mathbf{p}}\left[\left[x_{1}, \ldots, x_{d}\right]\right]^{d}$. This shows that, for any $\mathrm{I}=\left(i_{1}, \ldots, i_{f}\right) \in \mathbf{N}^{f}$,

$$
\mathrm{D}^{\mathrm{I}}(\mathrm{X}) \in \boldsymbol{\varpi}^{\sum_{j=1}^{f}\left[\frac{i}{p}\right]} \mathscr{\sigma}_{\mathbf{p}}\left[\left[x_{1}, \ldots, x_{d}\right]\right]^{d} .
$$

Using that the $p$-adic valuation of $i_{j}$ ! is $\left(i_{j}-\mathrm{S}\left(i_{j}\right)\right) /(p-1)$, we get (3.9). The estimate (3.10) and the other assertions immediately follow.

\subsubsection{Proof of Theorem 2.2}

Let us briefly explain how to derive the sufficiency of conditions i) and ii) in Theorem 2.2 from Theorem 3.4 and the above $p$-adic estimates on formal flows.

Clearly, we may replace the field $\mathrm{K}$ by an arbitrary finite extension and therefore we may assume that $\mathrm{X}(\mathrm{K})$ is not empty. Let us choose some point $\mathrm{P}$ in $\mathrm{X}(\mathrm{K})$, and consider the completion $\hat{\mathrm{V}}$ of $\mathrm{G}^{\text {for }}(\mathrm{X}, \mathrm{F})$ at $(\mathrm{P}, \mathrm{P})$. The formal schemes $\hat{\mathrm{V}}$ and $\mathrm{G}^{\text {for }}(\mathrm{X}, \mathrm{F})$ have the same Zariski closure in $\mathrm{X} \times_{\mathrm{K}} \mathrm{X}$. Therefore, we only need to check that the formal germ $\hat{V}$ in $X \times_{K} X$ satisfies the conditions i)-iii) in Theorem 3.4. Now, the Liouville condition iii) immediately follows from the Liouville condition ii) in Theorem 2.2 and the analyticity condition ii) is clear. Finally, Proposition 3.9 shows that the sizes $R_{\mathbf{p}}$ of $\hat{V}$ are positive and again satisfy the upper bound (3.3) when the $p$-curvature vanishes. Indeed, with the notation of paragraph 3.4.1, the map $(\varphi, \varphi)$ defines a formal isomorphism from $\hat{\mathbf{A}}_{\mathrm{K}}^{2 d}$ onto $\mathrm{X} \widehat{\times} \mathrm{X}_{(\mathrm{P}, \mathrm{P})}$, the inverse of which maps $\hat{\mathrm{V}}$ onto the formal germ in $\hat{\mathbf{A}}_{\mathrm{K}}^{2 d}$ defined by the equations $t_{f+1}=u_{f+1}, \ldots, t_{d}=u_{d}$ in the formal coordinates $\left(t_{1}, \ldots, t_{d}, u_{1}, \ldots, u_{d}\right)$.

\section{Proof of the main theorem}

This section presents a proof of Theorem 3.4, along the lines discussed in the introduction.

We would like to emphasize that the main technical difficulty in establishing our algebraicity criteria comes from factorials arising with differentiations. Similar problems related to factorials and divided power algebras associated to involutive bundles over the integers are classical in the theory of G-functions (see for instance [Sie29], Erster Teil, §4, VII, and [Bom81], §1) and also occur in Miyaoka's work ([Miy87]). Similar issues have already been addressed in section 3.4 when we estimated the sizes of formal germs of leaves.

In the proof of Theorem 3.4, these difficulties with factorials are hidden behind the arithmetic estimates in Lemma 4.8 infra: a key point is that the " $i$-th jet map" $\varphi_{\mathrm{D}}^{i}$ introduced in section 4.2.2 below - which could be expressed as a $i$-tuple derivation 
divided by a factor $i$ ! - satisfies the local estimates in Lemma 4.9, 4.10, and 4.11, while the maximal slope of the hermitian vector bundle $\mathrm{S}^{i} \check{\bar{t}} \otimes \mathscr{P}^{*} \overline{\mathscr{C}}^{\otimes \mathrm{D}}$ satisfies the bound (4.21), which is linear in $i$. In this respect, it is crucial that the estimates (4.7) and (4.29) concerning symmetric powers are linear in the exponent $k$ and $i$. As a matter of fact, crude arguments in their proof would introduce a disastrous additional factor of the form $\log i$ ! (equivalent to $i \log i$ ).

\subsection{Hermitian vector bundles, slopes, and heights of linear maps}

We now discuss a few concepts and results from Arakelov geometry which will be used in the proof of Theorem 3.4. These are quite elementary, although several of them are not explicitly established in the literature, and we have tried to make this section self-contained. We refer the reader to [Szp85], [Bos96] Appendix, and [Sou97] for more extensive discussions and references concerning hermitian vector bundles in Arakelov geometry.

We will denote by $\mathrm{K}$ a number field and by $\mathscr{O}_{\mathrm{K}}$ its ring of integers.

\subsubsection{Basic definitions}

Let $\mathscr{C}$ be a separated scheme of finite type over $\operatorname{Spec} \mathbf{Z}$ such that its generic fiber $\mathscr{C}_{\mathrm{Q}}$ (or equivalently, the complex analytic space $\mathscr{C}(\mathbf{C})$ ) is smooth. In this paper, an hermitian vector bundle $\overline{\mathrm{E}}$ over $\mathscr{C}$ will be a pair $(\mathrm{E},\|\|$.$) , where \mathrm{E}$ is a locally free coherent sheaf on $\mathscr{C}$ and $\|$.$\| is a continuous hermitian metric on the associated analytic vector$ bundle $\mathrm{E}_{\mathbf{G}}$ over $\mathscr{C}(\mathbf{G})$, invariant under complex conjugation. An hermitian vector bundle of rank 1 will be called an hermitian line bundle.

If $f: \mathscr{C}^{\prime} \rightarrow \mathscr{C}$ is a morphism of separated schemes of finite type over $\operatorname{Spec} \mathbf{Z}$, the pull-back by $f$ of an hermitian vector bundle $\overline{\mathrm{E}}:=(\mathrm{E},\|\|$.$) over \mathscr{C}$ is the hermitian vector bundle $f^{*} \overline{\mathrm{E}}$ defined by the locally free sheaf $f^{*} \mathrm{E}$ on $\mathscr{X}^{\prime \prime}$ and the pull-back by $f_{\mathbf{C}}: \mathscr{X}^{\prime}(\mathbf{G}) \rightarrow \mathscr{C}(\mathbf{G})$ of the metric $\|$.$\| , which indeed is a continuous hermitian metric$ on $\left(f^{*} \mathrm{E}\right)_{\mathbf{G}} \simeq f_{\mathbf{G}}^{*} \mathrm{E}_{\mathbf{G}}$. Similarly, various tensorial operations are defined on hermitian vector bundles in an obvious way: from hermitian vector bundles $\overline{\mathrm{E}}, \overline{\mathrm{E}}^{\prime}$ on $\mathscr{X}$; one may form new hermitian vector bundles, such as, for instance, the dual $\check{\mathrm{E}}$, the direct sum $\overline{\mathrm{E}} \oplus \overline{\mathrm{E}}^{\prime}$, the tensor product $\overline{\mathrm{E}} \otimes \overline{\mathrm{E}}^{\prime}$, and the exterior and symmetric powers ${ }^{(11)}$ $\Lambda^{k} \overline{\mathrm{E}}$ and $\mathrm{S}^{k} \overline{\mathrm{E}}$.

When $\mathrm{K}$ is a number field, an hermitian vector bundle over $\operatorname{Spec} \mathscr{O}_{\mathrm{K}}$ is nothing else than a pair $\overline{\mathrm{E}}=\left(\mathrm{E},\left(\|\cdot\|_{\sigma}\right)_{\sigma: \mathrm{K}} \hookrightarrow \mathrm{C}\right)$, where $\mathrm{E}$ is a finitely generated projective $\mathscr{O}_{\mathrm{K}^{-}}$ module $\mathrm{E}$, and $\left(\|\cdot\|_{\sigma}\right)_{\sigma: \mathrm{K}} \hookrightarrow \mathrm{G}$ a family, invariant under complex conjugation, indexed

(11) Whereas there exists a unique sensible definition of hermitian structures on tensor products and on exterior powers of complex vector spaces equipped with hermitian structures, there are several competing conventions currently used in the literature for symmetric powers. We use the following one: if $\mathrm{V}$ is a complex vector space, equipped with an hermitian metric $h$, then, for any integer $d$, the symmetric power $\mathrm{S}^{d} \mathrm{~V}$ is defined as the quotient of the tensor power $\mathrm{V}^{\otimes d}$ and the hermitian metric deduced from $h$ on $\mathrm{S}^{d} \mathrm{~V}$ is defined as the quotient metric of the metric $h^{\otimes d}$ on $\mathrm{V}^{\otimes d}$. 
by the field embeddings of $\mathrm{K}$ in $\mathbf{C}$, of hermitian metrics on the complex vector spaces $\mathrm{E}_{\sigma}:=\mathrm{E} \otimes_{\sigma: O_{\mathrm{K}} \rightarrow \mathbf{C}} \mathbf{C}$.

When $\mathrm{rkE}=1$, the Arakelov degree $\widehat{\operatorname{deg}} \overline{\mathrm{E}}$ of such an hermitian vector bundle over Spec $\mathscr{C}_{\mathrm{K}}$ is defined by the following equality, valid for any element $s$ of $\mathrm{E} \backslash\{0\}$ :

$$
\widehat{\operatorname{deg}} \overline{\mathrm{E}}:=\log \left|\mathrm{E} / \mathscr{C}_{\mathrm{K}} s\right|-\sum_{\sigma: \mathrm{K} \hookrightarrow \mathrm{G}} \log \|s\|_{\sigma}
$$

If, for any $\mathbf{p}$ in $\mathrm{V}_{f}(\mathrm{~K}),\|\|_{\mathbf{p}}$ denotes the $\mathbf{p}$-adic norm on $\mathrm{E}_{\mathrm{K}_{\mathbf{p}}}$ attached to the lattice $\mathrm{E}_{\mathscr{O}_{\mathrm{p}}}$, then we also have, for any $s \in \mathrm{E}_{\mathrm{K}} \backslash\{0\}$ :

$$
\widehat{\operatorname{deg}} \overline{\mathrm{E}}:=-\sum_{\mathbf{p} \in \mathrm{V}_{f}(\mathrm{~K})} \log \|s\|_{\mathbf{p}}-\sum_{\sigma: \mathrm{K} \hookrightarrow \mathbf{C}} \log \|s\|_{\sigma} ;
$$

this does not depend on the choice of $s$ by the product formula.

When rk E is arbitrary, the Arakelov degree is defined as

$$
\widehat{\operatorname{deg}} \overline{\mathrm{E}}:=\widehat{\operatorname{deg}} \Lambda^{\mathrm{rk}} \overline{\mathrm{E}} \text {. }
$$

We shall also use the normalized Arakelov degree of $\overline{\mathrm{E}}$ :

$$
\widehat{\operatorname{deg}}_{n} \overline{\mathrm{E}}:=\frac{1}{[\mathrm{~K}: \mathbf{Q}]} \widehat{\operatorname{deg}} \overline{\mathrm{E}}
$$

When moreover the rank of $\mathrm{E}$ is positive, the slope $\widehat{\mu}(\overline{\mathrm{E}})$ of $\overline{\mathrm{E}}$ is defined as

$$
\widehat{\mu}(\overline{\mathrm{E}}):=\frac{\widehat{\operatorname{deg}}_{n} \overline{\mathrm{E}}}{\mathrm{rkE}}
$$

and its maximal slope $\widehat{\mu}_{\max }(\overline{\mathrm{E}})$ (resp., its minimal slope $\widehat{\mu}_{\text {min }}(\overline{\mathrm{E}})$ ) as the maximum (resp. the minimum) of the slopes $\widehat{\mu}(\overline{\mathrm{F}})$, where $\overline{\mathrm{F}}$ is the hermitian vector bundle defined by a $\mathscr{O}_{\mathrm{K}^{-}}$ submodule (resp. a torsion-free quotient) of positive rank $\mathrm{F}$ of $\mathrm{E}$, equipped with the restrictions to $\mathrm{F}_{\sigma} \subset \mathrm{E}_{\sigma}$ of the hermitian metrics $\left.\left(\|\cdot\|_{\sigma}\right)_{\sigma: K} \hookrightarrow \mathbf{c}\right)$ (resp. with the quotient metrics on the vector spaces $F_{\sigma}$ of these metrics). One easily checks that

$$
\widehat{\mu}_{\max }(\overline{\mathrm{E}})=-\widehat{\mu}_{\min }(\check{\overline{\mathrm{E}}}) \text {. }
$$

The very definition of the Arakelov degree shows that, for any basis $\left(v_{1}, \ldots, v_{\mathrm{rk} E}\right)$ of $\mathrm{E}_{\mathrm{K}}$, whose elements belong to $\mathrm{E}$, we have:

$$
\widehat{\operatorname{deg}} \overline{\mathrm{E}} \geqslant-\sum_{\sigma: \mathrm{K} \hookrightarrow \mathrm{C}} \log \left\|v_{1} \wedge \ldots \wedge v_{\mathrm{rk} E}\right\|_{\sigma} \geqslant-\sum_{\substack{\sigma: \mathrm{K} \\ 1 \leqslant i \leqslant \mathrm{rk} \mathrm{E}}} \log \left\|v_{i}\right\|_{\sigma}
$$

From this inequality, we immediately derive the following lower bound for minimal slopes: 
Lemma 4.1. - For any hermitian vector bundle $\overline{\mathrm{E}}=\left(\mathrm{E},\left(\|\cdot\|_{\sigma}\right)_{\sigma: \mathrm{K}} \longrightarrow \mathrm{C}\right)$ over $\operatorname{Spec} \mathcal{O}_{\mathrm{K}}$ and any finite subset $\mathscr{F}$ of $\mathrm{E}$ which spans the $\mathrm{K}$-vector space $\mathrm{E}_{\mathrm{K}}$, we have:

$$
\widehat{\mu}_{\text {min }}(\overline{\mathbf{E}}) \geqslant-\max _{v \in \mathscr{T}, \sigma: \mathrm{K} \hookrightarrow \mathbf{C}} \log \|v\|_{\sigma} .
$$

From (4.1), it follows that, for any two hermitian line bundles $\overline{\mathrm{L}}_{1}$ and $\overline{\mathrm{L}}_{2}$ on $\operatorname{Spec} \mathscr{C}_{\mathrm{K}}$, we have:

$$
\widehat{\operatorname{deg}} \check{\mathrm{L}}_{1}=-\widehat{\operatorname{deg}} \overline{\mathrm{L}}_{1} \text { and } \widehat{\operatorname{deg}} \overline{\mathrm{L}}_{1} \otimes \overline{\mathrm{L}}_{2}=\widehat{\operatorname{deg}} \overline{\mathrm{L}}_{1}+\widehat{\operatorname{deg}} \overline{\mathrm{L}}_{2} \text {. }
$$

This entails the following properties of the Arakelov degree and slopes:

Lemma 4.2. - (i) Let $\overline{\mathrm{E}}=\left(\mathrm{E},\left(\|\cdot\|_{\overline{\mathrm{E}}, \sigma}\right)_{\sigma: \mathrm{K}} \hookrightarrow \mathrm{C}\right)$ be a hermitian vector bundle over $\operatorname{Spec} \mathcal{O}_{\mathrm{K}}$, and

$$
\{0\}=\mathrm{E}^{\mathrm{N}+1} \subset \mathrm{E}^{\mathrm{N}} \subset \ldots \subset \mathrm{E}^{1} \subset \mathrm{E}^{0}=\mathrm{E}
$$

a filtration of $\mathrm{E}$ by saturated $\mathcal{O}_{\mathrm{K}}$-submodules. If, for any $i \in\{0, \ldots, \mathrm{N}\}, \overline{\mathrm{F}}^{i}$ denotes the subquotient $\mathrm{F}^{i}:=\mathrm{E}^{i} / \mathrm{E}^{i+1}$ equipped with the hermitian metrics quotient of the metrics on $\mathrm{E}_{\sigma}^{i}$ defined by the restrictions of the hermitian metrics $\|\cdot\|_{\overline{\mathrm{E}}, \sigma}$, then we have:

$$
\widehat{\operatorname{deg}} \overline{\mathrm{E}}=\sum_{i=0}^{\mathrm{N}} \widehat{\operatorname{deg}} \overline{\mathrm{F}}^{i}
$$

(ii) For any hermitian vector bundle $\overline{\mathrm{E}}$ and any hermitian line bundle $\overline{\mathrm{L}}$ over Spec $\bigodot_{\mathrm{K}}$, we have:

$$
\widehat{\mu}(\overline{\mathrm{E}} \otimes \overline{\mathrm{L}})=\widehat{\mu}(\overline{\mathrm{E}})+\widehat{\operatorname{deg}}_{n} \overline{\mathrm{L}} \text { and } \widehat{\mu}_{\max }(\overline{\mathrm{E}} \otimes \overline{\mathrm{L}})=\widehat{\mu}_{\max }(\overline{\mathrm{E}})+\widehat{\operatorname{deg}}_{n} \overline{\mathrm{L}} \text {. }
$$

4.1.2. The maximal slope of a symmetric power

In the sequel, we will need some control on the maximal slopes of the successive symmetric powers $\mathrm{S}^{k} \overline{\mathrm{E}}$ of some hermitian vector bundle $\overline{\mathrm{E}}$ over Spec $\mathscr{O}_{\mathrm{K}}$. It is possible to prove that, for any such $\overline{\mathrm{E}}$ and any positive integer $k$, the following upper bound holds:

$$
\widehat{\mu}_{\max }\left(\mathrm{S}^{k} \overline{\mathrm{E}}\right) \leqslant k\left[\widehat{\mu}_{\max }(\overline{\mathrm{E}})+2(\mathrm{rkE}) \log (\mathrm{rk} \mathrm{E})\right] .
$$

(See [Bos98]; see also [Gr01a], Appendix). In this article, the following weak form of (4.6) will be sufficient:

Lemma 4.3. - For any hermitian vector bundle $\overline{\mathrm{E}}$ over $\operatorname{Spec} \mathscr{O}_{\mathrm{K}}$, there exists $c \in \mathbf{R}$ such that, for any positive integer $k$,

$$
\widehat{\mu}_{\max }\left(\mathrm{S}^{k} \overline{\mathrm{E}}\right) \leqslant c k \text {. }
$$


This statement is significantly easier to prove than the more precise estimate (4.6). Indeed, for any positive integer $k$, consider the hermitian vector bundle $\Gamma^{k} \check{\overline{\mathrm{E}}}:=\left(\mathrm{S}^{k} \overline{\mathrm{E}}\right)^{-}$; the underlying $\mathscr{O}_{\mathrm{K}}$-module $\Gamma^{k} \mathrm{E}$ may be identified with the module of invariants under the permutation action of the symmetric group $\mathfrak{S}_{k}$ on the $k$-th tensor power $\check{\mathrm{E}}^{\otimes k}$, and the hermitian metrics on $\Gamma^{k} \check{\overline{\mathrm{E}}}$ coincide with the restrictions of the ones on $\check{\overline{\mathrm{E}}}^{\otimes k}$. Therefore, if $\left(v_{1}, \ldots, v_{e}\right)$ is a basis of $\check{\mathrm{E}}_{\mathrm{K}}$, the elements of which belong to $\check{\mathrm{E}}$, we get a basis

$$
\mathscr{B}_{k}:=\left(v^{i_{1}}, \ldots, i_{\mathrm{N}}\right)_{\substack{\left(i_{1}, \ldots, i_{\mathrm{N}}\right) \in \mathbf{N}^{\mathrm{N}} \\ i_{1}+\ldots+i_{\mathrm{N}}=k}}
$$

of $\Gamma^{k} \check{\mathrm{E}}_{\mathrm{K}}$, formed by elements in $\Gamma^{k} \check{\mathrm{E}}$, by defining $v^{i_{1}, \ldots, i_{\mathrm{N}}}$ as the sum of the elements in the orbit of $v_{1}^{\otimes i_{1}} \otimes \ldots \otimes v_{\mathrm{N}}^{\otimes i_{N}}$ under the symmetric group $\mathfrak{S}_{k}$. The cardinality of this orbit is $k ! / i_{1} ! \ldots i_{\mathrm{N}}$ ! and therefore not larger than $\mathrm{N}^{k}$. This shows that

$$
\max _{w \in \mathscr{B}_{k}, \sigma: \mathrm{K} \hookrightarrow \mathbf{C}}\|w\|_{\sigma} \leqslant \mathrm{N}^{k}\left(\max _{1 \leqslant i \leqslant \mathrm{~N}, \sigma: \mathrm{K} \hookrightarrow \mathbf{C}}\left\|v_{i}\right\|_{\sigma}\right)^{k} .
$$

The upper bound (4.7) now follows from (4.2) and Lemma 4.1.

\subsubsection{Tensor powers of hermitian line bundles and associated hermitian vector bundles of sections}

By a positive Lebesgue measure on a reduced complex analytic space $\mathrm{X}$, of pure dimension $d$, we mean a Radon measure $\mu$ on $\mathrm{X}$ which satisfies the following property: if $i: \mathrm{U} \rightarrow \mathbf{C}^{\mathrm{N}}$ is a holomorphic embedding of an open subset $\mathrm{U}$ of $\mathrm{X}$ and if $\mu_{0}$ denotes the Hausdorff $2 d$-dimensional measure on its image $i(\mathbf{U})$ in $\mathbf{G}^{\mathbf{N}}$ (or equivalently, the measure defined by the current $\omega^{d} \delta_{i(\mathrm{U})}$, where $\left.\omega:=\frac{i}{2} \sum_{k=1}^{\mathrm{N}} d z_{k} \wedge d \bar{z}_{k}\right)$, there exists a Borel function $\lambda: i(\mathbf{U}) \rightarrow \mathbf{R}_{+}^{*}$ such that $i_{*} \mu=\lambda \mu_{0}$ and $\lambda$ and $\lambda^{-1}$ are locally bounded.

Consider now a compact reduced analytic space $\mathrm{X}$, of pure dimension $d$, equipped with a positive Lebesgue measure $\mu$, and $\mathrm{M}$ a holomorphic line bundle over $\mathrm{X}$, endowed with a continuous hermitian metric $\|.\|_{\mathrm{M}}$. For any positive integer $\mathrm{D}$, we may consider the hermitian metric $\|\cdot\|_{\mathrm{M} \otimes \mathrm{D}}$ on $\mathrm{M}^{\otimes \mathrm{D}}$ deduced from $\|.\|_{\mathrm{M}}$ by tensor power, and the associated $\mathrm{L}^{p}$ norms $\|.\|_{\mathrm{D}, \mathrm{L}}{ }^{p}$ on the (finite dimensional) complex vector space $\Gamma\left(\mathrm{X}, \mathbf{M}^{\otimes \mathrm{D}}\right)$ : for any $s \in \Gamma\left(\mathrm{X}, \mathbf{M}^{\otimes D}\right)$, we let

$$
\|s\|_{\mathrm{D}, \mathrm{L}^{\infty}}:=\max _{x \in \mathrm{X}}\|s(x)\|_{\mathrm{M} \otimes \mathrm{D}}
$$

and, for any $p \in[1,+\infty[$,

$$
\|s\|_{\mathrm{D}, \mathrm{L}}{ }^{p}:=\left(\int_{\mathrm{X}}\|s(x)\|_{\mathrm{M}^{\otimes \mathrm{D}}}^{p} d \mu(x)\right)^{1 / p} .
$$


These norms are easily compared. On one hand, we have, as a special case of general estimates relating $\mathrm{L}^{p}$-norms of measurable functions on a measured space, for any integer $\mathrm{D}$, any $s \in \Gamma\left(\mathrm{X}, \mathrm{M}^{\otimes \mathrm{D}}\right)$, and any $p \in[1,+\infty[$ :

$$
\mu(\mathrm{X})^{-1}\|s\|_{\mathrm{D}, \mathrm{L}^{1}} \leqslant \mu(\mathrm{X})^{-1 / p}\|s\|_{\mathrm{D}, \mathrm{L}^{p}} \leqslant\|s\|_{\mathrm{D}, \mathrm{L}^{\infty}}
$$

On the other hand, from the elementary local properties of analytic spaces and holomorphic functions and the compactness of $\mathrm{X}$, one easily derives the existence of some constant $\mathrm{C} \in[1,+\infty$ [ such that, for any positive integer $\mathrm{D}$ and any $s \in \Gamma\left(\mathrm{X}, \mathrm{M}^{\otimes \mathrm{D}}\right)$, the following estimate holds:

$$
\|s\|_{\mathrm{D}, \mathrm{L}^{\infty}} \leqslant \mathrm{C}^{\mathrm{D}}\|s\|_{\mathrm{D}, \mathrm{L}^{1}} .
$$

Let now $\mathscr{C}$ be an integral separated proper flat scheme of finite type over Spec Z, endowed with a hermitian line bundle $\overline{\mathscr{D}}$ and with a positive Lebesgue measure $\mathrm{v}$, invariant under complex conjugation, over $\mathscr{C}(\mathbf{G})=\coprod_{\sigma: \mathrm{K}} \hookrightarrow \mathrm{G} \mathscr{\mathscr { C }}{ }_{\sigma}(\mathbf{G})$. For any positive integer $\mathrm{D}$, let $\overline{\mathrm{E}}_{\mathrm{D}}$ be the hermitian vector bundle over Spec $\mathscr{O}_{\mathrm{K}}$ defined by the $\mathscr{O}_{\mathrm{K}}$-module $\mathrm{E}_{\mathrm{D}}:=\Gamma\left(\mathscr{C}, \mathscr{B}{ }^{\otimes \mathrm{D}}\right)$ equipped with the hermitian structure $\left(\|\cdot\|_{\sigma}\right)_{\sigma: \mathrm{K}} \hookrightarrow \mathrm{G}$, where $\|.\|_{\sigma}$ denotes the $\mathrm{L}^{2}$-norm on $\mathrm{E}_{\mathrm{D}, \sigma} \simeq \Gamma\left(\mathscr{C}_{\sigma}, \mathscr{C}_{\sigma}^{\otimes \mathrm{D}}\right)$ defined by the construction above, applied to $\mathrm{X}=\mathscr{C}_{\sigma}, \mathrm{M}=\mathscr{C}_{\sigma}, \mu=\mathrm{v}_{\mid \mathscr{X}_{\sigma}}$ and $p=2$. Various authors have studied in depth and given important consequences of the behaviour when $\mathrm{D}$ goes to infinity of the Arakelov degree (or equivalently, of the Arakelov slope) of such hermitian vector bundles of sections of powers of an hermitian line bundle $\overline{\mathscr{B}}$, provided additional conditions are satisfied, such as suitable positivity properties of $\overline{\mathscr{B}}$, or the smoothness of $\mathscr{C}_{\mathrm{K}}$ (see for instance [GS92] and [Zha98] for discussions and references). In this article, we shall be content with a crude, but very general, estimate:

Proposition 4.4. - With the notation above, if the line bundle $\mathscr{D}_{\mathrm{K}}$ on $\mathscr{C}_{\mathrm{K}}$ is ample, then there exists $c \in \mathbf{R}_{+}^{*}$ such that, for any positive integer $\mathrm{D}$,

$$
\widehat{\mu}\left(\overline{\mathrm{E}}_{\mathrm{D}}\right) \geqslant-c \mathrm{D} \text {. }
$$

Proof. - Since $\mathscr{B}_{\mathrm{K}}$ is ample, the graded K-algebra

$$
\bigoplus_{\mathrm{D} \in \mathbf{N}} \mathrm{E}_{\mathrm{D}, \mathrm{K}}=\bigoplus_{\mathrm{D} \in \mathbf{N}} \Gamma\left(\mathscr{C}_{\mathrm{K}}, \mathscr{B}_{\mathrm{K}}^{\otimes \mathrm{D}}\right)
$$

is finitely generated. Therefore, we can find a finite family $\left(s_{i}\right)_{i \in \mathrm{I}}$, where $s_{i}$ is an element of $\Gamma\left(\mathscr{B}, \mathscr{D}^{\otimes \mathrm{D}_{i}}\right)$ for some positive integer $\mathrm{D}_{i}$, such that, for any positive integer $\mathrm{D}$, the monomials of weight $\mathrm{D}$ in the $s_{i}$ 's $\left(s_{i}\right.$ being attributed the weight $\mathrm{D}_{i}$ ) span $\mathrm{E}_{\mathrm{D}, \mathrm{K}}$. The estimates (4.8) and (4.9) relating $\mathrm{L}^{2}$ and $\mathrm{L}^{\infty}$ norms on the complex vectors spaces $\mathrm{E}_{\mathrm{D}, \sigma}$ show that, if $\mathrm{C} \in[1,+\infty$ [ is large enough, then, for any such monomial $s$ and any embedding $\sigma: \mathrm{K} \hookrightarrow \mathbf{C}$, we have $\|s\|_{\sigma} \leqslant \mathrm{C}^{\mathrm{D}}$. Then Lemma 4.1 shows that $\widehat{\mu}\left(\bar{E}_{\mathrm{D}}\right) \geqslant-(\log \mathrm{C}) \mathrm{D}$. 
Observe that the preceding proof actually establishes a stronger and more general result: if the graded algebra $\bigoplus_{\mathrm{D} \in \mathbf{N}} \Gamma\left(\mathscr{C}_{\mathrm{K}}, \mathscr{D}_{\mathrm{K}}^{\otimes \mathrm{D}}\right)$ is finitely generated, then there exists $c \in \mathbf{R}_{+}^{*}$ such that, for any positive integer $\mathrm{D}, \widehat{\mu}_{\text {min }}\left(\overline{\mathrm{E}}_{\mathrm{D}}\right) \geqslant-c \mathrm{D}$.

\subsubsection{Heights of linear maps and slope inequalities}

A simple slope inequality. - Let $\overline{\mathrm{E}}=\left(\mathrm{E},\left(\|\cdot\|_{\overline{\mathrm{E}}, \sigma}\right)_{\sigma: \mathrm{K}} \hookrightarrow \mathbf{G}\right)$ and $\overline{\mathrm{F}}=\left(\mathrm{F},\left(\|\cdot\|_{\overline{\mathrm{F}}, \sigma}\right)_{\sigma: \mathrm{K}} \hookrightarrow \mathbf{G}\right)$ be two hermitian vector bundles over $\operatorname{Spec} \mathscr{C}_{\mathrm{K}}$. For any $\mathbf{p}$ in $\mathrm{V}_{f}(\mathrm{~K})$, let \|\|$_{\mathbf{p}}$ be the p-adic norm on $\operatorname{Hom}_{\mathrm{K}_{\mathrm{p}}}\left(\mathrm{E}_{\mathrm{K}_{\mathrm{p}}}, \mathrm{F}_{\mathrm{K}_{\mathrm{p}}}\right) \simeq \check{\mathrm{E}}_{\mathrm{K}_{\mathrm{p}}} \otimes \mathrm{F}_{\mathrm{K}_{\mathrm{p}}}$ attached to its $\mathscr{Q}_{\mathrm{p}}$-lattice $\check{\mathrm{E}}_{\mathscr{O}_{\mathrm{p}}} \otimes \mathrm{F}_{\mathscr{O}_{\mathrm{p}}}$, and for any embedding $\sigma: \mathrm{K} \hookrightarrow \mathbf{C}$, let $\|\cdot\|_{\sigma}$ be the operator norm on $\operatorname{Hom}_{\mathbf{C}}\left(\mathrm{E}_{\sigma}, \mathrm{F}_{\sigma}\right)$ defined from the hermitian norms $\|\cdot\|_{\overline{\mathrm{E}}, \sigma}$ and $\|\cdot\|_{\overline{\mathrm{F}}, \sigma}{ }^{(12)}$.

For any non-zero K-linear map $\varphi: \mathrm{E}_{\mathrm{K}} \mapsto \mathrm{F}_{\mathrm{K}}$, we define the height of $\varphi$ with respect to $\overline{\mathrm{E}}$ and $\overline{\mathrm{F}}$ as the real number:

$$
h(\overline{\mathrm{E}}, \overline{\mathrm{F}}, \varphi):=\frac{1}{[K: \mathbf{Q}]}\left[\sum_{\mathbf{p} \in \mathrm{V}_{f}(\mathrm{~K})} \log \|\varphi\|_{\mathbf{p}}+\sum_{\sigma: \mathrm{K} \hookrightarrow \mathrm{C}} \log \|\varphi\|_{\sigma}\right] .
$$

In the proof below, the following inequalities will play the role of Siegel's Lemma.

Proposition 4.5. - With the notation above, if moreover $\varphi$ is injective, then

$$
\widehat{\operatorname{deg}}_{n} \overline{\mathrm{E}} \leqslant \operatorname{rkE}\left[\widehat{\mu}_{\max }(\overline{\mathrm{F}})+h(\overline{\mathrm{E}}, \overline{\mathrm{F}}, \varphi)\right]
$$

Proof. - Let $\mathrm{F}^{\prime}$ be the saturated $\mathscr{O}_{\mathrm{K}}$-submodule of $\mathrm{F}$ such that $\mathrm{F}_{\mathrm{K}}^{\prime}=\varphi\left(\mathrm{E}_{\mathrm{K}}\right)$. Equipped with the restrictions of the hermitian metrics $\|\cdot\|_{\overline{\mathrm{F}}, \sigma}$, it defines an hermitian vector bundle $\overline{\mathrm{F}}^{\prime}$ on $\operatorname{Spec} \mathscr{C}_{\mathrm{K}}$, of positive $\operatorname{rank} r:=\operatorname{rkE}$. The map $\varphi: \mathrm{E}_{\mathrm{K}} \rightarrow \mathrm{F}_{\mathrm{K}}^{\prime}$ is bijective, and therefore $\Lambda^{r} \varphi: \Lambda^{r} \mathrm{E}_{\mathrm{K}} \rightarrow \Lambda^{r} \mathrm{~F}_{\mathrm{K}}^{\prime}$ defines a non-zero element of $\mathrm{L}_{\mathrm{K}}$, the one dimensional K-vector space underlying the hermitian line bundle $\overline{\mathrm{L}}:=\Lambda^{r} \check{\overline{\mathrm{E}}} \otimes \Lambda^{r} \overline{\mathrm{F}}$. Therefore, according to (4.3) and (4.1), we have:

$$
\begin{aligned}
\widehat{\operatorname{deg}} \overline{\mathrm{F}}^{\prime}-\widehat{\operatorname{deg}} \overline{\mathrm{E}} & =\widehat{\operatorname{deg}} \overline{\mathrm{L}} \\
& =-\sum_{\mathbf{p} \in \mathrm{V}_{f}(\mathrm{~K})} \log \left\|\Lambda^{r} \varphi\right\|_{\mathbf{p}}-\sum_{\sigma: \mathrm{K} \hookrightarrow \mathbf{G}} \log \left\|\Lambda^{r} \varphi\right\|_{\sigma},
\end{aligned}
$$

where \|\|$_{\mathbf{p}}$ and \|\|$_{\sigma}$ denote the $\mathbf{p}$-adic and hermitian norms on $\mathrm{L}_{\mathrm{K}_{\mathbf{p}}}$ and $\mathrm{L}_{\sigma}$ attached

\footnotetext{
(12) In other words, for any $\mathrm{T} \in \operatorname{Hom}_{\mathbf{C}}\left(\mathrm{E}_{\boldsymbol{\sigma}}, \mathrm{F}_{\boldsymbol{\sigma}}\right),\|\mathrm{T}\|_{\boldsymbol{\sigma}}=\max _{x \in \mathrm{E}_{\boldsymbol{\sigma}} \backslash\{0\}} \frac{\|\mathrm{T} x\|_{\overline{\mathrm{F}}, \boldsymbol{\sigma}}}{\|x\|_{\overline{\mathrm{E}}, \boldsymbol{\sigma}}}$. Observe that the norms \|\|$_{\mathbf{p}}$ could be similarly defined as the operator norms on $\operatorname{Hom}_{\mathrm{K}_{\mathbf{p}}}\left(\mathrm{E}_{\mathrm{K}_{\mathbf{p}}}, \mathrm{F}_{\mathrm{K}_{\mathbf{p}}}\right)$ defined from the $\mathbf{p}$-adic norms on $\mathrm{E}_{\mathrm{K}_{\mathbf{p}}}$ and $\mathrm{F}_{\mathrm{K}_{\mathbf{p}}}$ attached to the lattices $\mathrm{E}_{\mathscr{C}_{\mathbf{p}}}$ and $\mathrm{F}_{\mathscr{C}_{\mathbf{p}}}$.
} 
to the hermitian line bundle $\overline{\mathrm{L}}$. Together with the inequalities $\left\|\Lambda^{r} \varphi\right\|_{\mathbf{p}} \leqslant\|\varphi\|_{\mathbf{p}}^{r}$ and $\left\|\Lambda^{r} \varphi\right\|_{\sigma} \leqslant\|\varphi\|_{\sigma}^{r}$, this shows that

$$
\widehat{\operatorname{deg}}_{n} \overline{\mathrm{E}}-\widehat{\operatorname{deg}}_{n} \overline{\mathrm{F}}^{\prime} \leqslant r h(\overline{\mathrm{E}}, \overline{\mathrm{F}}, \varphi) .
$$

Besides, the definition of $\widehat{\mu}_{\max }(\overline{\mathbf{F}})$ shows that

$$
\widehat{\operatorname{deg}}_{n} \overline{\mathrm{F}}^{\prime} \leqslant r \widehat{\mu}_{\max }(\overline{\mathbf{F}}) .
$$

The inequality (4.11) follows from (4.12) and (4.13).

It is convenient to define the maximal slope $\widehat{\mu}_{\max }(\overline{\mathbf{F}})$ of an hermitian vector bundle $\overline{\mathrm{F}}$ of rank 0 and the height $h(\overline{\mathrm{E}}, \overline{\mathrm{F}}, \varphi)$ of the linear map $\varphi=0$ to be $-\infty$. Then (4.11) holds for any injective linear map $\varphi$, even if $\varphi$ vanishes, provided we follow the usual convention $0 .(-\infty)=0$ to define its right-hand side in that case.

Filtered vector bundles. - We now state a variant of the slope inequality (4.11) for injective maps which involves filtered vector bundles over $\mathrm{S}:=\operatorname{Spec} \mathscr{O}_{\mathrm{K}}$ and is particularly convenient for applications.

Let $\mathrm{F}_{\mathrm{K}}$ be a finite dimensional $\mathrm{K}$-vector space equipped with a filtration

$$
\{0\}=\mathrm{F}_{\mathrm{K}}^{\mathrm{N}+1} \subset \mathrm{F}_{\mathrm{K}}^{\mathrm{N}} \subset \ldots \subset \mathrm{F}_{\mathrm{K}}^{1} \subset \mathrm{F}_{\mathrm{K}}^{0}=\mathrm{F}_{\mathrm{K}}
$$

by $\mathrm{K}$-vector subspaces. Assume moreover that, for every $i \in\{0, \ldots, \mathrm{N}\}$, the subquotients $\mathrm{G}_{\mathrm{K}}^{i}:=\mathrm{F}_{\mathrm{K}}^{i} / \mathrm{F}_{\mathrm{K}}^{i+1}$ are the underlying $\mathrm{K}$-vector spaces of some hermitian vector bundles $\overline{\mathrm{G}}^{i}:=\left(\mathrm{G}^{i},\left(\|\cdot\|_{\sigma}\right)_{\sigma: \mathrm{K}} \hookrightarrow \mathrm{G}\right)$ over $\mathrm{S}:=\operatorname{Spec} \mathcal{O}_{\mathrm{K}}$. If $\overline{\mathrm{E}}=\left(\mathrm{E},\left(\|\cdot\|_{\overline{\mathrm{E}}, \sigma}\right)_{\sigma: \mathrm{K}} \hookrightarrow \mathbf{C}\right)$ is another hermitian vector bundle over $\mathrm{S}$ and $\varphi: \mathrm{E}_{\mathrm{K}} \rightarrow \mathrm{F}_{\mathrm{K}}$ an injective K-linear map, then, for any $i \in\{0, \ldots, \mathrm{N}+1\}$, we may define $\mathrm{E}_{\mathrm{K}}^{i}:=\varphi^{-1}\left(\mathrm{~F}_{\mathrm{K}}^{i}\right)$ and $\mathrm{E}^{i}:=\mathrm{E}_{\mathrm{K}}^{i} \cap \mathrm{E}$. Then the $\mathscr{O}_{\mathrm{K}}$-submodules $\mathrm{E}^{i}$ define a filtration

$$
\{0\}=\mathrm{E}^{\mathrm{N}+1} \subset \mathrm{E}^{\mathrm{N}} \subset \ldots \subset \mathrm{E}^{1} \subset \mathrm{E}^{0}=\mathrm{E}
$$

and, equipped with the restrictions of the hermitian structures on $\overline{\mathrm{E}}$, become hermitian vector bundles over S. Finally, for any $i \in\{0, \ldots, \mathrm{N}\}$, let us consider the linear map $\varphi^{i}: \mathrm{E}_{\mathrm{K}}^{i} \rightarrow \mathrm{G}_{\mathrm{K}}^{i}$ defined by composing the restriction $\varphi_{\mid \mathrm{E}_{\mathrm{K}}^{i}}: \mathrm{E}_{\mathrm{K}}^{i} \rightarrow \mathrm{F}_{\mathrm{K}}^{i}$ and the quotient $\operatorname{map} p_{i}: \mathrm{F}_{\mathrm{K}}^{i} \rightarrow \mathrm{G}_{\mathrm{K}}^{i}$.

Proposition 4.6. - With the above notation, we have:

$$
\widehat{\operatorname{deg}}_{n} \overline{\mathrm{E}} \leqslant \sum_{i=0}^{\mathrm{N}} \operatorname{rk}\left(\mathrm{E}^{i} / \mathrm{E}^{i+1}\right)\left[\widehat{\mu}_{\max }\left(\overline{\mathrm{G}}^{i}\right)+h\left(\overline{\mathrm{E}}^{i}, \overline{\mathrm{G}}^{i}, \varphi^{i}\right)\right] .
$$

Proof. - For any $i \in\{0, \ldots, \mathrm{N}\}$, let $\overline{\mathrm{E}^{i} / \mathrm{E}^{i+1}}$ be the subquotient $\mathrm{E}^{i} / \mathrm{E}^{i+1}$ equipped with the hermitian metrics quotient of the metrics on $\mathrm{E}_{\sigma}^{i}$ defined by the restrictions of 
the hermitian metrics $\|\cdot\|_{\overline{\mathrm{E}}, \sigma}$. Then, according to (4.4), we have:

$$
\widehat{\operatorname{deg}}_{n} \overline{\mathrm{E}}=\sum_{i=0}^{\mathrm{N}} \widehat{\operatorname{deg}}_{n} \overline{\mathrm{E}^{i} / \mathrm{E}^{i+1}} .
$$

Moreover, for any $i \in\{0, \ldots, \mathrm{N}\}$, the map $\varphi^{i}$ vanishes on $\mathrm{E}_{\mathrm{K}}^{i+1}$ and may be written as the the composition of the quotient map $\mathrm{E}_{\mathrm{K}}^{i} \rightarrow \mathrm{E}_{\mathrm{K}}^{i} / \mathrm{E}_{\mathrm{K}}^{i+1}$ and of some (unique) map $\widetilde{\varphi}^{i}: \mathrm{E}_{\mathrm{K}}^{i} / \mathrm{E}_{\mathrm{K}}^{i+1} \rightarrow \mathrm{G}_{\mathrm{K}}^{i}$. The very definition of the filtration $\{0\}=\mathrm{E}_{\mathrm{K}}^{\mathrm{N}+1} \subset \mathrm{E}_{\mathrm{K}}^{\mathrm{N}} \subset \ldots \subset \mathrm{E}_{\mathrm{K}}^{1} \subset \mathrm{E}_{\mathrm{K}}^{0}=\mathrm{E}_{\mathrm{K}}$ shows that the maps $\widetilde{\varphi}^{i}$ are injective. Therefore, by Proposition 4.5,

$$
\widehat{\operatorname{deg}}_{n} \overline{\mathrm{E}^{i} / \mathrm{E}^{i+1}} \leqslant \operatorname{rk}\left(\mathrm{E}_{\mathrm{K}}^{i} / \mathrm{E}_{\mathrm{K}}^{i+1}\right)\left[\widehat{\mu}_{\max }\left(\overline{\mathrm{G}}^{i}\right)+h\left(\overline{\mathrm{E}^{i} / \mathrm{E}^{i+1}}, \overline{\mathrm{G}}^{i}, \widetilde{\varphi}^{i}\right)\right] .
$$

Finally, for any $i \in\{0, \ldots, \mathrm{N}\}$, we have

$$
h\left(\overline{\mathrm{E}^{i} / \mathrm{E}^{i+1}}, \overline{\mathrm{G}}^{i}, \widetilde{\varphi}^{i}\right)=h\left(\overline{\mathrm{E}}^{i}, \overline{\mathrm{G}}^{i}, \varphi^{i}\right) .
$$

Indeed, the local norms of $\widetilde{\varphi}^{i}$ and $\varphi^{i}$ coincide.

Inequality (4.6) follows from (4.15), (4.16), and (4.17).

4.2. Diophantine approximation using slopes and heights of evaluation maps

We now return to the notation of Theorem 3.4, which will be proved in this subsection and the following one.

\subsubsection{Notation and preliminary reductions}

We already observed that, if $\mathrm{U}$ is any open subvariety of $\mathrm{X}$ containing $\mathrm{P}$, the statement of Theorem 3.4 is equivalent to the same statement where $\mathrm{X}$ has been replaced by $\mathrm{U}$. This shows that we may assume that $\mathrm{X}$ is projective over $\mathrm{K}$.

For any $n \in \mathbf{N}$, let $\mathrm{V}_{n}$ be the $n$-th infinitesimal neighbourhood of $\mathrm{P}$ in $\hat{\mathrm{V}}$. Thus we have:

$$
\mathrm{V}_{0}=\{\mathrm{P}\} \subset \mathrm{V}_{1} \subset \ldots \subset \mathrm{V}_{n} \subset \mathrm{V}_{n+1} \subset \ldots
$$

and

$$
\hat{\mathrm{V}}=\lim _{\vec{n}} \mathrm{~V}_{n}
$$

It will also be convenient to let $\mathrm{V}_{-1}:=\emptyset$.

Clearly, we may assume that the the dimension $v$ of $\hat{\mathrm{V}}$ is positive. 
Let us introduce the Zariski closure $\mathrm{Y}$ of $\hat{\mathrm{V}}$ in $\mathrm{X}$, namely the smallest closed subscheme $\mathrm{Y}$ of $\mathrm{X}$ containing all the $\mathrm{V}_{n}$, or, equivalently, its smallest closed subscheme containing $\mathrm{P}$ such that $\hat{\mathrm{Y}}_{\mathrm{P}}$ contains $\hat{\mathrm{V}}$. It is an integral subscheme of $\mathrm{X}$, and the conclusion of Theorem 3.4 precisely asserts that $\hat{\mathrm{V}}$ is a branch of this subscheme at $\mathrm{P}$. By replacing $\mathrm{X}$ by $\mathrm{Y}$, we may assume that $\hat{\mathrm{V}}$ is Zariski dense in $\mathrm{X}$, and then we have to show that, under the above hypotheses, the dimension $v$ of $\hat{\mathrm{V}}$ coincides with the dimension $d$ of $\mathrm{X}$.

To achieve this, let us choose a projective model $\mathscr{C}$ for $\mathrm{X}$ over $\operatorname{Spec} \mathscr{C}_{\mathrm{K}}$ (i.e., a projective integral scheme over $\operatorname{Spec} \mathscr{O}_{\mathrm{K}}$ whose generic fiber $\mathscr{C}{ }_{\mathrm{K}}$ coincides with $\mathrm{X}$ ), and an hermitian line bundle $\overline{\mathscr{C}}$ over $\mathscr{C}$, whose restriction $\mathrm{L}:=\mathscr{B}_{\mathrm{K}}$ to $\mathrm{X}$ is ample. Let us also choose a positive Lebesgue measure $\mu$ on $\mathrm{X}(\mathbf{G})$, invariant under complex conjugation, and a family $\left(\|\cdot\|_{\sigma}\right)_{\sigma: \mathrm{K}} \hookrightarrow \mathrm{C}$, invariant by complex conjugation, of hermitian metrics on the complex vector spaces $\left(\mathrm{T}_{\mathrm{P}} \hat{\mathrm{V}}_{\sigma}\right)_{\sigma: \mathrm{K}} \hookrightarrow \mathrm{G}$.

For any $\mathrm{D} \in \mathbf{N}$, we let

$$
\mathrm{E}_{\mathrm{D}}:=\Gamma\left(\mathscr{X} ; \mathscr{D}^{\otimes \mathrm{D}}\right) \text {. }
$$

It is a finitely generated projective $\mathscr{C}_{\mathrm{K}}$-module. For any field embedding $\sigma: \mathrm{K} \hookrightarrow \mathbf{C}$ and any integer $\mathrm{D}$, the holomorphic line bundle $\mathrm{L}_{\sigma}^{\otimes \mathrm{D}}$ on $\mathrm{X}_{\sigma}(\mathbf{C})$ is endowed with the D-th tensorial power of the hermitian metric defining $\overline{\mathscr{C}}$. We shall denote by \|\|$_{\mathrm{L}^{\infty}, \sigma}$ (resp. by \|\|$\left._{\mathrm{L}^{2}, \sigma}\right)$ the associated $\mathrm{L}^{\infty}$-norm on $\mathrm{E}_{\mathrm{D}, \sigma} \simeq \Gamma\left(\mathrm{X}_{\sigma}, \mathrm{L}_{\sigma}^{\otimes \mathrm{D}}\right.$ ) (resp. the associated $\mathrm{L}^{2}$-norm on $\mathrm{E}_{\mathrm{D}, \sigma}$ defined by means of the Lebesgue measure $\mu_{\mid \mathrm{X}_{\sigma}}$ ).

Since the structural morphism $\pi: \mathscr{C} \rightarrow$ Spec $\mathscr{O}_{\mathrm{K}}$ is proper, the rational point $\mathrm{P} \in \mathrm{X}(\mathrm{K})=\mathscr{C}(\mathrm{K})$ extends to a section $\mathscr{P}$ of $\pi$. Let $\check{t}$ be the image of the $\mathscr{O}_{\mathrm{K}}$-module $\mathscr{P}^{*} \Omega_{\mathscr{X} / \mathscr{C}_{\mathrm{K}}}^{1}$ by the composite map

$$
\mathscr{P}^{*} \Omega_{\mathscr{C} / \mathscr{O}_{\mathrm{K}}}^{1} \rightarrow\left(\mathscr{P}^{*} \Omega_{\mathscr{X} / \mathscr{O}_{\mathrm{K}}}^{1}\right)_{\mathrm{K}} \simeq \Omega_{\mathrm{X} / \mathrm{K}, \mathrm{P}}^{1} \rightarrow \check{\mathrm{T}}_{\mathrm{P}} \hat{\mathrm{V}}
$$

It is a finitely generated projective $\mathscr{C}_{\mathrm{K}}$-module, and $\check{t}_{\mathrm{K}}$ may be identified with $\check{\mathrm{T}}_{\mathrm{P}} \hat{\mathrm{V}}$. Equipped with the dual metrics of the metrics \|\|$_{\sigma}$ on the complex vector spaces $\mathrm{T}_{\mathrm{P}} \hat{\mathrm{V}}_{\sigma}$, it defines an hermitian vector bundle $\check{\bar{t}}$ on $\operatorname{Spec} \mathscr{C}_{\mathrm{K}}$.

As hinted in the introduction, the proof of Theorem 3.4 is based on the consideration of the restriction maps

$$
\varphi_{\mathrm{D}, n}: \mathrm{E}_{\mathrm{D}, \mathrm{K}}:=\Gamma\left(\mathrm{X}, \mathrm{L}^{\otimes \mathrm{D}}\right) \longrightarrow \mathrm{L}_{\mid \mathrm{V}_{n}}^{\otimes \mathrm{D}},
$$

for "large values" of $(\mathrm{D}, n) \in \mathbf{N} \times \mathbf{N}$. 


\subsubsection{Slope inequalities}

Observe that, for any $\mathrm{D}$ and for $n$ large enough (say, $\left.n \geqslant n_{0}(\mathrm{D})\right), \varphi_{\mathrm{D}, n}$ is injective. This directly follows from (indeed, is equivalent to) the Zariski density of $\hat{V}$ in $X$. Moreover, $\mathscr{C}_{\mathrm{V}_{n}}$ has a natural filtration, by the order of vanishing at $\mathrm{P}$. The successive subquotients $\operatorname{ker}\left(\mathrm{L}_{\mid \mathrm{V}_{i}}^{\otimes \mathrm{D}} \rightarrow \mathrm{L}_{\mid \mathrm{V}_{i-1}}^{\otimes \mathrm{D}}\right)$ associated to this filtration may be identified with the tensor products $\mathrm{S}^{i} \check{t}_{\mathrm{K}} \otimes \mathrm{L}_{\mathrm{P}}^{\otimes \mathrm{D}}$. The inverse image of this filtration by $\varphi_{\mathrm{D}, n}$ is the filtration

$$
\mathrm{E}_{\mathrm{D}}^{i}:=\left\{s \in \mathrm{E}_{\mathrm{D}} \mid s_{\mid \mathrm{V}_{i-1}}=0\right\}
$$

of $\mathrm{E}_{\mathrm{D}}$. Moreover, for any $i \in\{0, \ldots, n\}$, the map

$$
\varphi_{\mathrm{D}}^{i}: \mathrm{E}_{\mathrm{D}, \mathrm{K}}^{i} \longrightarrow \operatorname{ker}\left(\mathrm{L}_{\mid \mathrm{V}_{i}}^{\otimes \mathrm{D}} \rightarrow \mathrm{L}_{\mid \mathrm{V}_{i-1}}^{\otimes \mathrm{D}}\right) \simeq \mathrm{S}^{i} \check{t}_{\mathrm{K}} \otimes \mathrm{L}_{\mathrm{P}}^{\otimes \mathrm{D}}
$$

deduced from $\varphi_{\mathrm{D}, n}$ is the map which maps a section of $\mathrm{L}^{\otimes \mathrm{D}}$ on $\mathrm{X}$ which vanishes on $\mathrm{V}_{i-1}$ to its jet of order $i$ along $\hat{\mathrm{V}}$, identified with an element of $\mathrm{S}^{i} \check{t}_{\mathrm{K}} \otimes \mathrm{L}_{\mathrm{P}}^{\otimes \mathrm{D}}$.

We may apply the slope inequality for filtered vector bundles in Proposition 4.6 to $\varphi_{\mathrm{D}, n}: \mathrm{E}_{\mathrm{D}, \mathrm{K}} \rightarrow \mathcal{Q}_{\mathrm{V}_{n}}$, for $n \geqslant n_{0}(\mathrm{D})$, the $\mathrm{K}$-vector space $\mathcal{Q}_{\mathrm{V}_{n}}$ being filtered by the order of vanishing. Since the associated subquotients may be identified to $\mathrm{S}^{i} \check{t}_{\mathrm{K}} \otimes \mathrm{L}_{\mathrm{P}}^{\otimes \mathrm{D}}$, which is the underlying $\mathrm{K}$-vector space of the hermitian vector bundle $\mathrm{S}^{i} \check{\bar{t}} \otimes \mathscr{P}^{*} \overline{\mathscr{C}}^{\otimes \mathrm{D}}$, we get:

$$
\widehat{\operatorname{deg}}_{n} \overline{\mathrm{E}}_{\mathrm{D}} \leqslant \sum_{i \geqslant 0} \mathrm{rk}\left(\mathrm{E}_{\mathrm{D}}^{i} / \mathrm{E}_{\mathrm{D}}^{i+1}\right)\left[\widehat{\mu}_{\max }\left(\mathrm{S}^{i \check{\bar{t}}} \otimes \mathscr{P}^{*} \overline{\mathscr{D}}^{\otimes \mathrm{D}}\right)+h\left(\overline{\mathrm{E}}_{\mathrm{D}}^{i}, \mathrm{~S}^{i \check{t}} \otimes \mathscr{P}^{*} \overline{\mathscr{C}}^{\otimes \mathrm{D}}, \varphi_{\mathrm{D}}^{i}\right)\right] .
$$

Since $\mathrm{E}_{\mathrm{D}}^{i}=\{0\}$ for $i>n_{0}(\mathrm{D})$, the sum is indeed finite and coincides with the sum over $i$ in the finite interval $\{0, \ldots, n\}$, for any $n \geqslant n_{0}(\mathrm{D})$.

The proof of Theorem 3.4 will easily follow from the inequality (4.18), together with the estimates on the quantities which occur in it given in the next two lemma.

Lemma 4.7. - 1) For any $(\mathrm{D}, \imath) \in \mathbf{N} \times \mathbf{N}$,

$$
\mathrm{rkE}_{\mathrm{D}} / \mathrm{E}_{\mathrm{D}}^{i} \leqslant \mathrm{rkL}_{\mid \mathrm{V}_{i-1}}=\mathrm{rk} \mathcal{O}_{\mid \mathrm{V}_{i-1}}=\frac{(i+v-1) \ldots(i+1) i}{v !} \leqslant i .
$$

2) If $\operatorname{deg} \mathrm{X}$ denotes the degree of $\mathrm{X}$ with respect to $\mathrm{L}$, then, when $\mathrm{D}$ goes to infinity,

$$
\mathrm{rkE}_{\mathrm{D}} \sim \frac{1}{d !} \operatorname{deg} \mathrm{XD}^{d}
$$

These geometric estimates are elementary: assertion 1) follows from the very definition of $\mathrm{E}_{\mathrm{D}, \mathrm{K}}^{i}$ as the kernel of $\varphi_{\mathrm{D}, i-1}: \mathrm{E}_{\mathrm{D}, \mathrm{K}} \rightarrow \mathrm{L}_{\mid \mathrm{V}_{i-1}}$; assertion 2) is basically the definition of $\operatorname{deg} \mathrm{X}$ by means of the Hilbert-Samuel polynomial. 
Lemma 4.8. - 1) There exists $c_{1}>0$ such that, for any $\mathrm{D} \in \mathbf{N} \backslash\{0\}$,

$$
\widehat{\mu}\left(\overline{\mathrm{E}}_{\mathrm{D}}\right) \geqslant-c_{1} \mathrm{D} \text {. }
$$

2) There exists $c_{2}>0$ such that, for any $(\mathrm{d}, i) \in(\mathbf{N} \backslash\{0\}) \times \mathbf{N}$,

$$
\widehat{\mu}_{\max }\left(\mathrm{S}^{i \check{\bar{t}}} \otimes \mathscr{P}^{*} \overline{\mathscr{D}}^{\otimes \mathrm{D}}\right) \leqslant c_{2}(i+\mathrm{D}) .
$$

3) There exists a function $\tau: \mathbf{R}_{+} \rightarrow \mathbf{R}$, bounded from below, which satisfies

$$
\lim _{x \rightarrow+\infty} \frac{\tau(x)}{x}=+\infty
$$

and, for any $(\mathbf{D}, \imath) \in(\mathbf{N} \backslash\{0\}) \times \mathbf{N}$,

$$
h\left(\overline{\mathrm{E}}_{\mathrm{D}}^{i}, \mathrm{~S}^{i \check{\bar{t}}} \otimes \mathscr{P}^{*} \overline{\mathscr{C}}^{\otimes \mathrm{D}}, \varphi_{\mathrm{D}}^{i}\right) \leqslant-\mathrm{D} \tau(i / \mathrm{D}) .
$$

The first two of these arithmetic estimates, (4.20) and (4.21), have been proven in Section 4.1 ((4.20) follows from Proposition 4.4, and (4.21) from (4.3), (4.5), and Lemma 4.3). We defer the proof of (4.22) to the next subsection.

To complete the proof of Theorem 3.4 taking Lemma 4.8 for granted, first observe that (4.21) and (4.23) imply that, for any $(\mathrm{D}, \imath) \in(\mathbf{N} \backslash\{0\}) \times \mathbf{N}$,

$$
\widehat{\mu}_{\max }\left(\mathrm{S}^{i} \check{\check{t}} \otimes \mathscr{P}^{*} \overline{\mathscr{D}}^{\otimes \mathrm{D}}\right)+h\left(\overline{\mathrm{E}}_{\mathrm{D}}^{i}, \mathrm{~S}^{i \check{t}} \otimes \mathscr{P}^{*} \overline{\mathscr{D}}^{\otimes \mathrm{D}}, \varphi_{\mathrm{D}}^{i}\right) \leqslant \mathrm{D}\left[c_{2} \frac{i}{\mathrm{D}}+c_{2}-\tau\left(\frac{i}{\mathrm{D}}\right)\right] .
$$

Together with (4.18) and (4.20), this shows that, for any $\mathrm{D} \in \mathbf{N} \backslash\{0\}$,

$$
\left(\mathrm{rkE}_{\mathrm{D}}\right)^{-1} \sum_{i \geqslant 0} \mathrm{rk}\left(\mathrm{E}_{\mathrm{D}}^{i} / \mathrm{E}_{\mathrm{D}}^{i+1}\right)\left[-c_{2} \frac{i}{\mathrm{D}}-c_{2}+\tau\left(\frac{i}{\mathrm{D}}\right)\right] \leqslant c_{1} \text {. }
$$

Suppose now that $v<d$. To derive a contradiction, let us choose a positive real number $\lambda$, and let $\lceil\lambda \mathrm{D}\rceil$ be the smallest integer $\geqslant \lambda \mathrm{D}$. By splitting the sum in (4.24) in two parts corresponding to $i<\lceil\lambda \mathrm{D}\rceil$ and to $i \geqslant\lceil\lambda \mathrm{D}\rceil$, we get:

$$
\left.(\operatorname{rk~E})_{D}\right)^{-1}\left[\operatorname{rk}\left(\mathrm{E}_{\mathrm{D}} / \mathrm{E}_{\mathrm{D}}^{\lceil\lambda \mathrm{D}\rceil}\right) \inf _{x \in \mathbf{R}_{+}}\left(\tau(x)-c_{2} x-c_{2}\right)+\operatorname{rk~E}_{\mathrm{D}}^{\lceil\lambda \mathrm{D}\rceil} \inf _{x \geqslant \lambda}\left(\tau(x)-c_{2} x-c_{2}\right)\right] \leqslant c_{1} .
$$

(Observe that the two inf's are finite by Lemma 4.8, 3).) According to Lemma 4.7, our assumption implies that, when $\mathrm{D}$ goes to infinity,

$$
\operatorname{rk}\left(\mathrm{E}_{\mathrm{D}}, \mathrm{E}_{\mathrm{D}}^{\lceil\lambda \mathrm{D}\rceil}\right)=o\left(\mathrm{D}^{d}\right)=o\left(\mathrm{rk} \mathrm{E}_{\mathrm{D}}\right)
$$

and

$$
\mathrm{rkE}_{\mathrm{D}}^{\lceil\lambda \mathrm{D}\rceil} \sim \mathrm{rkE}_{\mathrm{D}}
$$


Therefore,

$$
\inf _{x \geqslant \lambda}\left(\tau(x)-c_{2} x-c_{2}\right) \leqslant c_{1}
$$

Since $\lambda$ is arbitrary in $\mathbf{R}_{+}^{*}$, this contradicts (4.22) and completes the proof.

4.3. Upper bounds on the heights of evaluation maps

\subsubsection{Local bounds}

The last assertion in Lemma 4.8 will be a consequence of the following local estimates concerning the norms of the evaluation maps $\varphi_{\mathrm{D}}^{i}$.

Lemma 4.9. - For every $\mathbf{p}$ in $\mathrm{V}_{f}(\mathbf{K})$ and every $(\mathbf{D}, i) \in(\mathbf{N} \backslash\{0\}) \times \mathbf{N}$, the $\mathbf{p}$-adic norm of $\varphi_{\mathrm{D}}^{i}$ satisfies the following estimate:

$$
\left\|\varphi_{\mathrm{D}}^{i}\right\|_{\mathbf{p}} \leqslant \mathrm{R}_{\mathbf{p}}^{-i}
$$

Lemma 4.10 - For every embedding $\sigma: \mathrm{K} \hookrightarrow \mathbf{G}$, there exist $\mathrm{G}_{\sigma} \in[1,+\infty[$ and $\left.\left.\mathrm{R}_{\sigma} \in\right] 0,1\right]$ such that, for every $(\mathrm{D}, \imath) \in(\mathbf{N} \backslash\{0\}) \times \mathbf{N}$, the operator norm of $\varphi_{\mathrm{D}, \sigma}^{i}: \mathrm{E}_{\mathrm{D}, \sigma}^{i} \rightarrow$ $\mathrm{S}^{i} \check{t}_{\sigma} \otimes \mathrm{L}_{\mathrm{P}, \sigma}^{\otimes \mathrm{D}}$ satisfies the following estimate:

$$
\left\|\varphi_{\mathrm{D}, \sigma}^{i}\right\| \leqslant \mathrm{C}_{\sigma}^{\mathrm{D}} \mathrm{R}_{\sigma}^{-i}
$$

Lemma 4.11. - There exists a function $\tau_{0}: \mathbf{R}_{+} \rightarrow \mathbf{R}$, bounded from below, which satisfies

$$
\lim _{x \rightarrow+\infty} \frac{\tau_{0}(x)}{x}=+\infty
$$

and, for any $(\mathrm{D}, \imath) \in(\mathbf{N} \backslash\{0\}) \times \mathbf{N}$,

$$
\log \left\|\varphi_{\mathrm{D}, \sigma_{0}}^{i}\right\| \leqslant-\mathrm{D} \tau_{0}(i / \mathrm{D}) .
$$

Indeed, these three lemma imply the estimate (4.23), with $\tau$ the function defined by

$$
[\mathrm{K}: \mathrm{Q}] \tau(x):=\tau_{0}(x)-\alpha x-\beta,
$$

where $\alpha:=\sum_{\mathbf{p} \in \mathrm{V}_{f}(\mathrm{~K})} \log \mathrm{R}_{\mathbf{p}}^{-1}+\sum_{\sigma \neq \sigma_{0}} \log \mathrm{R}_{\sigma}^{-1}$ and $\beta:=\sum_{\sigma \neq \sigma_{0}} \log \mathrm{C}_{\sigma}$.

Lemma 4.9 is a consequence of Lemma 3.3.

The norm comparison estimates (4.8) and (4.9) show that, to prove Lemma 4.10 and 4.11 , we may as well define $\left\|\varphi_{\mathrm{D}, \sigma}^{i}\right\|$ as the operator norm of $\varphi_{\mathrm{D}, \sigma}^{i}: \mathrm{E}_{\mathrm{D}, \sigma}^{i} \rightarrow$ $\mathrm{S}^{i} \check{t}_{\sigma} \otimes \mathrm{L}_{\mathrm{P}, \sigma}^{\otimes \mathrm{D}}$ defined by using $\mathrm{L}^{\infty}$ norms, instead of $\mathrm{L}^{2}$ hermitian metrics, on the complex 
vector spaces $\mathrm{E}_{\mathrm{D}, \sigma}^{i}$. Then these lemma appear as special cases of Proposition 4.12 infra, of a purely analytic nature, to which the next two subsections are devoted.

\subsubsection{Archimedean bounds}

In this section, we consider a complex projective variety $\mathrm{X}$, a line bundle $\mathrm{L}$ over $\mathrm{X}$, a point $\mathrm{P}$ in $\mathrm{X}$, and a germ $\mathrm{V}$ of complex analytic submanifold of $\mathrm{X}(\mathbf{C})$ at $\mathrm{P}$ of dimension $d>0$. For any $(\mathrm{D}, \imath) \in(\mathbf{N} \backslash\{0\}) \times \mathbf{N}$, let $\mathrm{E}_{\mathrm{D}}^{i}$ be the subspace of $\Gamma\left(\mathrm{X}, \mathrm{L}^{\otimes \mathrm{D}}\right)$ formed by the regular sections of $\mathrm{L}^{\otimes \mathrm{D}}$ whose restriction to $\mathrm{V}$ vanish at order at least $i$ at $\mathrm{P}$, and let

$$
\varphi_{\mathrm{D}}^{i}: \mathrm{E}_{\mathrm{D}}^{i} \rightarrow \mathrm{S}^{i} \check{\mathrm{T}}_{\mathrm{P}} \mathrm{V} \otimes \mathrm{L}_{\mathrm{P}}^{\otimes \mathrm{D}}
$$

be the map which sends such a section to the jet of order $i$ of its restriction to $\mathrm{V}$.

Let us choose a continuous hermitian metric \|\|$_{\mathrm{L}}$ on $\mathrm{L}$ and a hermitian metric \|\|$_{\mathrm{T}}$ on the tangent space $\mathrm{T}_{\mathrm{P}} \mathrm{V}$ of $\mathrm{V}$ at $\mathrm{P}$. By taking tensor and symmetric powers, these

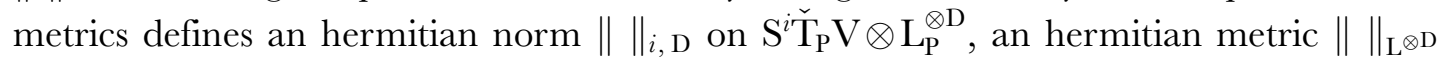
on $\mathrm{L}^{\otimes \mathrm{D}}$, and therefore an $\mathrm{L}^{\infty}$ norm \|\|$_{\mathrm{L}^{\infty}}: s \mapsto \max _{x \in \mathrm{X}(\mathrm{C})}\|s(x)\|_{\mathrm{L}^{\otimes \mathrm{D}}}$ on $\Gamma\left(\mathrm{X}, \mathrm{L}^{\otimes \mathrm{D}}\right)$ and its subspaces $\mathrm{E}_{\mathrm{D}}^{i}$. We shall denote by $\left\|\varphi_{\mathrm{D}}^{i}\right\|$ the operator norm of $\varphi_{\mathrm{D}}^{i}$ defined by means of these norms.

Proposition 4.12. - 1) There exist $\mathrm{G} \in[1,+\infty[$ and $\mathrm{R} \in] 0,1]$ such that

$$
\left\|\varphi_{\mathrm{D}}^{i}\right\| \leqslant \mathrm{C}^{\mathrm{D}} \mathrm{R}^{-i}
$$

2) If moreover there exists a complex manifold $\mathrm{M}$ satisfying the Liouville property, a point $\mathrm{O}$ of $\mathrm{M}$, and a holomorphic map $f: \mathrm{M} \rightarrow \mathrm{X}(\mathbf{C})$, which sends $\mathrm{O}$ to $\mathrm{P}$ and maps the germ of $\mathrm{M}$ at $\mathrm{O}$ biholomorphically onto $\mathrm{V}$, then

$$
\lim _{x \rightarrow+\infty} \sup _{\frac{i}{\mathrm{D}} \geqslant x} \frac{1}{i} \log \left\|\varphi_{\mathrm{D}}^{i}\right\|=-\infty .
$$

The validity of these two assertions is clearly independent of the choice of hermitian structures \|\|$_{\mathrm{L}}$ and \|\|$_{\mathrm{T}}$ on $\mathrm{L}$ and $\mathrm{T}_{\mathrm{P}} \mathrm{V}$.

The proof of Proposition 4.12 will use the following lemma, of independent interest.

Let $\lambda$ denote the Lebesgue measure on $\mathbf{G}^{d}$.

Lemma 4.13. - Let $f=\sum_{\mathrm{I} \in \mathbf{N}^{d}} a_{\mathrm{I}} z^{\mathrm{I}}$ be a holomorphic function on the open ball $\mathrm{B}(0, \mathrm{R})$ of center 0 and radius $\mathbf{R}>0$ in $\mathbf{C}^{d}$. Assume that $f$ vanishes at order exactly $i$ at 0 , and let $j^{i} f:=\sum_{|\mathrm{I}|=i} a_{\mathrm{I}} z^{\mathrm{I}}$ be the jet of order $i$ of $f$ at the origin. Then, if $\left\|j^{i} f\right\|_{\mathbf{S}^{i}\left(\check{\mathbf{C}}^{d}\right)}$ denotes the hermitian 
norm of $j^{i} f$ in the $i$-th symmetric power of the dual of $\mathbf{C}^{d}$ equipped with the standard hermitian metric, we have:

$$
\frac{1}{\lambda(\mathrm{B}(0, \mathrm{R}))} \int_{\mathrm{B}(0, \mathrm{R})} \log |f| d \lambda \geqslant \frac{1}{\lambda(\mathrm{B}(0, \mathrm{R}))} \int_{\mathrm{B}(0, \mathrm{R})} \log \left|j^{i} f\right| d \lambda
$$

$$
\geqslant \log \left\|j^{i} f\right\|_{\mathbf{S}^{i}\left(\check{\mathbf{C}}^{d}\right)}-\left(\log \mathrm{R}^{-1}\right) i-\alpha(d) i,
$$

where $\alpha(d)$ denotes a constant in $\mathbf{R}_{+}$depending only on $d$.

Observe that these inequalities implies the following variant of Cauchy's estimates:

$$
\log \left\|j^{i} f\right\|_{\mathbf{S}^{i}\left(\check{\mathbf{C}}^{d}\right)} \leqslant \log \sup _{x \in \mathbf{B}(0, \mathrm{R})}|f(x)|+\left(\log \mathrm{R}^{-1}\right) i+\alpha(d) i .
$$

Proof of Lemma 4.13. - If, for any $t \in \mathbf{C}$ such that $|t| \leqslant 1$, we let

$$
f_{t}(z):=\sum_{\mathrm{I} \in \mathbf{N}^{d}} a_{\mathrm{I}} t^{|\mathrm{I}|-i} z^{\mathrm{I}}
$$

then the integral

$$
\int_{\mathrm{B}(0, \mathrm{R})} \log \left|f_{t}\right| d \lambda
$$

- which is also equal to

$$
|t|^{-d} \int_{\mathrm{B}(0,|t| \mathrm{R})} \log |f| d \lambda-i \lambda(\mathrm{B}(0, \mathrm{R})) \log |t|
$$

when $t \neq 0-$ depends only on $|t|$ and is subharmonic on the open disk $\{t \in \mathbf{C}|| t \mid<1\}$. As $f_{0}=j^{i} f$ and $f_{1}=f$, this yields (4.28).

To prove (4.29), we use some computations in [BGS94]. With the notation of loc. cit. 1.4 .3 , with $\mathrm{V}=\mathbf{C}^{d}$ equipped with the standard hermitian metric, we get:

$$
\begin{aligned}
\frac{1}{\lambda(\mathrm{B}(0, \mathrm{R}))} \int_{\mathrm{B}(0, \mathrm{R})} \log \left|j^{i} f\right| d \lambda & =\log \left\|j^{i} f\right\|_{0}+\left(\int_{0}^{\mathrm{R}} r^{2 d-1} d r\right)^{-1} \int_{0}^{\mathrm{R}}\left(\log r^{i}\right) r^{2 d-1} d r \\
& =\log \left\|j^{i} f\right\|_{0}+i(\log \mathrm{R}-1 / 2 d) .
\end{aligned}
$$

Moreover, according to loc. cit., Corollary 1.4.3, (1.4.10), and Lemma 4.3.6, we have:

$$
\begin{aligned}
\log \left\|j^{i} f\right\|_{0} & \geqslant \log \left\|j^{i} f\right\|_{\infty}-\frac{i}{2} \sum_{m=1}^{d-1} \frac{1}{m} \\
& \geqslant \log \left\|j^{i} f\right\|_{2}-\frac{i}{2} \sum_{m=1}^{d-1} \frac{1}{m} \\
& \geqslant \log \left\|j^{i} f\right\|_{S^{i}\left(\check{\mathbf{C}}^{d}\right)}-\frac{1}{2} \log \left(\begin{array}{c}
d-1+i \\
i
\end{array}\right)-\frac{i}{2} \sum_{m=1}^{d-1} \frac{1}{m} .
\end{aligned}
$$

These estimates imply (4.29) with $\alpha(d)=1+\log d$. 
Proof of Proposition 4.12. - Assertion 1) follows from the variant (4.30) of Cauchy estimates, applied to the holomorphic functions defined by expressing holomorphic sections of $\mathrm{L}^{\otimes \mathrm{D}}$ on $\mathrm{V}$ in terms of some holomorphic trivialization of $\mathrm{L}$ on some holomorphic coordinate chart centered at $\mathrm{P}$.

To prove assertion 2), we assume that there exists a sequence $\left(\mathbf{D}_{n}, i_{n}, s_{n}\right)$ of triples, where $\mathbf{D}_{n} \in \mathbf{N} \backslash\{0\}, i_{n} \in \mathbf{N}$, and $s_{n} \in \mathbf{E}_{i_{n}}^{\mathrm{D}_{n}}$, and a constant $c \in \mathbf{R}$ satisfying

$$
\lim _{n \rightarrow+\infty} \frac{i_{n}}{\mathrm{D}_{n}}=+\infty
$$

and, for any $n, \frac{i_{n}}{\mathrm{D}_{n}} \geqslant 1,\left\|s_{n}\right\|_{\mathrm{L}^{\infty}} \leqslant 1$ and

$$
\log \frac{1}{i_{n}}\left\|\varphi_{\mathrm{D}_{n}}^{i_{n}}\left(s_{n}\right)\right\|_{i, \mathrm{D}} \geqslant-c .
$$

We are going to derive a contradiction, which will prove (4.27).

For any $n$, we let

$$
\rho_{n}:=\frac{1}{i_{n}} \log f^{*}\left\|s_{n}\right\|_{\mathrm{L}^{\otimes \mathrm{D}_{n}}}^{2} .
$$

It is a continuous locally integrable function from $\mathrm{M}$ to $[-\infty, 0]$. Let us assume - which is allowed - that the hermitian metric \|\|$_{\mathrm{L}}$ on $\mathrm{L}$ is $\mathrm{C}^{\infty}$, and let us denote by $\alpha$ the first Chern form of the holomorphic line bundle $f^{*} \mathrm{~L}$ equipped with the pull-back of this metric; it is a $\mathrm{C}^{\infty}$ closed $(1,1)$-form on $\mathrm{M}$. For any $n$, according to the Poincaré-Lelong equation, we have the equality of currents on $\mathrm{M}$ :

$$
d d^{c} \log f^{*}\left\|s_{n}\right\|_{\mathrm{L}^{\otimes \mathrm{D}_{n}}}^{2}=\delta_{\operatorname{div} f^{*} s_{n}}-\mathrm{D}_{n} \alpha .
$$

Therefore,

$$
d d^{c} \rho_{n} \geqslant-\frac{\mathrm{D}_{n}}{i_{n}} \alpha .
$$

Let $\Omega$ be the maximal open subset of M such that the sequence $\left(\rho_{n}\right)$ is bounded in $\mathrm{L}_{l o c}^{1}(\Omega)$. In other words, if $\mu$ denotes a $\mathrm{C}^{\infty}$ positive volume form on $\mathrm{M}$, a point $x$ of $\mathrm{M}$ belongs to $\Omega$ if and only if there exists an open neighbourhood $\mathrm{U}$ of $x$ and $c^{\prime} \in \mathbf{R}_{+}$ such that, for every $n$,

$$
\int_{\mathrm{U}} \rho_{n} \mu \geqslant-c^{\prime}
$$

Locally on $\mathrm{M}$, there exists a $\mathrm{C}^{\infty}$ real-valued function $\psi$ such that $\alpha=d d^{c} \psi$. Therefore, the functions $\rho_{n}+\psi$ are plurisubharmonic (hence subharmonic when expressed in 
any holomorphic coordinate chart) and bounded from above by $\psi$. This shows that $\Omega$ is a closed subset of M. Moreover, $\Omega$ is not empty; indeed, it contains the point $\mathrm{O}$, as follows from condition (4.31) and Lemma 4.13 applied to the holomorphic functions defined by expressing the holomorphic sections $f^{*} s_{n}$ of $f^{*} \mathrm{~L}^{\otimes \mathrm{D}_{n}}$ in terms of some holomorphic trivialization of $f^{*} \mathrm{~L}$ on some holomorphic coordinate chart centered at $\mathrm{O}$. As $\mathrm{M}$ is connected, this proves that $\Omega=\mathrm{M}$, i.e., that the sequence $\left(\rho_{n}\right)$ is bounded in $\mathrm{L}_{l o c}^{1}(\mathrm{M})$.

After possibly replacing the sequence $\left(\rho_{n}\right)$ by a subsequence, we may assume that it converges weakly to some current $\rho$ of order 0 on M. From (4.32), we get:

$$
d d^{c} \rho \geqslant 0
$$

and the current $\rho$ is therefore defined by a plurisubharmonic function on M. Moreover, as the functions $\rho_{n}$ are non-positive, the plurisubharmonic function $\rho$ is non-positive too, hence constant, since $\mathrm{M}$ satisfies the Liouville property.

On the other hand, we may consider the blow-up $v: \widetilde{M} \rightarrow \mathrm{M}$ of $\mathrm{O}$ in $\mathrm{M}$. Let $\mathrm{E}:=\mathrm{v}^{-1}(\mathrm{O})$ be its exceptional divisor, and define

$$
\widetilde{\rho}_{n}:=v^{*} \rho_{n}=\frac{1}{i_{n}} \log (f \circ \mathrm{v})^{*}\left\|s_{n}\right\|^{2} .
$$

From (4.32), we get:

$$
d d^{c} \widetilde{\rho}_{n} \geqslant-\frac{\mathrm{D}_{n}}{i_{n}} v^{*} \alpha .
$$

The same reasoning as above, applied to the sequence $\left(\widetilde{\rho}_{n}\right)$ instead of $\left(\boldsymbol{\rho}_{n}\right)$, shows that the maximal open subset $\widetilde{\Omega}$ of $\widetilde{M}$ such that $\left(\widetilde{\rho}_{n}\right)$ is bounded in $\mathrm{L}_{l o c}^{1}(\widetilde{\Omega})$ is closed. Moreover, it is non-empty (it contains $\widetilde{\mathrm{M}} \backslash \mathrm{E}$, which is mapped biholomorphically onto $\mathrm{M} \backslash\{\mathrm{O}\}$ by $v$ ), hence coincides with the connected manifold $\widetilde{\mathrm{M}}$. Finally, we obtain that the sequence $\left(\widetilde{\boldsymbol{\rho}}_{n}\right)$ is bounded in $\mathrm{L}_{l o c}^{1}(\widetilde{\mathrm{M}})$, and we may assume that it converges weakly to some current $\widetilde{\rho}$, defined by some plurisubharmonic function on $\widetilde{M}$.

Since $(f \circ \mathrm{v})^{*} s_{n}$ vanishes at order $i_{n}$ along $\mathrm{E}$, we also have

$$
d d^{c} \widetilde{\rho}_{n} \geqslant \delta_{\mathrm{E}}-\frac{\mathrm{D}_{n}}{i_{n}} v^{*} \alpha
$$

Therefore,

$$
d d^{c} \widetilde{\rho} \geqslant \delta_{\mathrm{E}}
$$


In particular, the locally integrable function $\widetilde{\rho}$ is not constant on $\widetilde{M}$, hence not constant on $\tilde{\mathrm{M}} \backslash$ E. This contradicts the fact that, on this open subset, it coincides with $v^{*} \rho$, which is constant.

It should be observed that the projectivity assumption on $\mathrm{X}$ plays basically no role in the proof of Proposition 4.12: indeed, this proposition still holds, with the same proof, when $\mathrm{X}$ is any complex reduced analytic space, equipped with a holomorphic line bundle endowed with a continuous hermitian metric, provided we now define $\mathrm{E}_{\mathrm{D}}^{i}$ as the subspace of bounded holomorphic sections of $\mathrm{L}^{\otimes \mathrm{D}}$ whose restriction to $\mathrm{V}$ vanish at order at least $i$ at P. (To prove assertion 2) of this generalization of Proposition 4.12, one immediately reduces by pull-back to the case where $\mathrm{X}=\mathrm{M}$, which basically has been handled in the proof above.)

\subsubsection{A variation on the First Main Theorem of value distribution theory}

In this final section, we want to discuss another proof of Proposition 4.12, in the special case where $\mathrm{M}$ is an affine space $\mathbf{C}^{n}$ and $\mathrm{L}$ is ample. (This special case of Proposition 4.12 would be enough to complete the proof of Theorem 3.4 when $\mathbf{M}=\mathbf{C}^{n}$ and $\mathbf{X}$ is quasiprojective, and therefore to establish Theorem 2.3.)

Observe first that Proposition 4.12 could be rephrased as the existence, for every $\rho \in \mathbf{R}_{+}^{*}$, of some constant $c(\rho) \in \mathbf{R}$ such that, for any $(\mathbf{D}, \imath) \in(\mathbf{N} \backslash\{0\}) \times \mathbf{N}$,

$$
\log \left\|\varphi_{\mathrm{D}}^{i}\right\| \leqslant-\rho i+\mathrm{D} c(\rho)
$$

In the special case where $\mathbf{M}=\mathbf{C}^{n}$, this easily follows from the Schwarz Lemma for holomorphic functions on $\mathbf{G}^{n}$ vanishing with order $i$ at the origin, applied on the ball of radius $\mathrm{R}=e^{\rho}$ (see for instance [CB01], 4.7.6).

When moreover $\mathrm{L}$ is ample, it is possible to give a more elaborated version of this argument, which has the advantage of relating the function $\tau$ controlling the norms $\left\|\varphi_{\mathrm{D}}^{i}\right\|$ (as in Lemma 4.11) to the growth of the map $f: \mathbf{M} \rightarrow \mathbf{X}(\mathbf{G})$. It relies on a refined variant of the First Main Theorem of higher dimensional Nevanlinna theory, and, hopefully, should clarify the origin of the "smallness" of the evaluation map asserted by Proposition 4.12, 2). We refer the reader to [GK73], [Sto77], or [Sha85] for expositions and references concerning higher dimensional Nevanlinna theory.

Let us consider a complex projective variety $\mathrm{X}$, an ample line bundle $\mathrm{L}$ over $\mathrm{X}$, a $\mathrm{C}^{\infty}$ hermitian metric \|\|$_{\mathrm{L}}$ on $\mathrm{L}$, whose first Chern form $\eta$ is positive, an integer $n \geqslant 1$, and a holomorphic $\operatorname{map} f: \mathbf{G}^{n} \rightarrow \mathbf{X}(\mathbf{G})$.

Let $\omega$ denote the positive $(1,1)$-form on $\mathbf{C}^{n} \backslash\{0\}$ defined by $\omega:=d d^{c} \log \|z\|^{2}$, where \|\| denotes the standard hermitian norm on $\mathbf{C}^{n}$, defined by $\left\|\left(z_{i}\right)_{1 \leqslant i \leqslant n}\right\|^{2}=\sum_{i=1}^{n}\left|z_{i}\right|^{2}$. This form may also be defined as the pull-back by the projection $\mathbf{C}^{n} \backslash\{0\} \rightarrow \mathbf{P}^{n-1}(\mathbf{G})$ of the standard Fubini-Study $(1,1)$-form on $\mathbf{P}^{n-1}(\mathbf{G})$. For any $r \in \mathbf{R}_{+}^{*}$, we also let 
$\mathrm{B}(r):=\left\{z \in \mathbf{G}^{\mathrm{N}} \mid\|z\|<r\right\}$ and we define $\mu_{r}$ as the unique probability measure on the sphere $\partial \mathrm{B}(r)$ of radius $r$ in $\mathbf{C}^{n}$, invariant under the unitary group $\mathrm{U}(n)$.

The growth of the holomorphic map $f$, relative to the hermitian line bundle $\overline{\mathrm{L}}:=\left(\mathrm{L},\|\|_{\mathrm{L}}\right)$ on $\mathrm{X}(\mathbf{G})$, is measured by the so-called order or characteristic function $\mathrm{T}_{f, \overline{\mathrm{L}}}: \mathbf{R}_{+}^{*} \rightarrow \mathbf{R}_{+}$, defined by

$$
\mathrm{T}_{f, \overline{\mathrm{L}}}(r):=\int_{\mathbf{C}^{n} \backslash\{0\}} \log ^{+} \frac{r}{\|z\|} \omega^{n-1} \wedge f^{*} \eta=\int_{0}^{r}\left(\int_{\mathrm{B}(t)} \omega^{n-1} \wedge f^{*} \eta\right) \frac{d t}{t} .
$$

The order function is a non-decreasing convex function of $\log r$. It is unbounded as soon as $f$ is non-constant; moreover, it is $\mathrm{O}(\log r)$ when $r$ goes to infinity if and only if the map $f$ is algebraic (see [GK73], Proposition 5.9, or [Sha85], Chapter II, Theorem $5)$.

Let $\mathrm{D}$ be a positive integer and let $s$ be a regular section of $\mathrm{L}^{\otimes \mathrm{D}}$ over $\mathrm{X}$, such that $f\left(\mathbf{C}^{n}\right)$ is not included in $|\operatorname{div} s|(\mathbf{C})$. Then $f^{*} \operatorname{div} s=\operatorname{div} f^{*} s$ is a well defined analytic divisor in $\mathbf{C}^{n}$. Its growth is measured by the so-called counting function of $r \in \mathbf{R}_{+}^{*}$ :

$$
\mathbf{N}_{f}(\operatorname{div} s, r):=\int_{\mathbf{C}^{n} \backslash\{0\}} \log ^{+} \frac{r}{\|z\|} \omega^{n-1} \boldsymbol{\delta}_{f^{*} \operatorname{div} s}=\int_{0}^{r}\left(\int_{\mathbf{B}(t)} \omega^{n-1} \boldsymbol{\delta}_{f^{*} \operatorname{div} s}\right) \frac{d t}{t} .
$$

Let $i$ be the order of vanishing of $f^{*} s$ at $0 \in \mathbf{C}^{n}$, and let $j^{i} f^{*} s$ be its jet of order $i$ at 0 . This jet may be seen as an element of $\mathrm{S}^{i} \check{\mathbf{C}}^{n} \otimes \mathrm{L}_{\mathrm{P}}^{\otimes \mathrm{D}}$, and, as such, has a well defined hermitian norm $\left\|j^{i} f^{*} s\right\|_{i, \mathrm{D}}$ defined by taking dual, tensor and symmetric powers from the standard hermitian norm on $\mathbf{C}^{n}$ and the given norm \|\|$_{\mathrm{L}}$ on $\mathrm{L}_{\mathrm{P}}$. The jet $j^{i} f^{*} s$ may also be seen as a homogeneous map of degree $\mathrm{D}$ from $\mathbf{G}^{n}$ to $\mathrm{L}_{\mathrm{P}}^{\otimes \mathrm{D}}$, and, as such, has a "quasi-norm" $\left\|j^{i} f^{*}\right\|_{0}$ defined by

$$
\log \left\|j^{i} f^{*} s\right\|_{0}:=\int_{\partial \mathrm{B}(1)} \log \left\|j^{i} f^{*} s(z)\right\|_{\mathrm{L}_{\mathrm{P}} \mathrm{D}} d \mu_{1}(z) .
$$

The norms $\left\|j^{i} f^{*} s\right\|_{i, \mathrm{D}}$ and $\left\|j^{i} f^{*} s\right\|_{0}$ are related by the following inequalities (cf. [BGS94], Corollary 1.4.3, (1.4.10), and Lemma 4.3.6):

$$
\log \left\|j^{i} f^{*} s\right\|_{0} \leqslant \log \left\|j^{i} f^{*} s\right\|_{i, \mathrm{D}}-\frac{1}{2} \log \left(\begin{array}{c}
n-1+i \\
i
\end{array}\right) \leqslant \log \left\|j^{i} f^{*} s\right\|_{0}+\frac{i}{2} \sum_{m=1}^{n-1} \frac{1}{m} .
$$

As a consequence, we get the following estimate:

$$
\log \left\|j^{i} f^{*} s\right\|_{i, \mathrm{D}} \leqslant \log \left\|j^{i} f^{*} s\right\|_{0}+c_{n} i,
$$

where $c_{n}:=\frac{1}{2}+\log n$.

We are now in position to state our refined version of the First Main Theorem of Nevanlinna theory - usually, it is stated as an equality only up to a bounded term, valid when $i=0$ (i.e., for sections $s$ which do not vanish at $f(0)$ ). 
Proposition 4.14. - With the above notation, for every $r \in \mathbf{R}_{+}^{*}$, we have

$$
\log \left\|j^{i} f^{*} s\right\|_{0}=\int_{\partial \mathrm{B}(r)} \log f^{*}\|s\|_{\overline{\mathrm{L}}^{\otimes \mathrm{D}}} d \mu_{r}-i \log r-\mathrm{N}_{f}(\operatorname{div} s, r)+\mathrm{DT}_{f, \overline{\mathrm{L}}(r) .}
$$

Seen as a function of $r$, the integral

$$
\int_{\partial \mathrm{B}(r)} \log f^{*}\|s\|_{\overline{\mathrm{L}}^{\otimes \mathrm{D}}}^{-1} d \mu_{r}
$$

is classically called the proximity function, relative to $f$, associated to the $\operatorname{divisor} \operatorname{div} s$, and denoted $m_{f}(\operatorname{div} s, r)$. The traditional formulation of the First Main Theorem is the following consequence of (4.34) specialized to $\mathrm{D}=1$ and $i=0$ :

$$
\mathrm{N}_{f}(\operatorname{div} s, r)+m_{f}(\operatorname{div} s, r)=\mathrm{T}_{f, \overline{\mathrm{L}}}(r)+\mathrm{O}(1) .
$$

Sketch of proof of Proposition 4.14. - Let us introduce the blow-up $v: \widetilde{\mathbf{C}}^{n} \rightarrow \mathbf{C}^{n}$ of $0 \in \mathbf{G}^{n}$ and let $\mathrm{E}:=\boldsymbol{v}^{-1}(0)$ be its exceptional divisor. We shall freely identify $\mathbf{C}^{n} \backslash\{0\}$ and $\widetilde{\mathbf{C}}^{n} \backslash$ E. The projection $\mathbf{G}^{n} \backslash\{0\} \rightarrow \mathbf{P}^{n-1}(\mathbf{C})$ composed with $\boldsymbol{v}$ extends to a morphism $p: \widetilde{\mathbf{C}}^{n} \rightarrow \mathbf{P}^{n-1}(\mathbf{G})$, and $p_{\mid \mathrm{E}}: \mathrm{E} \rightarrow \mathbf{P}^{n-1}(\mathbf{G})$ is an isomorphism. In consequence, the form $\omega$ on $\mathbf{C}^{n} \backslash\{0\} \simeq \widetilde{\mathbf{C}}^{n} \backslash \mathrm{E}$ extends to a $\mathrm{C}^{\infty}$ form on $\widetilde{\mathbf{C}}^{n}$, which we shall still denote $\boldsymbol{\omega}$; it is the pull-back by $p$ of the Fubini-Study form on $\mathbf{P}^{n-1}(\mathbf{C})$, and satisfies:

$$
\omega^{n}=0
$$

and

$$
\int_{\mathrm{E}} \omega^{n-1}=1
$$

The equality (4.34) will follow by integration from the following local identities relating currents on $\widetilde{\mathbf{C}}^{n}$ :

Lemma 4.15. - Let $r$ be a positive real number.

1) There exists a current $\mathrm{C}_{r}$ of bidegree $(1,1)$, of order 0 , supported by $\partial \mathrm{B}(r)$ and $\mathrm{U}(n)$ invariant, such that the following identity of currents on $\widetilde{\mathbf{C}}^{n}$ holds:

$$
d d^{c} \mathbf{v}^{*} \log ^{+} \frac{r^{2}}{\|z\|^{2}}=\omega \mathbf{1}_{v^{-1} \mathrm{~B}(r)}-\boldsymbol{\delta}_{\mathrm{E}}+\mathrm{C}_{r} .
$$

2) Moreover, we have the following equality of currents of bidegree $(n, n)$ on $\widetilde{\mathbf{C}}^{n}$ :

$$
d d^{c}\left(\omega^{n-1} v^{*} \log ^{+} \frac{r^{2}}{\|z\|^{2}}\right)=-\omega^{n-1} \delta_{\mathrm{E}}+\mu_{r} .
$$


Proof. - To prove (4.37), we write

$$
v^{*} \log ^{+} \frac{r^{2}}{\|z\|^{2}}=\mathbf{1}_{\mathrm{v}^{-1} \mathrm{~B}(r)} \mathbf{v}^{*} \log \frac{r^{2}}{\|z\|^{2}}
$$

and we observe that $v^{*} \log \frac{r^{2}}{\|z\|^{2}}$ satisfies the Poincaré-Lelong equation

$$
d d^{c} \boldsymbol{v}^{*} \log \frac{r^{2}}{\|z\|^{2}}=\omega-\delta_{\mathrm{E}}
$$

and vanishes on $\partial \mathrm{B}(r)$. This entails (4.37) with

$$
\mathrm{C}_{r}=d \boldsymbol{v}^{*} \log \frac{r^{2}}{\|z\|^{2}} \wedge d^{c} \mathbf{1}_{v^{-1} \mathrm{~B}(r)} .
$$

From (4.37), we get:

$$
\begin{aligned}
d d^{c}\left(\omega^{n-1} v^{*} \log ^{+} \frac{r^{2}}{\|z\|^{2}}\right) & =\omega^{n-1} d d^{c} v^{*} \log ^{+} \frac{r^{2}}{\|z\|^{2}} \\
& =\omega^{n} \mathbf{1}_{v^{-1} \mathrm{~B}(r)}-\omega^{n-1} \delta_{\mathrm{E}}+\omega^{n-1} C_{r} .
\end{aligned}
$$

Using (4.35), this leads to (4.38) with $\omega^{n-1} \mathrm{C}_{r}$ instead of $\mu_{r}$. Finally $\omega^{n-1} \mathrm{C}_{r}$ is a current of degree 0 and type $(n, n)$ supported by $\partial \mathrm{B}(r)$ - in other words a measure on $\partial \mathrm{B}(r)$ which is $\mathrm{U}(n)$-invariant, and has the same class in $\mathrm{H}_{c}^{2 n}\left(\widetilde{\mathbf{C}}^{n}, \mathbf{R}\right)$ as $\omega^{n-1} \delta_{\mathrm{E}}$, and therefore has total mass 1 , by (4.36). Since these properties characterize $\boldsymbol{\mu}_{r}$, this completes the proof of (4.38).

Consider now a section $s$ of $\mathrm{L}^{\otimes \mathrm{D}}$ over $\mathrm{X}$, as in the statement of Proposition 4.14. Its pull-back $(f \circ \boldsymbol{v})^{*} s$ on $\widetilde{\mathbf{C}}^{n}$ defines a holomorphic section of $(f \circ \mathbf{v})^{*} \mathrm{~L}^{\otimes \mathrm{D}}(-i \mathrm{E})$, which is not identically 0 on E. Therefore, the divisor of singularities of

$$
\varphi:=\log (f \circ v)^{*}\|s\|^{2}-i \log \|z\|^{2}
$$

does not contain E. Moreover, the restriction of $\varphi$ to $\mathrm{E}$, identified with $\mathbf{P}^{n-1}(\mathbf{C})$, coincides with the map

$$
\left(z_{1}: \ldots: z_{n}\right) \longmapsto \log \frac{\left\|j^{i} f^{*} s\left(z_{1}, \ldots, z_{n}\right)\right\|_{\mathrm{L}_{\mathrm{P}}^{\otimes \mathrm{D}}}^{2}}{\left\|\left(z_{1}, \ldots, z_{n}\right)\right\|^{2 i}} .
$$

Therefore,

$$
\int_{\widetilde{\mathrm{C}}^{n}} \varphi \omega^{n-1} \delta_{\mathrm{E}}=\log \left\|j^{i} f^{*} s\right\|_{0}
$$


Moreover, from the Poincaré-Lelong equation, we also get:

$$
d d^{c} \boldsymbol{\varphi}=\delta_{\left(f^{*} \operatorname{div} s\right)}-\mathrm{D}(f \circ v)^{*} \eta+i \omega,
$$

where $\left(f^{*} \operatorname{div} s\right)^{\sim}:=(f \circ \boldsymbol{v})^{*} \operatorname{div} s-i \mathrm{E}$ denotes the proper transform of $f^{*} \operatorname{div} s$ under $\boldsymbol{v}$.

The identity (4.34) finally follows from Stokes formula (13)

$$
\int_{\widetilde{\mathbf{C}}^{n}} \varphi d d^{c}\left(\omega^{n-1} \boldsymbol{v}^{*} \log ^{+} \frac{r^{2}}{\|z\|^{2}}\right)=\int_{\widetilde{\mathbf{C}}^{n}}\left(d d^{c} \varphi\right) \omega^{n-1} \boldsymbol{v}^{*} \log ^{+} \frac{r^{2}}{\|z\|^{2}},
$$

combined with (4.38), (4.39), and (4.40).

From Proposition 4.14, we immediately derive, taking into account the nonnegativity of the counting function and the estimate (4.33):

Corollary 4.16. - With the above notation, we have:

$$
\log \left\|j^{i} f^{*} s\right\|_{i, \mathrm{D}} \leqslant \log \|s\|_{\mathrm{L}^{\infty}}-i \log r+\mathrm{DT}_{f, \overline{\mathrm{L}}}(r)+c_{n} i,
$$

where

$$
\|s\|_{\mathrm{L}^{\infty}}:=\max _{x \in \mathrm{X}(\mathbf{C})}\|s(x)\|_{\overline{\mathrm{L}}^{\otimes D}}
$$

The estimate (4.41) means that the operator norm $\left\|\varphi_{\mathrm{D}}^{i}\right\|$ of the map $\varphi_{\mathrm{D}}^{i}$ which sends an element $s$ of the space

$$
\mathrm{E}_{\mathrm{D}}^{i}:=\left\{s \in \Gamma\left(\mathrm{X}, \mathrm{L}^{\otimes \mathrm{D}}\right) \mid f^{*} s \text { vanishes at order at least } i \text { at } 0\right\},
$$

equipped with the norm \|\|$_{\mathrm{L}^{\infty}}$, to the jet $j^{i} f^{*} s$ in the space $\mathrm{S}^{i} \check{\mathbf{C}}^{n} \otimes \mathrm{L}_{\mathrm{P}}^{\otimes \mathrm{D}}$ equipped with the norm \|\|$_{i, \mathrm{D}}$, satisfies the following upper bound:

$$
\frac{1}{\mathrm{D}} \log \left\|\varphi_{\mathrm{D}}^{i}\right\| \leqslant-\frac{i}{\mathrm{D}} \log r+\mathrm{T}_{f, \overline{\mathrm{L}}}(r)+c_{n} \frac{i}{\mathrm{D}}
$$

Therefore, if $\widetilde{\mathrm{T}}_{f, \overline{\mathrm{L}}}: \mathbf{R}_{+} \rightarrow \mathbf{R} \cup\{+\infty\}$ denotes the Legendre transform of the convex function $\mathrm{T}_{f, \overline{\mathrm{L}}}(r)$ of $\log r$, defined by

$$
\widetilde{\mathrm{T}}_{f, \overline{\mathrm{L}}}(\xi):=\sup _{r \in \mathbf{R}_{+}^{*}}\left(\xi \log r-\mathrm{T}_{f, \overline{\mathrm{L}}}(r)\right),
$$

\footnotetext{
(13) Standard techniques allow to apply Stokes formula to the currents $\varphi$ and $\omega^{n-1} v^{*} \log +\frac{r^{2}}{\|z\|^{2}}$, which are not of class $\mathrm{C}^{2}$, but have "gentle enough" singularities. See for instance [GK73], 1(a), or [BGS94], 1.3 5).
} 
then we have:

$$
\frac{1}{\mathrm{D}} \log \left\|\varphi_{\mathrm{D}}^{i}\right\| \leqslant-\widetilde{\mathrm{T}}_{f, \overline{\mathrm{L}}}(i / \mathrm{D})+c_{n} i / \mathrm{D}
$$

Since the function $\widetilde{\mathrm{T}}_{f, \overline{\mathrm{L}}}-$ like the Legendre transform of any convex function on $\mathbf{R}-$ is bounded below and satisfies

$$
\lim _{\xi \rightarrow+\infty} \widetilde{\mathrm{T}}_{f, \overline{\mathrm{L}}}(\xi) / \xi=+\infty
$$

this estimate, applied to the situation where $f$ is an immersion at the origin, provides another proof of Proposition 4.12 when $\mathrm{M}$ is an affine space $\mathbf{G}^{n}$ and $\mathrm{L}$ is ample.

Actually, we may distinguish two cases:

1. The holomorphic map $f$ is algebraic, or equivalently, $\mathrm{G}:=\lim _{r \rightarrow+\infty}(\log r)^{-1} \mathrm{~T}_{f, \overline{\mathrm{L}}}(r)$ is

finite. Then, for any $\xi>\mathrm{C}, \widetilde{\mathrm{T}}_{f, \overline{\mathrm{L}}}(\xi)=+\infty$. This shows that $\left\|\varphi_{\mathrm{D}}^{i}\right\|$, and therefore $\mathrm{E}_{\mathrm{D}}^{i}$, vanishes for $i / \mathrm{D}>\mathrm{C}$.

2. The holomorphic map $f$ is not algebraic, or equivalently, $\lim _{r \rightarrow+\infty}(\log r)^{-1} \mathrm{~T}_{f, \overline{\mathrm{L}}}(r)=$ $+\infty$. Then $\widetilde{\mathrm{T}}_{f, \overline{\mathrm{L}}}$ is a real valued convex function on $\mathbf{R}_{+}$.

Finally, let us indicate that standard techniques (see for instance [Shi75]) allow to extend this proof to the situation where $f$ is only assumed to be meromorphic from $\mathbf{C}^{n}$ to $\mathbf{M}$, and holomorphic on a neighbourhood of 0 . This generalization would be sufficient to prove Theorem 3.4 when $\mathrm{M}$ is the complement of some closed analytic subset in $\mathbf{G}^{n}$ and $f: \mathbf{G}^{n} \rightarrow \mathbf{X}_{\sigma_{0}}(\mathbf{G})$ is meromorphic.

\section{Appendix: smooth principal bundles and connections}

In this appendix, we recall a few elementary facts concerning principal bundles and connections in the algebraic setting. Although these facts are clearly well-known, it appears difficult to point out a source presenting them systematically.

Concerning the definition and basic operations on principal G-bundles, the famous paper [Ser58] by Serre - where étale localization is introduced in algebraic geometry - probably remains the best reference; see also [Gro71], Section XI.4. Observe also that definitions of G-bundles of various degrees of generality occur in the literature; the one we recall below - which involves only smooth data - is a rather restrictive one.

Concerning connections and their curvatures, the algebraic theory is a paraphrase of the classical $\mathrm{C}^{\infty}$ theory, as presented for instance in [KN63], Chapter II. In characteristic $p>0$, the construction and properties of the $p$-curvature of a flat connection described below are straightforward consequences of definitions and of basic properties of the $p$-curvature of involutive subbundles of the tangent bundle of a smooth variety (cf. [Kat70] and [Eke87]). 


\section{A.1. Basic definitions}

Let $\mathrm{G}$ be a smooth algebraic group over some field $\mathrm{K}$. By definition, a principal G-bundle over a smooth $\mathrm{K}$-scheme $\mathrm{B}$ is a smooth $\mathrm{K}$-scheme $\mathrm{X}$ equipped with a rightaction of $\mathrm{G}, \alpha: \mathrm{X} \times{ }_{\mathrm{K}} \mathrm{G} \rightarrow \mathrm{X}$ and a K-morphism $q: \mathrm{X} \rightarrow \mathrm{B}$ which is G-invariant (that is, $q \circ \alpha=p r_{1}$ ) and satisfies the following equivalent conditions ${ }^{(14)}$ :

1. The morphism $q$ is surjective and smooth and $\left(p r_{1}, \alpha\right): \mathrm{X} \times{ }_{\mathrm{K}} \mathrm{G} \rightarrow \mathrm{X} \times_{\mathrm{B}} \mathrm{X}$ is an isomorphism.

2. There exists a surjective étale morphism of schemes $\mathrm{B}^{\prime} \rightarrow \mathrm{B}$ such that the $\mathrm{G}$-space $\mathrm{X}^{\prime}:=\mathrm{B}^{\prime} \times_{\mathrm{B}} \mathrm{X}$ is isomorphic to the trivial right $\mathrm{G}$-space $\mathrm{B}^{\prime} \times_{\mathrm{K}} \mathrm{G}$ over $\mathrm{B}^{\prime}$.

For any such principal G-bundle, we may consider the canonical short exact sequence of G-equivariant vector bundles over $\mathrm{X}$ :

$$
\mathrm{O} \rightarrow \mathrm{T}_{q} \rightarrow \mathrm{T}_{\mathrm{X}} \rightarrow q^{*} \mathrm{~T}_{\mathrm{B}} \rightarrow 0
$$

Moreover, the G-equivariant bundle $\mathrm{T}_{q}$ over $\mathrm{X}$ is canonically isomorphic to the trivial vector bundle of fiber Lie $\mathrm{G}$, endowed with the adjoint action of $\mathrm{G}$, and G-invariant regular sections of $\mathrm{T}_{q}$ define a locally free coherent sheaf on $\mathrm{B}$, and may be identified with the sheaf of regular sections of a vector bundle $\operatorname{Ad}(\mathrm{X})$ on $\mathrm{B}$ (which indeed is the vector bundle deduced from the G-bundle $\mathrm{X}$ by means of the adjoint representation $\mathrm{G} \rightarrow \mathrm{GL}(\mathrm{Lie} G))$.

By definition, a connection on the G-bundle $q: \mathrm{X} \rightarrow \mathrm{B}$ is a G-equivariant splitting of (A.1) or, equivalently, a G-equivariant subvector bundle $\mathrm{H}$ of $\mathrm{T}_{\mathrm{X}}$ such that $\mathrm{T}_{\mathrm{X}}=\mathrm{T}_{q} \oplus \mathrm{H}$. The associated connection form $\theta_{\mathrm{H}}$ is the section of $\Omega_{\mathrm{X}}^{1} \otimes \mathrm{T}_{q}$ defined by the projection from $\mathrm{T}_{\mathrm{X}}$ onto $\mathrm{T}_{q}$ with kernel $\mathrm{H}$. over X

For any such connection $\mathrm{H}$, we may consider the morphism of vector bundles

$$
\Lambda^{2} \mathrm{H} \longrightarrow \mathrm{T}_{\mathrm{X}} / \mathrm{H}
$$

which, for any two local sections $v_{1}$ and $v_{2}$ of $\mathrm{H}$, maps $v_{1} \wedge v_{2}$ to the class of their Lie bracket $\left[v_{1}, v_{2}\right]$. It is G-equivariant; therefore, as we have canonical isomorphisms of G-equivariant vector bundles

$$
\left(\Lambda^{2} \mathrm{H}\right)^{`} \simeq q^{*} \Omega_{\mathrm{B}}^{2}
$$

and

$$
\mathrm{T}_{\mathrm{X}} / \mathrm{H} \simeq \mathrm{T}_{q}
$$

(14) These conditions mean that the G-torsor X over B is locally trivial in the étale topology; we would get an equivalent definition by working with the flat topology. 
it may be identified with a regular section on $\mathrm{B}$ of the vector bundle $\Omega_{\mathrm{B}}^{2} \otimes A d(\mathrm{X})$, the curvature of the G-bundle with connection $(\mathrm{X}, \mathrm{H})$. When this curvature vanishes - or, equivalently, when $\mathrm{H}$ is involutive - the connection $\mathrm{H}$ is said to be flat.

Similarly, when the connection $\mathrm{H}$ is flat and $\mathrm{K}$ is a field of characteristic $p>0$, then the $p$-curvature of the involutive bundle $\mathrm{H}$ ( $c f$. section 2.1.1), which is a G-invariant regular section of $F r_{0} b^{*} \check{\mathrm{H}} \otimes \mathrm{T}_{\mathrm{X}} / \mathrm{H}$ over $\mathrm{X}$, gets identified with a regular section of Frob ${ }^{*} \Omega_{\mathrm{B}}^{1} \otimes A d(\mathrm{X})$, the so-called p-curvature of the flat connection $\mathrm{H}$ on the $\mathrm{G}$-bundle $\mathrm{X}$.

When the base field $\mathrm{K}$ is a number field, for any algebraic group $\mathrm{G}$ over $\mathrm{K}$ and any principal G-bundle $\mathrm{X}$ over a smooth base $\mathrm{B}$ endowed with a flat connection $\mathrm{H}$ as above, we may consider smooth models $\mathscr{G}, \mathscr{B} ; \mathscr{B}, \mathscr{H}, \ldots$ of $\mathrm{G}, \mathrm{X}, \mathrm{B}, \mathrm{H}, \ldots$ over Spec $\mathscr{O}_{\mathrm{K}}[1 / \mathrm{N}]$, for $\mathrm{N}$ a sufficiently divisible integer. Then, for any prime ideal $\mathbf{p}$ of $\mathscr{C}_{\mathrm{K}}$ not dividing $\mathrm{N}$, by reducing modulo $\mathbf{p}$, we get a principal $\mathscr{G}_{\mathbf{F}_{\mathrm{p}}}$-bundle $\mathscr{C}_{\mathbf{F}_{\mathrm{p}}}$ over $\mathscr{B}_{\mathbf{F}_{\mathrm{p}}}$, equipped with the flat connection $\mathscr{H}_{\mathbf{F}_{\mathrm{p}}}$. Moreover, the vanishing of the $p$-curvature of these flat principal bundles for almost every $\mathbf{p}$ does not depend on the choice of these models.

\section{A.2. Constructions of smooth principal G-bundles and connections}

As in the preceding section, we denote by $\mathrm{X}$ a smooth principal G-bundle over some smooth base $\mathrm{B}$ over a field $\mathrm{K}$, and by $p: \mathrm{X} \rightarrow \mathrm{B}$ the structural morphism of this bundle.

Inverse image. - If $\mathrm{B}^{\prime}$ is another smooth $\mathrm{K}$-scheme and $f: \mathrm{B}^{\prime} \rightarrow \mathrm{B}$ is a $\mathrm{K}$ morphism, then we can form the following cartesian diagram of K-schemes

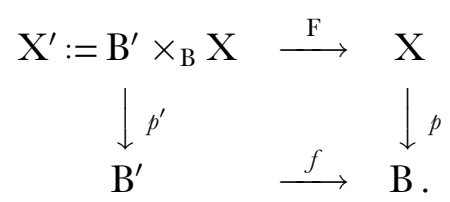

Then $\mathrm{X}^{\prime}$ is naturally equipped with a right-action of $\mathrm{G}$, the $\mathrm{K}$-morphism $p^{\prime}: \mathrm{X}^{\prime} \rightarrow \mathrm{B}^{\prime}$ is smooth and defines a principal G-bundle $\mathrm{X}^{\prime}$ with base $\mathrm{B}^{\prime}$. Moreover, the differential of $\mathrm{F}$ defines an isomorphism

$$
\mathrm{T}_{p^{\prime}} \simeq \mathrm{F}^{*} \mathrm{~T}_{p}
$$

of G-equivariant vector bundles over $\mathrm{X}^{\prime}$, and therefore a canonical isomorphism

$$
A d\left(\mathrm{X}^{\prime}\right) \simeq f^{*} A d(\mathrm{X})
$$

of vector bundles over $\mathrm{B}^{\prime}$. Any connection $\mathrm{H}$ on $\mathrm{X}$ defines by pull-back a connection on $\mathrm{X}^{\prime}$, namely the unique connection $\mathrm{H}^{\prime}$ whose connection form $\theta_{\mathrm{H}^{\prime}}$ is the pull-back by $\mathrm{F}$ of $\theta_{\mathrm{H}}$, i.e., the image of the section $\mathrm{F}^{*} \theta_{\mathrm{H}}$ of $\mathrm{F}^{*}\left(\Omega_{\mathrm{X}}^{1} \otimes \mathrm{T}_{p}\right) \simeq \mathrm{F}^{*}\left(\Omega_{\mathrm{X}}^{1}\right) \otimes \mathrm{F}^{*}\left(\mathrm{~T}_{p}\right)$ by the tensor product of the differential of $\mathrm{F}$, seen as a morphism $\mathrm{F}^{*}\left(\Omega_{\mathrm{X}}^{1}\right) \rightarrow \Omega_{\mathrm{X}^{\prime}}^{1}$ and of the 
isomorphism (A.2). The curvature of $\mathrm{H}^{\prime}$ is the pull-back by $f$ of the curvature of $\mathrm{H}$. (This pull-back is again well-defined, thanks to the isomorphism (A.3).) In particular, if $\mathrm{H}$ is flat, then $\mathrm{H}^{\prime}$ is flat. In this case, when moreover $\mathrm{K}$ is a field of characteristic $p>0$, the $p$-curvature of $\mathrm{H}^{\prime}$ is the pull-back by $f$ of the $p$-curvature of $\mathrm{H}$ (compare with [Kat82], Appendix).

Change of structural group. - If $\mathrm{G}^{\prime}$ is another smooth algebraic group over $\mathrm{K}$ and if $\varphi: G \rightarrow G^{\prime}$ is a morphism of algebraic groups over $K$, then we may associate a principal $G^{\prime}$-bundle over $B$ to any principal $G$-bundle $X$ over $B$ by forming the quotient ${ }^{\varphi} \mathrm{X}:=\mathrm{G} \backslash\left(\mathrm{X} \times{ }_{\mathrm{K}} \mathrm{G}^{\prime}\right)$ of $\mathrm{X} \times{ }_{\mathrm{K}} \mathrm{G}^{\prime}$ by the left-action of $\mathrm{G}$ defined by:

$$
g .\left(x, g^{\prime}\right):=\left(x . g^{-1}, \varphi(g) g^{\prime}\right) \text {. }
$$

This quotient is a well-defined smooth $\mathrm{K}$-scheme by an elementary argument of étale descent (see for instance [Ser58], 3.23$)$ ). The right-action of $\mathrm{G}^{\prime}$ on $\mathrm{X} \times{ }_{\mathrm{K}} \mathrm{G}^{\prime}$ and the morphism $p \circ p r_{1}: \mathrm{X} \times_{\mathrm{K}} \mathrm{G}^{\prime} \rightarrow \mathrm{B}$ induce a right-action of $\mathrm{G}^{\prime}$ on ${ }^{\varphi} \mathrm{X}$ and a smooth morphism ${ }^{\varphi} p:{ }^{\varphi} \mathrm{X} \rightarrow \mathrm{B}$, which make ${ }^{\varphi} \mathrm{X}$ a principal $\mathrm{G}^{\prime}$-bundle over $\mathrm{B}$. There exists a unique morphism $\Phi: \mathrm{X} \rightarrow{ }^{\varphi} \mathrm{X}$ of $\mathrm{B}$-schemes which map a geometric point $x$ of $\mathrm{X}$ to the class $[(x, e)]$ in ${ }^{\varphi} \mathrm{X}$. It is equivariant - namely, it satisfies $\Phi(x . g)=\Phi(x) \cdot \varphi(g)-$ and, for any connection $\mathrm{H}$ on the principal G-bundle $\mathrm{X}$, there exists a unique connection ${ }^{\varphi} \mathrm{H}$ on the principal $\mathrm{G}^{\prime}$-bundle ${ }^{\varphi} \mathrm{X}$ such that the differential of $\Phi$ maps $\mathrm{H}$ onto ${ }^{\varphi} \mathrm{H}$. Moreover, the curvature of ${ }^{\varphi} \mathrm{H}$ is the image of the one of $\mathrm{H}$ by the morphism

$$
\Omega_{\mathrm{B}}^{2} \otimes A d(\mathrm{X}) \longrightarrow \Omega_{\mathrm{B}}^{2} \otimes A d\left(\mathrm{X}^{\prime}\right)
$$

deduced from the morphism of vector bundles over B

$$
A d(\mathrm{X}) \longrightarrow \operatorname{Ad}\left(\mathrm{X}^{\prime}\right)
$$

defined by the linear map

$$
\text { Lie } \varphi: \text { Lie } G \longrightarrow \text { Lie } G^{\prime} \text {. }
$$

In particular, if $\mathrm{H}$ is flat, then ${ }^{\varphi} \mathrm{H}$ is flat. In this case, when moreover $\mathrm{K}$ is a field of characteristic $p>0$, the $p$-curvature of ${ }^{\varphi} \mathrm{H}$ is the image of the $p$-curvature of $\mathrm{H}$ by the morphism

$$
\operatorname{Frob}^{*} \Omega_{\mathrm{B}}^{1} \otimes \operatorname{Ad}(\mathrm{X}) \longrightarrow \operatorname{Frob}^{*} \Omega_{\mathrm{B}}^{1} \otimes \operatorname{Ad}\left(\mathrm{X}^{\prime}\right)
$$

deduced from (A.4).

Quotient by a finite group. - Suppose now that X and B are equipped with leftactions of some finite group $\mathfrak{G}$, that the morphism $p: \mathrm{X} \rightarrow \mathrm{B}$ is $\mathfrak{G}$-equivariant, and that the actions of $\mathfrak{G}$ and $\mathrm{G}$ on $\mathrm{X}$ commute (namely, for any $\gamma \in \mathfrak{G}$ and any geometric points $x$ in $\mathrm{X}$ and $g$ in $\mathrm{G}, \gamma \cdot(x \cdot g)=(\gamma \cdot x) \cdot g)$. Suppose also that $\mathrm{X}$ and $\mathrm{B}$ are 
quasi-projective K-schemes - the quotients $\mathfrak{G} \backslash \mathrm{X}$ and $\mathfrak{G} \backslash \mathrm{B}$ are therefore well-defined (quasi-projective) $\mathrm{K}$-schemes - and that the quotient $\widetilde{\mathrm{B}}:=\mathfrak{G} \backslash \mathrm{B}$ is smooth ${ }^{(15)}$ over $\mathrm{K}$. Let $\widetilde{\mathrm{X}}$ be the quotient $\mathfrak{G} \backslash \mathrm{X}$, and consider the morphism $\widetilde{p}: \widetilde{\mathrm{X}} \rightarrow \widetilde{\mathrm{B}}$ and the rightaction of $\mathrm{G}$ on $\widetilde{\mathrm{X}}$ induced by $p$ and the right-action of $\mathrm{G}$ on $\mathrm{X}$. Then $\widetilde{\mathrm{X}}$ is a principal G-bundle over $\widetilde{\mathrm{B}}$ provided the following condition is satisfied: every geometric point of $\mathrm{B}$ possesses a $\mathfrak{G}$-invariant étale neighbourhood on which the $\mathrm{G}$-bundle $\mathrm{X}$ admits a $\mathfrak{G}$-invariant section.

When this holds, the G-bundle $\mathrm{X}$ over $\mathrm{B}$ is canonically isomorphic to the inverse image of the G-bundle $\widetilde{\mathrm{X}}$ over $\widetilde{\mathrm{B}}$ by the quotient map $f: \mathrm{X} \rightarrow \widetilde{\mathrm{X}}$. If moreover $\mathrm{K}$ is a field of characteristic 0 , any $\mathfrak{G}$-invariant connection $\mathrm{H}$ on $\mathrm{X}$ "descends" to a connection $\widetilde{\mathrm{H}}$ on $\widetilde{\mathrm{X}}$. (In other words, there exists a unique connection $\widetilde{\mathrm{H}}$ on $\widetilde{\mathrm{X}}$ whose inverse image by $f$ coincides with $\mathrm{H}$.)

\section{REFERENCES}

[Anc90] A. Ancona, Théorie du potentiel sur les graphes et les variétés, in École d'été de Probabilités de Saint-Flour XVIII-1988, Lectures Notes in Mathematics 1427, pages 1-112, Berlin, Springer, 1990.

[And89] Y. ANDRé, G-functions and geometry, Braunschweig, Friedr. Vieweg \& Sohn, 1989.

[And99] Y. André, Sur la conjecture des p-courbures de Grothendieck et Katz, Preprint, Institut de Mathématiques de Jussieu, 1999.

[AS60] L. V. Ahlfors and L. Sario, Riemann surfaces, Princeton, N.J., Princeton University Press, 1960, Princeton Mathematical Series, No. 26.

[BMQ01] F. A. Bogomolov and M. L. McQuillan, Rational curves on foliated varieties, Preprint, IHES, 2001.

[Bom81] E. Bombieri, On G-functions, in Recent progress in analytic number theory, Vol. 2 (Durham, 1979), pages 1-67. London, Academic Press, 1981.

[Bos96] J.-B. Bost, Périodes et isogénies des variétés abéliennes sur les corps de nombres (d'après D. Masser et G. Wüstholz), Séminaire Bourbaki, 1994/1995, Exposé No 795, Astérisque, 237 (1996), 115-161.

[Bos98] J.-B. Bost, $\widehat{\mu}_{\max }\left(\mathrm{S}^{k} \overline{\mathrm{E}}\right) \leqslant k\left[\widehat{\mu}_{\max }(\overline{\mathrm{E}})+\mathrm{C}(\mathrm{rkE})\right]$, Letter to P. Graftieaux, December 1998.

[BGS94] J.-B. Bost, H. Gillet, and C. Soulé, Heights of projective varieties and positive Green forms, F. Amer. Math. Soc., 7 (1994), 903-1027.

[CB01] A. Chambert-Loir, Théorèmes d'algébricité en géométrie diophantienne (d'après J.-B. Bost, Y. André, D. et G. Chudnovsky), Séminaire Bourbaki, Exposé 886, mars 2001.

[CC85a] D. V. Chudnovsky and G. V. Chudnovsky, Applications of Padé approximations to the Grothendieck conjecture on linear differential equations, in Number theory (New York, 1983-84), Lectures Notes in Mathematics 1135, pages 52-100, Berlin, Springer, 1985.

[CG85b] D. V. Ghudnovsky and G. V. Chudnovsky, Padé approximations and Diophantine geometry, Proc. Nat. Acad. Sci. U.S.A., 82 (1985), 2212-2216.

[DGS94] B. Dwork, G. Gerotto, and F. J. Suldrvan, An introduction to G-functions, Princeton, N.J., Princeton University Press, 1994.

[Eke87] T. Ekedahl, Foliations and inseparable morphisms, in Algebraic geometry - Bowdoin 1985, Proc. Symp. Pure Math. 46-2, pages 139-149, Amer. Math. Soc., Providence, RI, 1987.

(15) We need this artificial hypothesis to stay in the "smooth" framework considered in this Appendix. 
[ESB99] T. Ekedahl and N. I Shepherd-Barron, A conjecture on the existence of compact leaves of algebraic foliations, Preprint, April 1999.

[Fal83] G. Fattings, Endlichkeitssätze für abelsche Varietäten über Zahlkörpern, Invent. Math., 73 (1983), 349-366.

[Gr98] P. Graftieaux, Groupes formels et critères d'isogénie, Thèse, Université Paris VI, March 1998.

[Gr01a] P. Graftieaux, Formal groups and the isogeny theorem, Duke Math. f., 106 (2001), 81-121.

[Gr01b] P. Graftieaux, Formal subgroups of abelian varieties, Invent. Math., 145 (2001), 1-17.

[GK73] P. GRIFFITHS and J. KING, Nevanlinna theory and holomorphic mappings between algebraic varieties, Acta Math., 130 (1973), 145-220.

[Gri99] A. Grigor'Yan, Analytic and geometric background of recurrence and non-explosion of the Brownian motion on Riemannian manifolds, Bull. Amer. Math. Soc. (N.S.), 36 (1999), 135-249.

[Gro71] A. Grothendieck, Revêtements Étales et Groupe Fondamental, S.G.A.1, Lecture Notes in Mathematics 224, Berlin, Springer-Verlag, 1971.

[GS92] H. Gillet and C. Soulé, An arithmetic Riemann-Roch theorem, Invent. Math., 110 (1992), 473-543.

[Har68] R. Hartshorne, Cohomological dimension of algebraic varieties, Ann. Math., 88 (1968), 403-450.

[Hir68] H. Hironaka, On some formal imbeddings, Illinois f. Math., 12 (1968), 587-812.

[HM68] H. Hironaka and H. Matsumura, Formal functions and formal embeddings, 7. Math. Soc. Fapan, 20 (1968), 52-82.

[Hon68] T. Honda, Formal groups and zeta-functions, Osaka f. Math., 5 (1968), 199-213.

[Hör94] L. Hörmander, Notions of convexity, Boston, MA, Birkhäuser Boston Inc., 1994.

[Kat70] N. Katz, Nilpotent connections and the monodromy theorem: applications of a result of Turrittin, Publ. Math. IHES, 39 (1970), 175-232.

[Kat72] N. Katz, Algebraic solutions of differential equations (p-curvature and the Hodge filtration), Invent. Math., 18 (1972), 1-118.

[Kat73] N. Katz, Exposé XXII: Une formule de congruence pour la fonction $\zeta$, in Groupes de monodromie en géométrie algébrique II, SGA 7 II, Lecture Notes in Mathematics 340, pages 401-438. Berlin, Springer-Verlag, 1973.

[Kat82] N. Katz, A conjecture in the arithmetic theory of differential equations, Bull. Soc. Math. France, 110 (1982), 203-239 and 347-348.

[KM85] N. Katz and B. Mazur, Arithmetic moduli of elliptic curves, Princeton, N.J., Princeton University Press, 1985.

[Kli91] M. Kurmek, Pluripotential theory, New York, The Clarendon Press - Oxford University Press, 1991.

[KN63] S. Kobayashi and K. Nomizu, Foundations of Differential Geometry, New York, John Wiley \& Sons, 1963.

[Kro80] L. Kronecker, Über die Irreductibilität von Gleichungen, Monatsberichte der Königlich Preussischen Akademie der Wissenschaften zu Berlin, pages 155-162, 1880.

[Miy87] Y. MryaокA, Deformations of a morphism along a foliation and applications, in Algebraic geometry-Bowdoin 1985, Proc. Symp. Pure Math. 46-1, pages 245-268. Amer. Math. Soc., Providence, RI, 1987.

[MP97] Y. Miyaoka and T. Peternell, Geometry of higher-dimensional algebraic varieties, Basel, Birkhäuser Verlag, 1997.

[Mum70] D. Mumford, Abelian varieties, Tata Institute of Fundamental Research Studies in Mathematics, No. 5, Bombay, 1970.

[Ser58] J.-P. Serre, Espaces fibrés algébriques, in Séminaire C. Chevalley, Secrétariat Mathématique, Paris, 1958.

[Ser59] J.-P. Serre, Groupes algébriques et corps de classes, Paris, Herman, 1959.

[Ser68] J.-P. SERre, Abelian l-adic representations and elliptic curves, W. A. Benjamin, Inc., New York-Amsterdam, 1968.

[Sha85] B. V. Shabat, Distribution of values of holomorphic mappings, American Mathematical Society, Providence, RI, 1985.

[SB92] N. Shepherd-barron, Miyaoka's theorems on the generic seminegativity of $\mathrm{T}_{\mathrm{X}}$ and on the Kodaira dimension of minimal regular threefolds, Astérisque, 211 (1992), 103-114. 
[Shi75] B. Shiffman, Nevanlinna defect relations for singular divisors, Invent. Math., 31 (1975), 155-182.

[Sie29] C. L. Siegel, Über einige Anwendungen diophantischer Approximationen, Abh. Preuss. Akad. Wiss., 1, 1929.

[Sou97] G. Soulé, Hermitian vector bundles on arithmetic varieties, in Algebraic geometry-Santa Cruz 1995, Proc. Symp. Pure Math. 62-1, pages 383-419, Amer. Math. Soc., Providence, RI, 1997.

[Sto77] W. Stoll, Aspects of value distribution theory in several complex variables, Bull. Amer. Math. Soc., 83 (1977), 166-183.

[Szp85] L. SzPrRo, Degrés, intersections, hauteurs, Astérisque, 127 (1985), 11-28.

[Tak93] K. TAkegoshi, A Liouville theorem on an analytic space, 7. Math. Soc. Fapan, 45 (1993), 301-311.

[Zha98] S.-W. Zhang, Small points and Arakelov theory, in Proceedings of the International Congress of Mathematicians, Vol. II (Berlin, 1998), pages 217-225, 1998.

\section{J.-B. B.}

Département de Mathématiques,

Université Paris-Sud,

Bâtiment 425,

91405 Orsay cedex, France. 